\title{
A Laser-Source Integrating Sphere Reflectometer
}

A Report to: Air Force Materials Laboratory

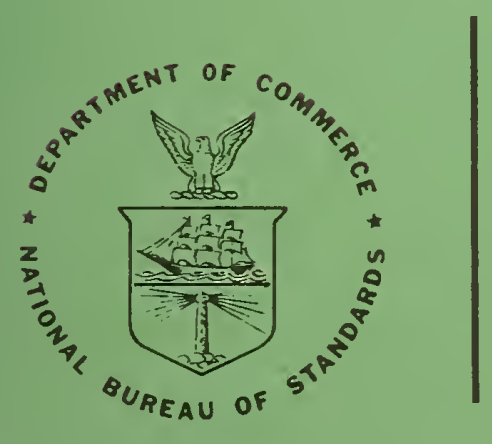

U.S. DEPARTMENT OF COMMERCE National Bureau of Standards 


\section{THE NATIONAL BUREAU OF STANDARDS}

The National Bureau of Standards ${ }^{1}$ provides measurement and technical information services essential to the efficiency and effectiveness of the work of the Nation's scientists and engineers. The Bureau serves also as a focal point in the Federal Government for assuring maximum application of the physical and engineering sciences to the advancement of technology in industry and commerce. To accomplish this mission, the Bureau is organized into three institutes covering broad program areas of research and services:

THE INSTITUTE FOR BASIC STANDARDS ... provides the central basis within the United States for a complete and consistent system of physical measurements, coordinates that system with the measurement systems of other nations, and furnishes essential services leading to accurate and uniform physical measurements throughout the Nation's scientific community, industry, and commerce. This Institute comprises a series of divisions, each serving a classical subject matter area:

-Applied Mathematics-Electricity-Metrology-Mechanics-Heat-Atomic Physics-Physical Chemistry-Radiation Physics--Laboratory Astrophysics ${ }^{2}$-Radio Standards Laboratory, ${ }^{2}$ which includes Radio Standards Physics and Radio Standards Engineering-Office of Standard Reference Data.

THE INSTITUTE FOR MATERIALS RESEARCH ... conducts materials research and provides associated materials services including mainly reference materials and data on the properties of materials. Beyond its direct interest to the Nation's scientists and engineers, this Institute yields services which are essential to the advancement of technology in industry and commerce. This Institute is organized primarily by technical fields:

-Analytical Chemistry-Metallurgy-Reactor Radiations-Polymers-Inorganic Materials-Cryogenics ${ }^{2}$-Office of Standard Reference Materials.

THE INSTITUTE FOR APPLIED TECHNOLOGY ... provides technical services to promote the use of available technology and to facilitate technological innovation in industry and government. The principal elements of this Institute are:

-Building Research-Electronic Instrumentation-Technical Analysis-Center for Computer Sciences and Technology-Textile and Apparel Technology Center-Office of Weights and Measures -Office of Engineering Standards Services-Office of Invention and Innovation-Office of Vehicle Systems Research-Clearinghouse for Federal Scientific and Technical Information ${ }^{3}$-Materials Evaluation Laboratory-NBS/GSA Testing Laboratory.

1 Headquarters and Laboratories at Gaithersburg, Maryland, unless otherwise noted; mailing address Washington, D. C., 20234.

2 Located at Boulder, Colorado, 80302.

${ }^{3}$ Located at 5285 Port Royal Road, Springfield, Virginia 22151. 


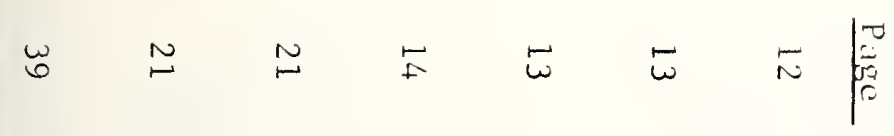

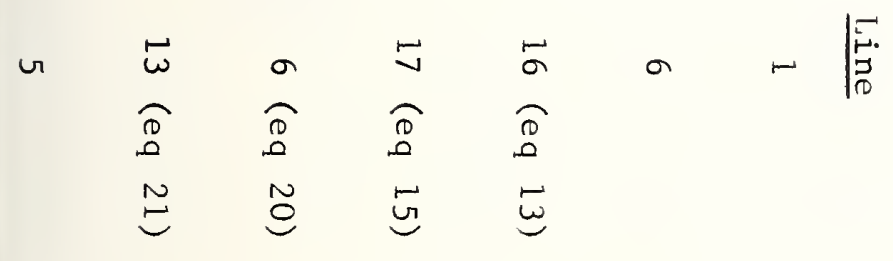

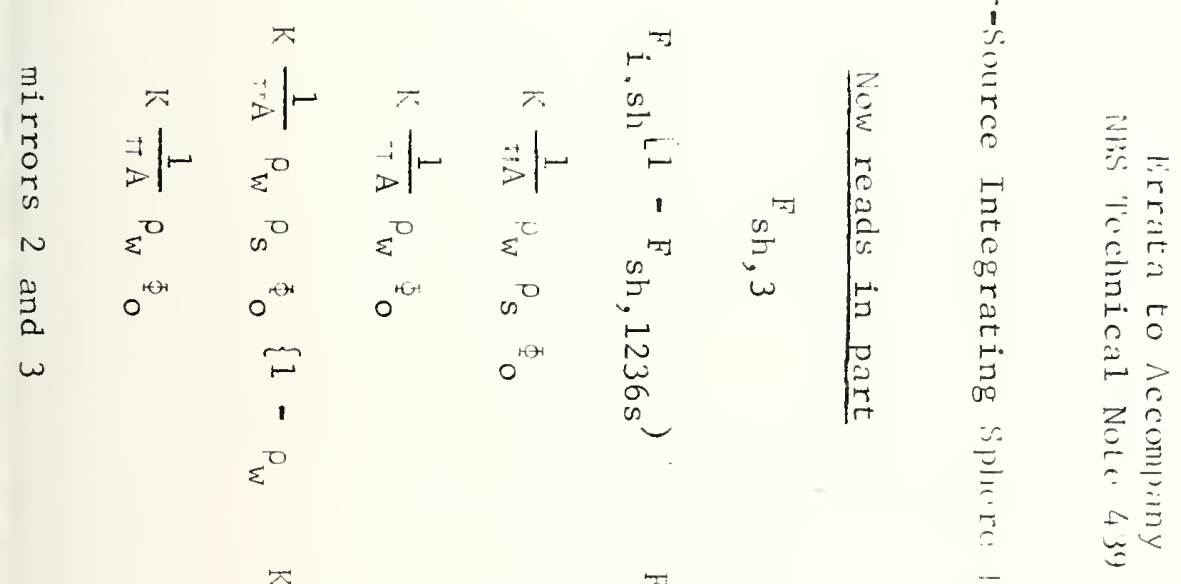

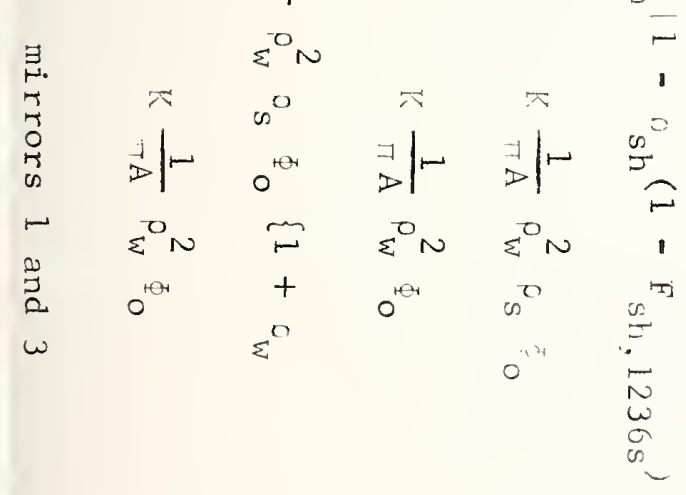





\title{
NBS TECHNICAL NOTE 439 ISSUED FEBRUARY 1968
}

\section{A Laser-Source Integrating Sphere Reflectometer}

\author{
Gerhart J. Kneissl and Joseph C. Richmond
}

Institute for Basic Standards

National Bureau of Standards

Washington, D.C. 20234

\author{
A Report to \\ Air Force Materials Laboratory \\ Research and Technology Division \\ Air Force Systems Command \\ Wright-Patterson Air Force Base, Ohic \\ Project No. 7381, Task No. 738106 \\ (Prepared Under Contract No. (33-615) 65-5005 \\ Amendments No. 2 and 3)
}

\begin{abstract}
NBS Technical Notes are designed to supplement the Bureau's regular publications program. They provide a means for making available scientific data that are of transient or limited interest. Technical Notes may be listed or referred to in the open literature.
\end{abstract}



1. Introduction . . . . . . . . . . . . . . . . . . . . . . . . . 1

1.1. Difficulties of Direct Emittance Measurements . . . . . . . . . . . . . . . 1

1.2. The Reflectance Approach . . . . . . . . . . . . . . . . . . . . 3

2. Description and Theoretical Analysis of the Integrating Sphere . . . . . . . . . . 5

2.1. Basic Theory . . . . . . . . . . . . . . . . . . . . . . 5

2.2. Design of the Integrating Sphere . . . . . . . . . . . . . . . . . . . 6

2.3. Error Analysis of the Integrating Sphere . . . . . . . . . . . . . . . . . . 9

2.3.1. Perfectly Diffuse Sample . . . . . . . . . . . . . . . . . . 9

2.3.2. Perfectly Specular Sample . . . . . . . . . . . . . . . . 16

3. Description of the Experimental Setup . . . . . . . . . . . . . . . . . . . 25

4. Description of the Samples . . . . . . . . . . . . . . . . . . 30

4.1. Thoria . . . . . . . . . . . . . . . . . . . . . . . . 31

4.2. Graphite . . . . . . . . . . . . . . . . . . . . . . 31

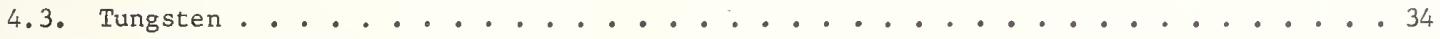

5. Results and Conclusions . . . . . . . . . . . . . . . . . . . . 38

5.1. Experimental Verification of the Error Analysis . . . . . . . . . . . . . . 38

5.1.1. Preparation of the Mirrors . . . . . . . . . . . . . . . 38

5.1.2. Description of the Specular Reflectometer . . . . . . . . . . . . . 38

5.1.3. Calibration of the Mirrors . . . . . . . . . . . . . . . . . 40

5.1.4. Results of Specular Measurements . . . . . . . . . . . . . . . 41

5.1.5. Preparation of Integrating Sphere . . . . . . . . . . . . . . 43

5.1.6. Measurements of the Mirrors in the Integrating Sphere . . . . . . . . 43

5.1.7. Results of the Experimental Evaluation . . . . . . . . . . . . . . . 44

5.2. High Temperature Reflectance Measurements . . . . . . . . . . . . . . . 45

5.2.1. Temperature Measurements . . . . . . . . . . . . . . . . 45

5.2.2. Thoria . . . . . . . . . . . . . . . . . 47

5.2.3. Graphite . . . . . . . . . . . . . . . . . . . . . 49

5.2.4. Tungsten . . . . . . . . . . . . . . . . . 49

6. Conclusions .. . . . . . . . . . . . . . . . . . . . . . . 52

7. References . . . . . . . . . . . . . . . . . . . . . . . . 53

8. Appendix A - The Use of Sodium Chloride as an Integrating Sphere Coating . . . . . . . 54

9. Appendix B - Kirchhoff's Law and the Law of Reciprocity. . . . . . . . . . . . . . 64 
A Laser-Source Integrating Sphere

Reflectometer

by

Gerhart J. Kneissl and

Joseph C. Richmond

\section{Abstract}

A reflectometer was developed for measuring the absolute spectral reflectance of materials at temperatures up to $2500^{\circ} \mathrm{K}$. The equipment included (1) a helium-neon Iaser as the source, capable of operation at $0.6328,1.15$ or $3.39 \mu \mathrm{m}$, (2) a $35 \mathrm{~cm}$ diameter integrating sphe re coated with sodium chloride, (3) a lead sulfide detector, and (4) a radio frequency generator for heating the specimen by induction. A spike filter transmitting at the laser wavelength in front of the detector absorbed most of the background radiation from the hot specimen, and a chopped incident beam together with synchronous amplification of the signal from the detector was used to eliminate the effect of the remaining background radiation. The integrating sphere could be evacuated, or operated under a slight positive pressure of purified helium. An error analysis showed that the measured absolute reflectances are in error by less than one percent. Preliminary data are presented for thoria, tungsten and graphite. Graphite was found to be stable in reflectance on heating to $2150^{\circ} \mathrm{K}$ in vacuum. Both thoria and tungsten were somewhat unstable in reflectance on heating in vacuum.

Key Words: diffuse reflectance, graphite, high temperature reflectance, infrared reflectance, integrating sphere reflectometer, laser, reflectance, sodium chloride coating, synchronous amplification, thoria, tungsten. 


\section{INTRODUCTION}

The knowledge of high temperature themal radiation properties is of great importance for space and military applications. It was the purpose of this research to design, construct and test an instrument which would evaluate the high temperature emittance of refractory metals and ceramics by measuring their reflectance very accurately at high temperatures. The design of heat shields for re-entry vehicles, coatings for rocket nozzles, nosecones and leading edges of airfoils for hypersonic vehicles, to mention only a few, requires the availability of high-temperature emittance data for a wide variety of refractory materials.

The international practical temperature scale above the gold point is defined in terms of radiation and such temperatures are measured mostly by means of an optical pyrometer. The conversion of the brightness temperature of any real material, as measured by the optical pyrometer, to the true temperature requires a knowledge of the emittance of the material at the pyrometer mean effective wavelength. But at the present time, an educated guess is almost as good as any published values for hightemperature emittance, since it is not uncommon for data from different published sources to differ by $100 \%$ for the same material. The reason for this tremendous spread is poor description of the specimen measured and its condition at the time of measurement on the one hand and great experimental difficulties in obtaining high temperature emittance data on the other.

\subsection{Difficulties of Direct Emittance Measurements}

The usual approach of obtaining high temperature thermal radiation properties is to measure spectral emittance directly.

The difficulty of direct emittance measurements arises from definition of emittance as the ratio of flux emitted per unit area from a body to that emitted per unit area from a blackbody (or perfect radiator) at the same temperature, and under the same geometric and spectral conditions.

Emitted flux is a strong function of temperature. To illustrate this point, we can calculate the change of monochromatic flux due to a temperature increase of $1 \%$ as a function of the product $\lambda T$. The monochromatic flux of a blackbody, $\Phi_{\lambda, b}$, defined as the flux emitted per unit area and unit wavelength interval, is calculated from Planck's equation,

$$
{ }^{\Phi} \lambda, \mathrm{b}=\frac{\mathrm{c}_{1}}{\lambda^{5}\left[\left(\exp c_{2} / \lambda \mathrm{T}\right)-1\right]} \text {, }
$$

where $c_{2}$ and $c_{1}$ are constants,

$T$ is the absolute temperature and

$\lambda$ is the wavelength.

Expanding $\Phi_{\lambda, b}$ in a Taylor series and neglecting second and higher order terms yields

$$
\Delta \Phi_{\lambda, \mathrm{b}}=\frac{\partial \Phi_{\lambda, \mathrm{b}}}{\partial \mathrm{T}} \Delta \mathrm{T}
$$

The partial derivative, obtained from equation 1 , is

$$
\frac{\partial \Phi_{\lambda, b}}{\partial T}=\frac{c_{1}}{\lambda^{5}} \frac{\exp c_{2} / \lambda T}{\left[\left(\exp c_{2} / \lambda T\right)-1\right]^{2}} \frac{c_{2}}{\lambda T^{2}} .
$$

Substituting equation 3 into equation 2 and dividing by $\bar{\phi}_{\lambda, \text { b }}$ yields

$$
\frac{\Delta \Phi_{\lambda, b}}{{ }^{\Phi} \lambda, b}=\frac{\exp c_{2} / \lambda T}{\left[\left(\exp c_{2} / \lambda T\right)-1\right]} \frac{c_{2}}{\lambda T} \frac{\Delta T}{T} \text {. }
$$

Figure 1 shows a plot of this function for $\Delta \mathrm{T} / \mathrm{T}=0.01$ as a function of $\lambda \mathrm{T}$. 
If $\Phi_{\lambda, b}$ in eq. 1 is divided by $T^{5}$, this quotient becomes a function of the product $\lambda \mathrm{T}$.

$$
\frac{{ }^{\Phi} \lambda, \mathrm{b}}{\mathrm{T}^{5}}=\frac{\mathrm{c}_{1}}{(\lambda \mathrm{T})^{5}\left[\left(\exp c_{2} / \lambda \mathrm{T}\right)-1\right]}
$$

This function has a maximum value at $\lambda \mathrm{T}=2900 \mu \mathrm{m}^{\circ} \mathrm{K}$. The function (5) divided by its maximum value is then the relative, dimensionless, blackbody function. The second curve shown in figure 1 is a plot of this function. As can be seen from figure 1, the change in flux increases rapidly at wavelengths shorter than the maximum of the blackbody function. At a wavelength corresponding to $\lambda \mathrm{T}$ of $1000 \mu \mathrm{m}^{\circ} \mathrm{K}$, an increase of temperature of $1 \%$ causes an increase of flux of $14.4 \%$. A situation 1 ike this can occur when the temperature of the specimen is measured by a thermocouple and the output from this thermocouple is used to balance the EMF of an identical thermocouple measuring the temperature of the blackbody. The imbalance of the two EMF's is then used to activate a control unit which brings the blackbody to the temperature of the specimen thermocouple. Assuming that the specimen thermocouple measures a temperature which differs from the true temperature by $1 \%$ then the blackbody would be controlled at a temperature which differs by $1 \%$ from the actual specimen temperature. This then would correspond to the situation described above and shown in Figure 1 . If $\epsilon_{\lambda, m}$ is defined as the measured spectral emittance and $\epsilon_{\lambda, t}$ the true spectral emittance, then the error in percent of the true emittance is given by:

$$
E=\frac{\varepsilon_{\lambda, t}-\varepsilon_{\lambda, m}}{\varepsilon_{\lambda, t}} 100
$$

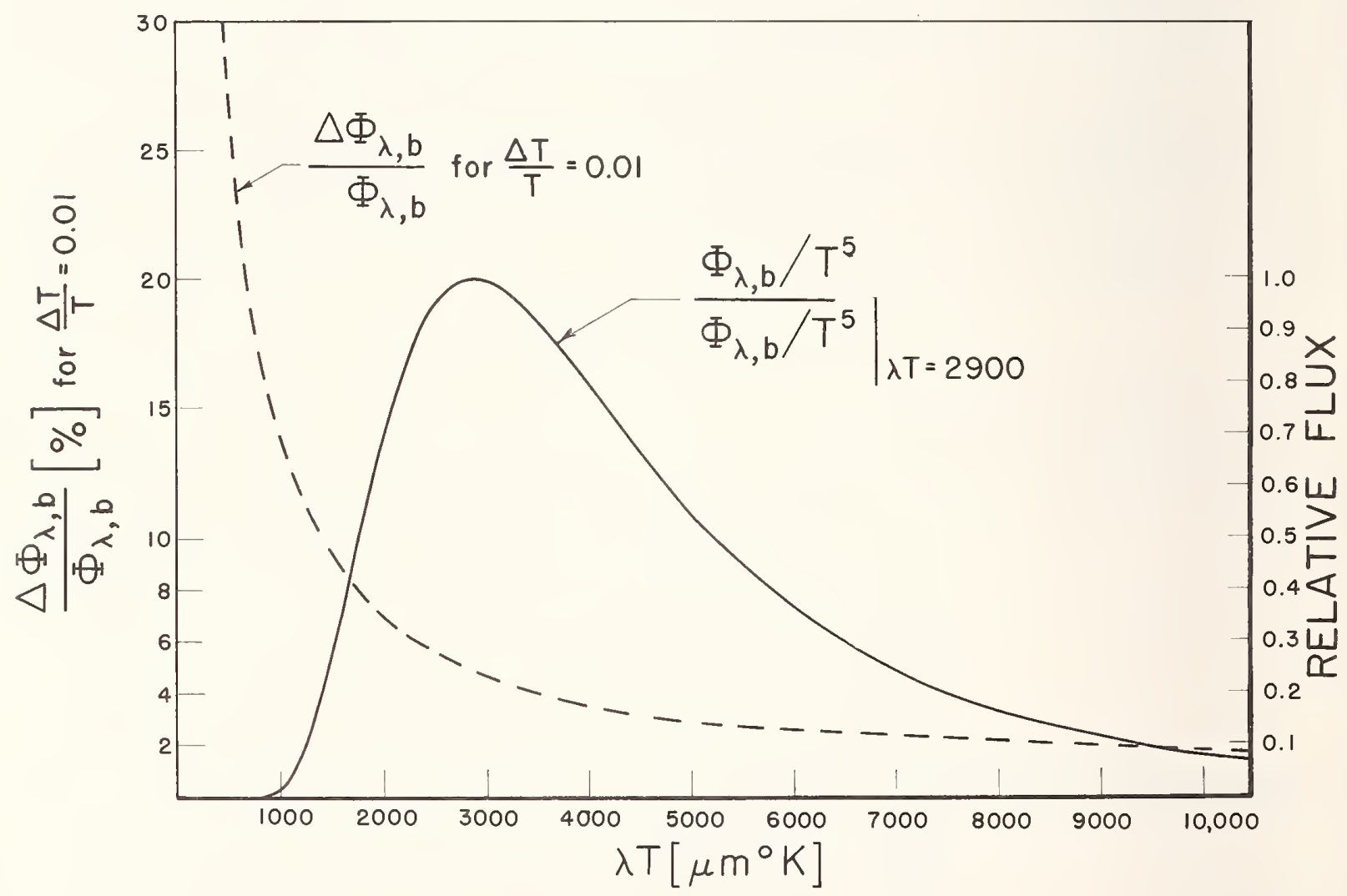

Figure 1. Dimensionless Blackbody Function and Increase in Monochromatic Flux for $1 \%$ Increase of Temperature 


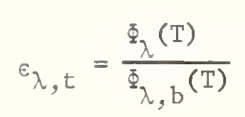

and

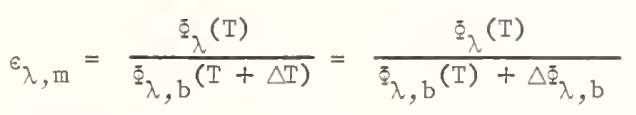

which by the use of equation (7) becomes:

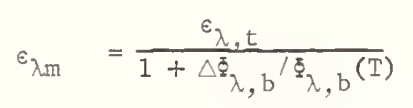

Substituting equation (7) and (9) into equation (6) the error becomes:

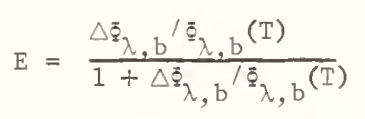

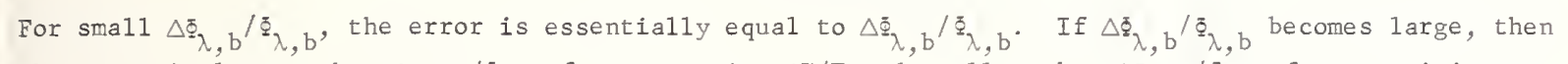

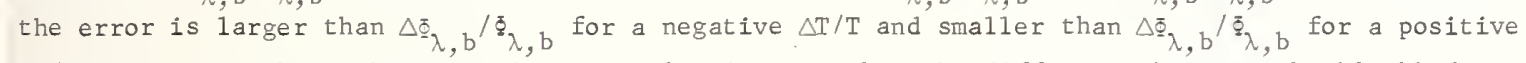
$\Delta T / T$. Figure 2 shows the error in spectral emittance when the difference between the blackbody and the sample is \pm 0.01 of the sample temperature. This shows that very serious errors in spectral emittance can occur due to a temperature difference between sample and reference blackbody.

Even if it is possible to eliminate the temperature difference, it might be almost impossible to measure or even define the temperature of the sample. This is especially true for partially translucent materials of low thermal conductivity. In this case, the emitted flux originates from a finite layer the thickness of which depends on the extinction coefficient, which in turn is a function of wavelength. If the specimen is heated from one side. and radiates from the other, a steep temperature gradient will exist across this effective layer. Theoretically one could define a temperature of an isothermal sample, which would yield the same amount of emitted flux as the real sample with a thermal gradient normal to the surface. However, this effective temperature would be a function of wavelength, since the thickness of the emitting layer is wavelength dependent. In reality this is a hopeless endeavor, due to the lack of knowledge of material properties at the high temperatures involved.

1.2. The Reflectance Approach

All the difficulties of direct emittance measurements can be minimized by measuring the spectral, directional-hemispherical reflectance and calculating the spectral, directional emittance from the equation:

$$
\epsilon_{\lambda}(\theta, \varphi)=1-\rho_{\lambda}(\theta, \varphi ; 2 \tau)
$$

where $\theta, \varphi$ are the same for $\epsilon$ and $\rho$. This equation is derived from Kirchhoff's law and the law of reciprocity (see Appendix $B$ ) and is valid when the transmitted flux is negligible.

In this work, the spectral, directional-hemispherical reflectance was measured by a high accuracy integrating sphere while the sample was being maintained at the desired temperature. Since reflected flux, as opposed to emitted flux, is a very weak function of temperature, the requirement of high accuracy temperature measurements is eliminated.

The principal problem in measuring reflectance of specimens at high temperatures lies in the fact that the reflected flux must be measured in the presence of the flux emitted by the hot specimen. Special techniques are required to measure a small signal in the presence of a large background. The method employed was to use a high intensity source, chopped incident flux and a synchronous detectoramplifier combination. 


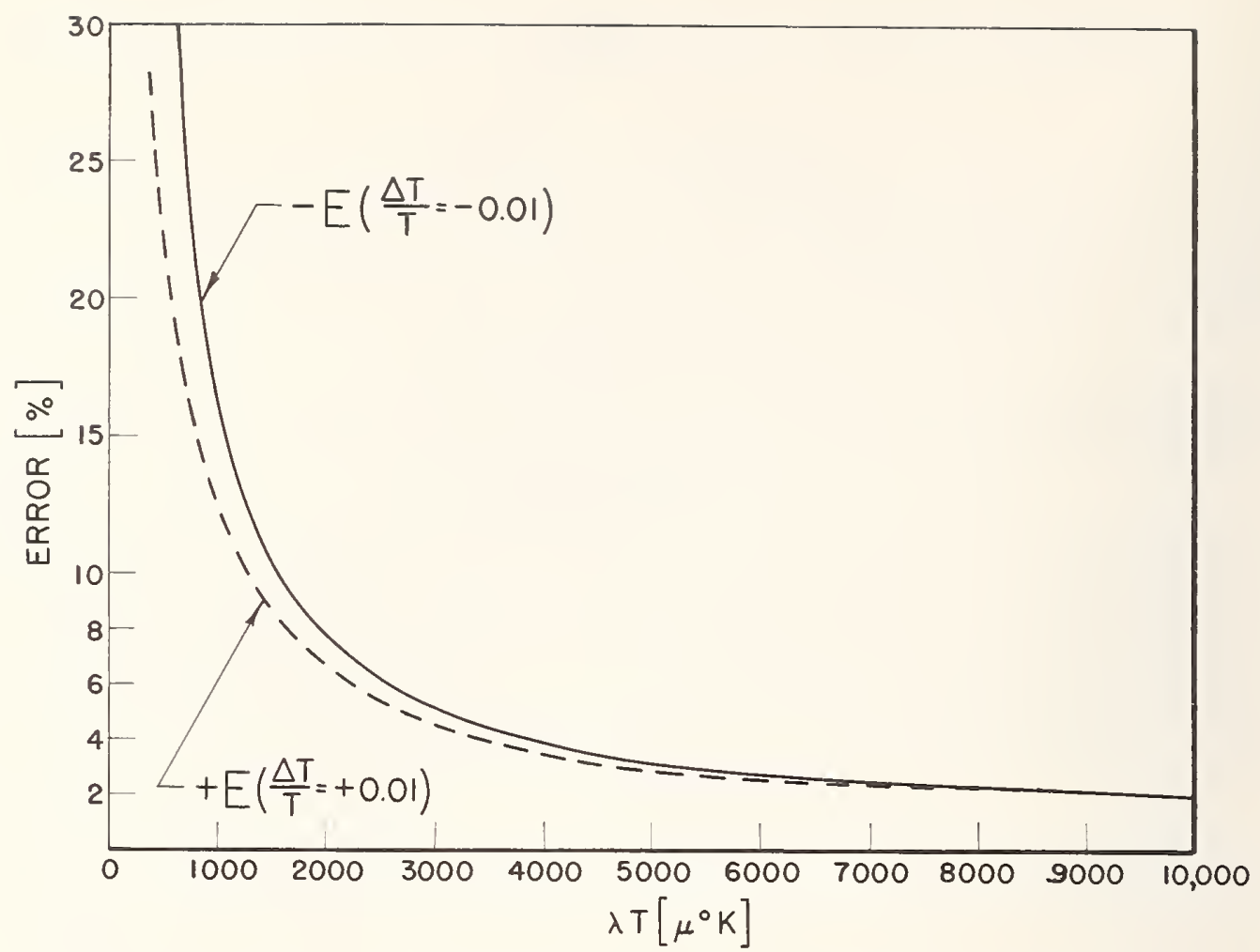

Figure 2. Error in Spectral Emittance Due to a Temperature Difference of $1 \%$ Between Sample and Blackbody.

In order to further increase the signal to noise ratio, a narrow bandpass filter, whose peak transmittance coincided with the particulax source wavelength, was employed in front of the detector. In this way most of the emitted flux was blocked, and only the small portion contained in the transmittance band of the filter could reach the detector. As will be shown in the following sections, this arrangement permitted reflectance measurements of high accuracy and precision regardless of the material measured and its surface conditions. 


\section{DESCRIPTION AND THEORETICAL ANALYSIS OF THE INTEGRATING SPHERE}

The accuracy of the measurements is mainly detemined by the performance of the integrating sphere used in the experiment. This section is, therefore, concerned with the description and theoretical analysis of the integrating sphere as used in the experiment which, based on certain assumptions, will permit limits to be put on the errors involved in the reflectance measurements.

\section{1. Basic Theory}

The theory of the integrating sphere is based on two fundamental laws of radiation.

a. The flux received by an elemental area from a point source is inversely proportional to the square of the distance from the source to the receiving area and directly proportional to the cosine of the angle between the normal to the receiving area and the direction of incidence.

b. The flux reflected by a perfect diffuser follows the cosine distribution law, which means that the flux per unit solid angle reflected from a unit surface area in any given direction is proportional to the cosine of the angle between the normal to the surface and the given direction.

When these laws are applied to a sphere having a perfectly diffuse wall of uniform reflectance, it is found that the flux reflected by an area on the sphere irradiates the sphere wall uniformly. Each elemental surface of unit area on the sphere receives the same amount of radiant flux by reflection. In Figure 3, let $\mathrm{dA}_{1}$ be the reflecting elemental area on the sphere wall and $\mathrm{dA}_{2}$ be the receiving area. These two elemental areas are considered to be so small that they can be treated as plane.

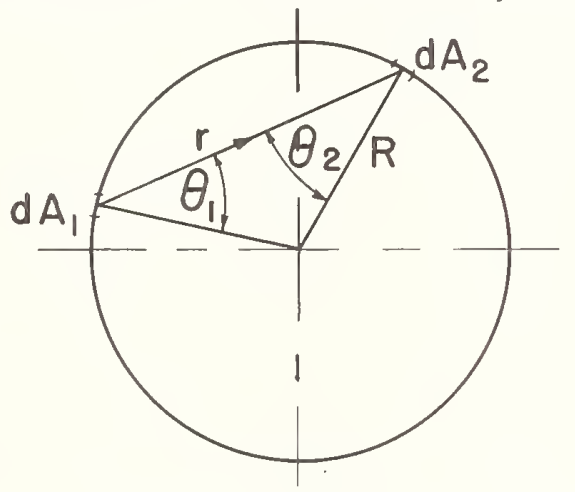

Figure 3. Basic Sphere Geometry

Then the increment of flux $d^{2} \Phi$ that leaves $d_{1}$ and falls directly on $\mathrm{dA}_{2}$ is

$$
\mathrm{d}^{2} \Phi=\mathrm{LdA}_{1} \cos \theta_{1} \mathrm{~d} \omega,
$$

where $L$ is the radiance of $d_{1}$ and $d w$ is the elemental solid angle subtended by $d A_{2}$ at $d A_{1}$.

Since

$$
\begin{aligned}
\mathrm{d} \omega & =\frac{\mathrm{dA}_{2} \cos \theta_{2}}{r^{2}}, \\
\mathrm{~d}^{2} \Phi & =\frac{\mathrm{LdA}_{1} \mathrm{dA}_{2} \cos \theta_{1} \cos \theta_{2}}{\mathrm{r}^{2}},
\end{aligned}
$$

where $r$ is the distance from $\mathrm{dA}_{1}$ to $\mathrm{dA}_{2}$. From Figure 3 we see that

$$
\begin{aligned}
& \theta_{1}=\theta_{2}=\theta, \\
& r=(2 R \cos \theta),
\end{aligned}
$$


and

$$
d^{2} \Phi=\frac{\mathrm{LdA}_{1} \mathrm{dA}_{2} \cos ^{2} \theta}{4 \mathrm{R}^{2} \cos ^{2} \theta}=\frac{\mathrm{LdA}_{1} \mathrm{dA}_{2}}{4 \mathrm{R}^{2}} .
$$

For a perfect diffuser the total reflected flux is dA $\pi \mathrm{L}$; and, therefore, the fraction of the flux incident on $\mathrm{dA}_{2}$ is:

$$
\frac{\mathrm{d}^{2} \mathrm{z}}{\pi \mathrm{LdA}}=\frac{\mathrm{dA}_{2}}{4 \mathrm{R}^{2} \pi}=\frac{\mathrm{dA}_{2}}{\mathrm{~A}}
$$

where $A=4 \pi R^{2}$ is the total area of the sphere. Therefore, in a sphere whose surface reflects in a perfectly diffuse manner, the irradiance due to reflected flux is equal at all points, regardless of the geometric or areal distribution of the incident flux.

\subsection{Design of the Integrating Sphere}

The sphere which was to be used in the laser integrating sphere reflectometer had to fulfill the following requirements.

a. The reflectometer should yield accurate results regardless of directional distribution of the reflected flux from the sample.

b. It should be possible to measure absolute reflectance, as well as reflectance relative to a reference standard.

c. The specimen heater should not disturb the sphere configuration and should be capable of heating the sample to temperatures from room temperature to $2500^{\circ} \mathrm{K}$.

d. The sphere should be capable of operation in vacuum, air, or inert atmosphere.

Before the integrating sphere can be discussed, it is first necessary to describe its geometry. The sphere, as shown in Figure 4, is made up of two hemispheres which are joined by bolted flanges with an 0-ring seal. The principal axis of the sphere is defined as the diameter normal to the plane through the joint connecting the hemispheres. The primary plane is defined as the plane containing the principal axis and the center of the entrance port, and the secondary plane of the sphere is the plane containing the principal axis and normal to the primary plane. The entrance port is centered $12^{\circ}$ from

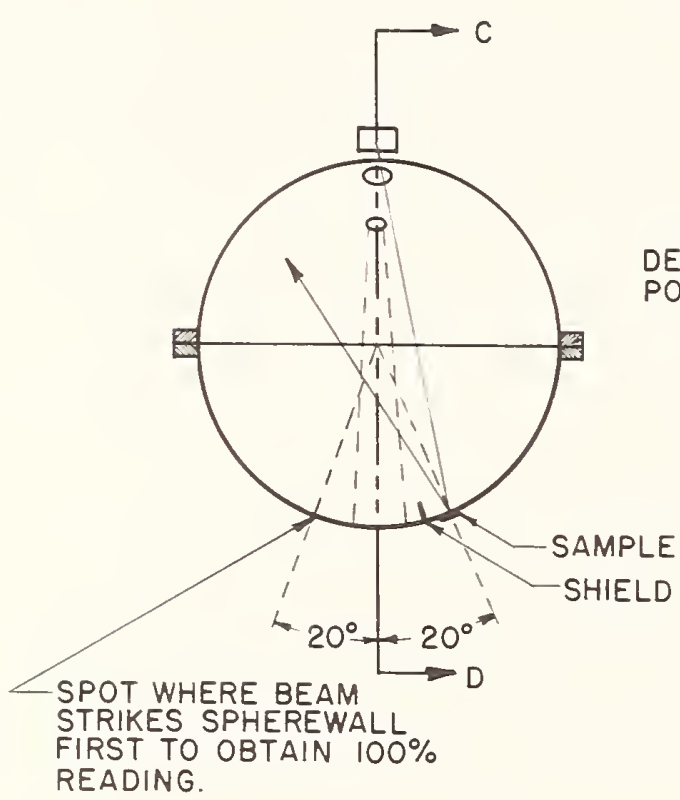

VIEW $A-B$

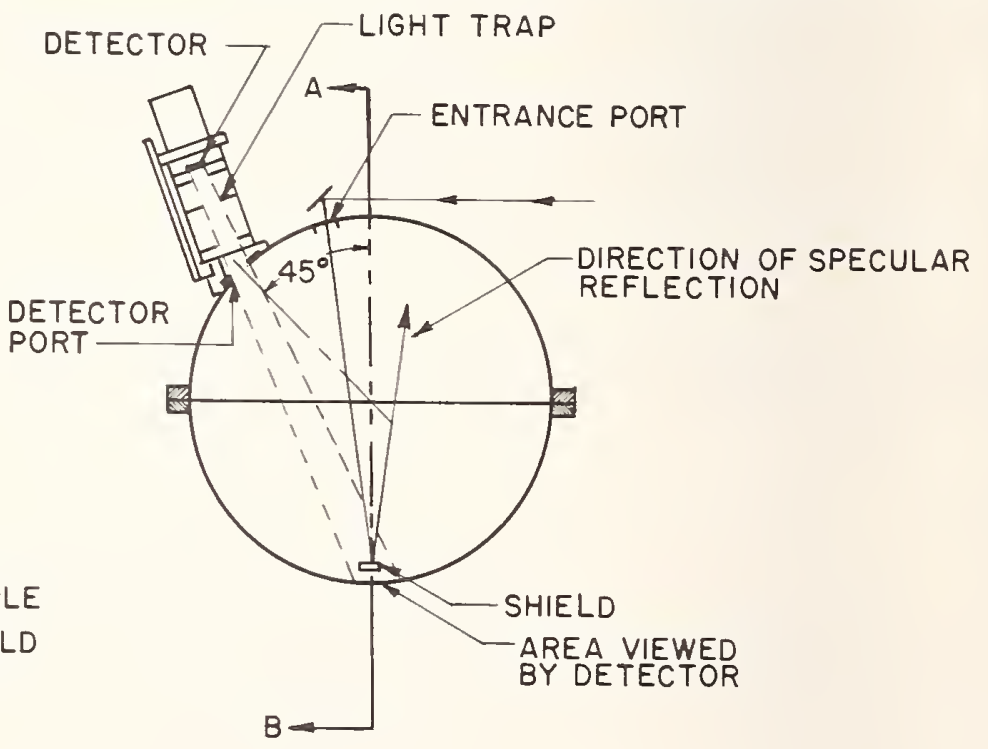

VIEW $\mathrm{C} \longrightarrow \mathrm{D}$

Figure 4. Geometry of the Integrating Sphere 
the principal axis, and by definition is in the primary plane. The detector port is centered $45^{\circ}$ from the principal axis and is also located in the primary plane. The field of view of the detector is restricted to a small area of the sphere wall centered around the principal axis in the lower hemisphere. The ports for the specimen and comparison standard are placed in the secondary plane, $20^{\circ}$ on either side of the principal axis. They are thus located symmetrically with respect to the entrance and detector ports. Small shields screen the specimen and comparison standard ports from the area viewed by the detector.

A sphere designed as described above will meet the requirements established above as indicated in the following paragraphs.

a. Since the field of view of the detector is restricted to a small portion of the sphere wall which is shielded from the sample, no flux reflected from the sample can reach the field of view directly. Thus the reflected flux will be diffused by the sphere coating before it is incident on the area viewed by the detector. Assuming that the sphere coating reflects uniformly in a perfectly diffuse manner, then the flux received at the field of view is always a constant fraction of the flux reflected by the sample, regardless of its directional distribution. The possibility that flux reflected by the sample is received by the detector directly is eliminated by equipping the detector port with a light trap so that any radiation that reaches the detector port from directions outside the field of view is absorbed before reaching the detector.

The field of view of the detector is located symmetrically with respect to sample and comparison standard. When the sphere is used in the comparison mode, the reflected flux from the heated specimen will be compared to that of a water-cooled comparison standard made of the same material and fabricated in the same manner. Therefore, we can assume within reason that the directional distributions of the reflected fluxes are similar. If we assume, furthermore, that the directional distribution will not change with increasing surface temperature, then we can drop the requirement that the sphere coating be a perfect diffuser, and require only that the coating possess uniform reflectance. We can see this in the following manner. Since sample and standard are located symmetrically with respect to the entrance port and field of view, the reflected fluxes will be distributed symmetrically with respect to the field of view as will be the flux after being reflected by the sphere coating. As a consequence, the same fraction of flux leaving the sphere wall will be incident on the field of view in both cases, even if the reflectance function of the coating exhibits a directional variation rather than being a constant.

b. Since it is always desired to obtain an absolute value for reflectance rather than one based on some arbitrary and more or less doubtful standard, it was decided to find a way to use the sphere as an absolute reflectometer. A literature survey revealed that of the many ways possible to achieve this, one method seemed to be especially suitable for the sphere configuration described above. This method has been reported by McNicholas [1] among others, and can be summarized as follows. Assume a sphere configuration as described above where the comparison standard is now replaced by a spherical cap of the same curvature as the sphere and coated in the same manner as the sphere. If the beam is first incident upon the sample shielded from the field of view of the detector, and then upon a portion of the sphere wall not shielded from the detector's field of view, then the ratio of the resulting radiance (detector response) when the sample is irradiated, to the radiance (detector response) when the sphere wall is irradiated, is equal to the absolute directional-hemispherical reflectance of the sample.

The basic possibility of obtaining the absolute value of reflectance, regardless of the directiona1 distribution of the reflected flux, by this method can be proved as follows, if certain assumptions are made. Let $\oint_{0}$ be the flux initially entering the sphere, $\mathrm{A}_{\mathrm{v}}$ the area viewed by the detector, $\mathrm{A}$ the total sphere area, $\rho_{S}(\theta, \varphi ; 2 \pi)$ the directional-hemispherical reflectance of the sample, $p_{W}$ the reflectance of the sphere wall and $L^{\prime}\left(\theta^{\prime}, \varphi^{\prime}\right)$ the radiance of the sample in the direction $\left(\theta^{\prime}, \varphi^{\prime}\right)$, due 
to reflected flux.

The flux $\bar{\Phi}_{0}$ is incident upon the sample from the direction $(\theta, \varphi)$. The flux intercepted by a small area $\mathrm{dA}$ of the sphere in the direction $\left(\theta^{\prime}, \varphi^{\prime}\right)$ as seen from the sample is

$$
L^{\prime}\left(\theta^{\prime}, \varphi^{\prime}\right) A_{i} \cos \theta^{\prime} \mathrm{d} \omega^{\prime},
$$

where $\mathrm{d} \omega^{\prime}$ is the elemental solid angle subtended by $\mathrm{dA}$ at the sample and $\mathrm{A}_{i}$ is the irradiated portion of the sample area.

The flux that leaves dA is

$$
P_{w} L^{\prime}\left(\theta^{\prime}, \varphi^{\prime}\right) A_{i} \cos \theta^{\prime} d \omega^{\prime} .
$$

Since we assume the sphere coating to be a perfectly diffuse reflector the fraction of this flux that reaches the area $A_{v}$ is equal to the configuration factor $F_{d A-A}$ as defined in ref. [9] and [10]. In the case of the sphere this factor has been shown to be $\mathrm{A}_{\mathrm{V}} / \mathrm{A}$.

Thus the irradiance of $\mathrm{A}_{\mathrm{v}}$ due to the flux incident on $\mathrm{dA}$ is

$$
\frac{1}{A} \rho_{W} L^{\prime}\left(\theta^{\prime}, \varphi^{\prime}\right) A_{i} \cos \theta^{\prime} d \omega^{\prime}
$$

As shown by Taylor [2] and many others, the irradiance is increased by the factor $1 /\left(1-\rho_{W}\right)$ due to multiple reflections within the sphere. Therefore, the irradiance of $A_{V}$ that results from reflected flux incident on $\mathrm{dA}$ is

$$
\frac{\rho_{W}}{A\left(1-\rho_{W}\right)} L^{\prime}\left(\theta^{\prime}, \varphi^{\prime}\right) A_{i} \cos \theta^{\prime} d \omega^{\prime} .
$$

The total irradiance due to all the reflected flux is

$$
\frac{\rho_{W}}{A\left(1-\rho_{W}\right)} A_{i} \int_{0}^{2 \pi} L^{\prime}\left(\theta^{\prime}, \varphi^{\prime}\right) \cos \theta^{\prime} d \omega^{\prime},
$$

where the integration is performed over the solid angle of $2 \pi$. This gives rise to a radiance leaving $A_{V}$ of

$$
\frac{1}{\pi} \frac{\rho_{W}^{2}}{A\left(1-\rho_{W}\right)} A_{i} \int_{0}^{2 \pi} L^{\prime}\left(\theta^{\prime}, \varphi^{\prime}\right) \cos \theta^{\prime} d \omega^{\prime} .
$$

Since $A_{i} \int^{2 \pi} L^{\prime}\left(\theta^{\prime}, \varphi^{\prime}\right) \cos \theta^{\prime} d \omega^{\prime}$ is equal to the total reflected flux $\Phi^{\prime}$ from the sample, we can also write the ${ }^{\circ}$ radiance of $A_{v}$ to be

$$
\frac{1}{\pi} \frac{\rho_{W}^{2}}{A\left(1-\rho_{W}\right)} \Phi^{\prime}
$$

To effectively evaluate the initial flux $\Phi_{0}$, we direct the beam onto the sphere wall at a point which is not shielded from $A_{v}$. The flux that leaves the sphere wall is $p_{w} \Phi_{0}$. The fraction of this flux

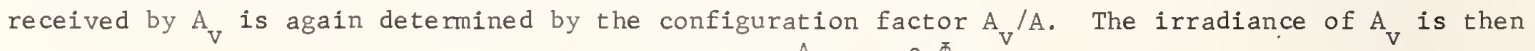
$\frac{1}{A} \rho_{W} \frac{A v}{A}=\frac{\rho_{W}^{\Phi} 0}{A}$.

Again the irradiance is increased by multiple reflections within the sphere by the factor $1 /\left(1\right.$ - $\rho_{W}$ ). Therefore the total irradiance of $A_{V}$ is

$$
\frac{\rho_{W}}{A\left(1-\rho_{W}\right)}{ }^{\Phi_{0}} .
$$

This results in a radiance leaving $A_{v}$ of

$$
\frac{1}{\pi} \frac{\rho_{W}{ }^{2}}{A\left(1-\rho_{W}\right)} \Phi_{0} .
$$

The ratio of the radiances is

$$
\frac{\frac{1}{\pi} \frac{\rho_{W}^{2}}{A\left(1-\rho_{W}\right)} \Phi^{\prime}}{\frac{1}{\pi} \frac{\rho_{W}^{2}}{A\left(1-\rho_{W}\right)} \Phi_{0}^{\Phi_{0}}}=\frac{\Phi^{\prime}}{\Phi_{0}}=\rho_{S}(\theta, \varphi ; 2 \pi),
$$


since $\Phi^{\prime} / \Phi_{0}$ is by definition the directional-hemispherical reflectance of the sample.

It might be useful to restate the assumptions made or implied in the derivation. First, the sphere coating is a perfectly diffuse reflector of uniform reflectance, secondly, the sum of the areas of all openings is negligible compared with the total sphere area, and finally, the presence of the shield does not change measurably the irradiance of the sphere. However, no assumption had to be made as to the directional distribution of $\Phi^{\prime}$.

When the reflectance of the heated specimen was measured, relative to a cooled standard, then the spherical cap was exchanged for a sample holder with a shield attached to it. Thus it is possible to use the sphere in the absolute as well as the relative mode.

c. The method chosen for heating the sample was induction heating. The heating unit, which is described in more detail in Section 3, was mounted outside the sphere and did not disturb the sphere configuration in any way.

d. All ports could be closed by quartz windows with O-ring seals. Also, the heating unit had been built vacuum tight. Slots arranged concentrically around the sample allowed evacuation of the sphere. Inert gas could be introduced through openings in the flange around the entrance port, and exhausted through the evacuation slots and a valve located in the bottom of the heating unit.

\subsection{Error Analysis of the Integrating Sphere}

In the previous section certain simplifying assumptions were made to show the basic capability of obtaining absolute reflectance by the use of an ideal integrating sphere. It is the purpose of this section to find the magnitude of the errors caused by the deviation of a real integrating sphere from the ideal integrating sphere.

Many authors have dealt with the errors involved in the use of an integrating sphere for reflectance measurements. However, none of the published equations can be applied directly to this sphere.

The assumptions under which the following expressions will be derived are that the sphere coating is a perfectly diffuse reflector of uniform reflectance and that all flux that leaves through the openings of the sphere is lost. In addition, we assume that the detector signal is strictly proportional to the radiance of the field of view $A_{v}$. This means that the detector-amplification-recorder system has to be linear over the dynamic range used.

We will consider two cases. First, assume the sample to be a perfectly diffuse reflector. For a perfectly diffuse reflector, the flux intercepted by the shield after its initial reflection from the sample will be a maximum. In the second case, the sample is treated as a perfect mirror. For a perfect mirror no flux reflected once only by the specimen will be incident on the shield, as can be seen from Figure 4. Since the directional distribution of a real sample is somewhere between that of a perfect diffuser and that of a perfect mirror, we will be able to place limits on the magnitude of the error caused by a real sample. (This analysis does not consider retroreflectors, but this is not a severe limitation, since almost all technical materials have a distribution to which the statement above applies.)

\subsubsection{Perfectly Diffuse Sample}

Let $\Phi_{0}$ be the $f l u x$ incident on the sample. Then the flux $\rho_{s} \Phi_{0}$ is reflected diffusely. of this

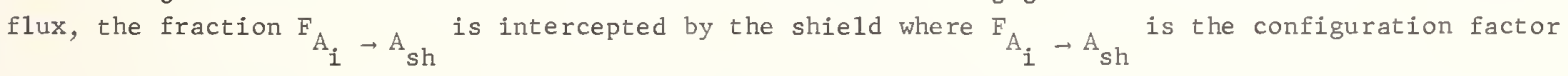
from the irradiated area on the sample to the shield. Figure 5 shows the geometry in its correct proportions. Since the irradiated spot is only about $1 / 8$ inch in diameter, we can use the incremental area approximation for the configuration factor, namely 


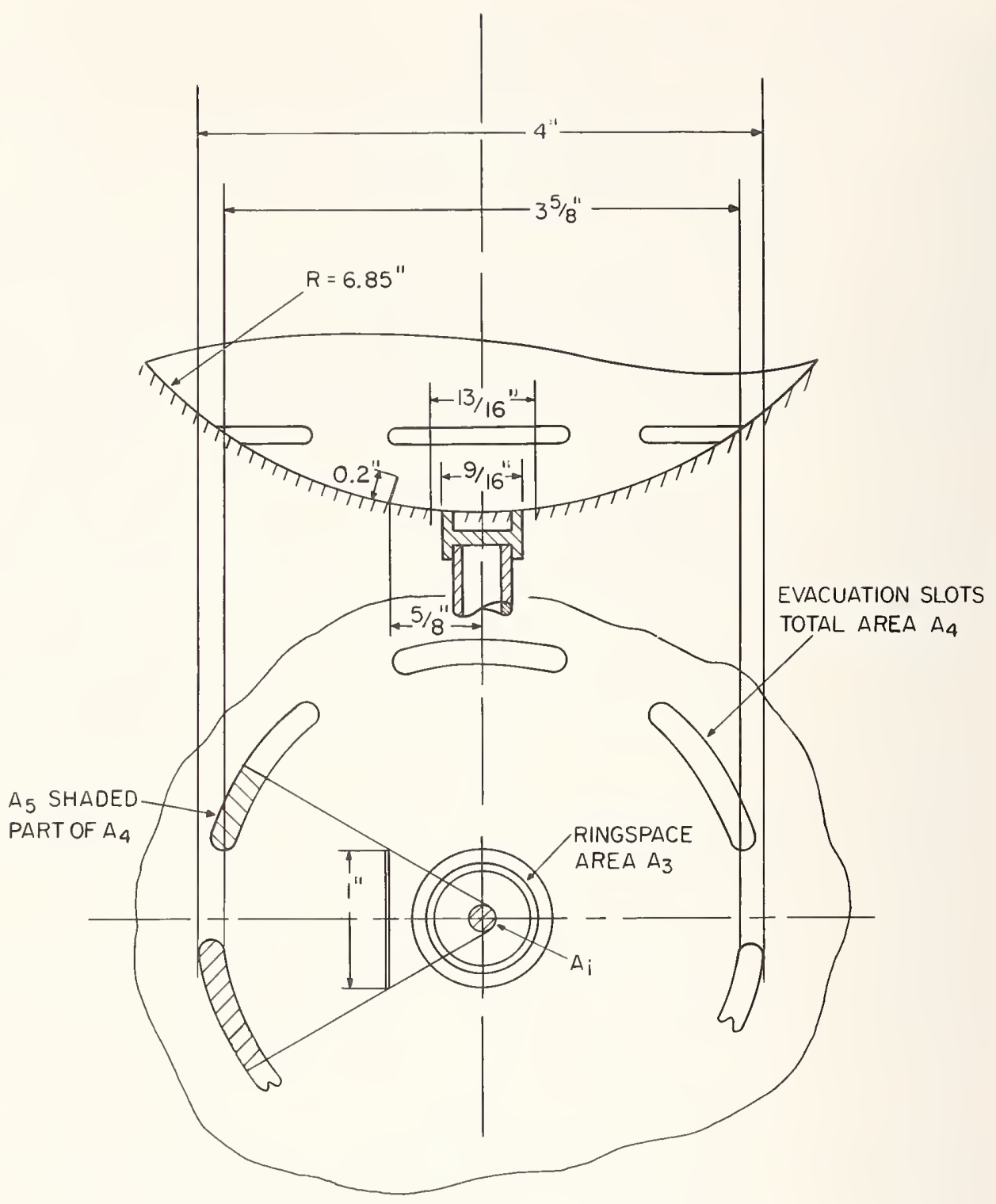

Figure 5. Details of Sample - Shield Geometry

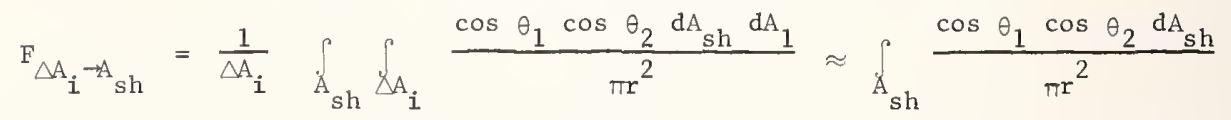

This configuration factor given by Hamilton and Morgan [3] was calculated to be 0.017 for our sphere. At this point, we will adopt an abbreviation for the configuration factors involved. The letter A for area will be omitted and the areas we are dealing with will be designated by their subscripts. For example

$$
\mathrm{F}_{\mathrm{A}_{i} \rightarrow \mathrm{A}_{\mathrm{sh}}}=\mathrm{F}_{i, \mathrm{sh}}
$$

and

$$
\mathrm{F}_{\mathrm{sh}} \rightarrow \mathrm{A}_{1}+\mathrm{A}_{2}+\mathrm{A}_{3}+\mathrm{A}_{4}=\mathrm{F}_{\mathrm{sh}, 1234^{\circ}}
$$

The flux incident on the shield is therefore $\rho_{s} \Phi_{o} F_{i, s h}$. of this flux the fraction $\left(1-\rho_{s h}\right)$ is absorbed, where $P_{s h}$ is the reflectance of the shield. The flux that leaves the shield is

$$
\rho_{\text {Sh }} \rho_{S} \Phi_{0} F_{i, s h}
$$


From Figures 4 and 5 we can see that the shield can view all openings of the sphere with the exception of the shaded part of $A_{4}$ which we name $A_{5}$. The flux lost out the openings is

$$
\rho_{\text {sh }} \rho_{s} \Phi_{0} F_{i, s h}\left(F_{s h, 1}+F_{s h, 2}+F_{s h, 3}+F_{s h, 6}\right)=\rho_{s h} \rho_{s} \Phi_{0} F_{i, s h} F_{s h, 1236}
$$

where the subscript 1 stands for the area of the entrance port,

$$
\begin{aligned}
& 2 \text { stands for the area of the detector port, } \\
& 3 \text { stands for the area of the ring space around the sample, } \\
& 4 \text { stands for the area of the evacuation slots, } \\
& 5 \text { stands for the shaded part of } \mathrm{A}_{4} \text {, }
\end{aligned}
$$

and

$$
6 \text { stands for } A_{6}=A_{4}-A_{5} \text {. }
$$

Another fraction

$$
\rho_{s} \Phi_{0} F_{i, s h} \rho_{s h}{ }^{F} s h, s
$$

of the flux is received by the sample. The remaining flux, which is incident on the sphere wall, is

$$
\rho_{s h} \rho_{s} F_{i, s h}-\rho_{s h} \rho_{s} \Phi_{0} F_{i, s h}\left(F_{s h, 1236}+F_{s h, s}\right)=\rho_{s h} \rho_{s} \Phi_{0} F_{i, s h}\left(1-F_{s h, 1236 s}\right) \text {, }
$$

where the subscript s symbolizes the sample area.

After these preliminary remarks, we can proceed to investigate the diffuse case. The flux $\Phi_{0}$ is incident on the sample and the flux $\rho_{S} \Phi_{0}$ is diffusely reflected by the sample. of the once reflected flux $\rho_{S} \Phi_{0}$, the fraction $F_{i, 126}$ is lost through the openings $A_{1}, A_{2}$, and $A_{6}$ ( $A_{3}$ cannot be "seen" by the sample). The flux incident on the wall directly is therefore

$$
\rho_{s} \Phi_{0}-\rho_{s} \Phi_{0} F_{i, 126}-\rho_{s} \Phi_{0} F_{i, s h}=\rho_{s} \Phi_{0}\left(1-F_{i, 126}-F_{i, s h}\right) \text {. }
$$

In addition to the flux which is incident on the wall directly we have another contribution

$$
\rho_{\text {sh }} \rho_{s} \Phi_{0} F_{i, s h}\left(1-F_{s h, 1236 s}\right)
$$

which, as we have seen, is the portion of the flux reflected by the shield and then incident on the sphere wall.

Al1 the flux incident on the sphere wall after one reflection by the specimen is therefore

$$
\begin{aligned}
\rho_{s} \Phi_{0}(1 & \left.-F_{i, 126}-F_{i, s h}\right)+\rho_{s h} \rho_{s} \Phi_{0} F_{i, s h}\left(1-F_{s h, 1236 s}\right) \\
& =\rho_{s} \delta_{0}\left[1-F_{i, 126}-F_{i, s h}+\rho_{s h} F_{i, s h}\left(1-F_{s h, 1236 s}\right)\right] \\
& =\rho_{s} \Phi_{0}\left\{1-F_{i, 126}-F_{i, s h}\left[1-\rho_{s h}\left(1-F_{s h, 1236 s}\right)\right]\right\} .
\end{aligned}
$$

Up to this point no flux was incident on the detector's field of view $A_{V}$. Only flux reflected by the sphere wall is able to reach $A_{v}$. This flux is

$$
\rho_{W} \rho_{s} \Phi_{0}\left\{1-F_{i, 126}-F_{i, s h}\left[1-\rho_{s h}\left(1-F_{s h, 1236 s}\right)\right]\right\} \text {. }
$$

The flux that strikes $A_{V}$ after one reflection from the sphere wall is

$$
\Phi_{\mathrm{v}, 1}=\frac{\mathrm{A}_{\mathrm{v}}}{\mathrm{A}} \rho_{\mathrm{W}} \rho_{\mathrm{S}} \Phi_{\mathrm{O}}\left\{1-\mathrm{F}_{\mathrm{i}, 126}-\mathrm{F}_{\mathrm{i}, \mathrm{sh}}\left[1-\rho_{\mathrm{sh}}\left(1-\mathrm{F}_{\mathrm{sh}, 1236 \mathrm{~s}}\right)\right]\right\} \text {. }
$$

To evaluate the magnitude of the losses, the configuration factors have been calculated to be

$$
\begin{aligned}
& F_{i, s h}=0.017 \\
& F_{i, 126}=0.0051 \\
& F_{\text {sh,s }}=0.021 \\
& F_{\text {sh, } 1}=0.00069 \\
& F_{\text {sh, }}=0.00026 \\
& F_{\text {sh, } 3}=0.032
\end{aligned}
$$




$$
\begin{aligned}
& F_{\text {sh, }}=0.019 \\
& F_{\text {sh, } 1236 s}=0.073 .
\end{aligned}
$$

From this we see that $1.7 \%$ of the flux reflected by the sample undergoes interreflection with the shield. To find out what fraction of the flux contained in the sphere is received by the shield after the flux has been uniformly distributed over the entire sphere surface, we divide the sphere into hemispheres by a plane containing the shield. Let $A_{h 1}$ be the area of the hemisphere facing the front side of the shield and containing the sample port and $A_{h 2}$ the area of the hemisphere facing the back side of the shield. All the flux leaving $A_{\text {shl }}$, the front side of the shield, has to strike A or $\mathrm{F}_{\operatorname{sh} 1, \mathrm{~h} 1}=1$.

From the law of reciprocity, we know that

$$
\mathrm{A}_{\operatorname{sh} 1} \mathrm{~F}_{\operatorname{sh} 1, \mathrm{~h} 1}=\mathrm{A}_{\mathrm{h} 1} \mathrm{~F}_{\mathrm{h} 1, \operatorname{sh} 1}
$$

or

$$
\mathrm{F}_{\mathrm{h} 1, \mathrm{sh} 1}=\frac{\mathrm{A}_{\text {sh } 1}}{\mathrm{~A}_{\mathrm{h} 1}} \mathrm{~F}_{\text {sh } 1, \mathrm{~h} 1}=\frac{\mathrm{A}_{\text {sh } 1}}{\mathrm{~A}_{\mathrm{h} 1}} \text {. }
$$

For the same reason

$$
\mathrm{F}_{\mathrm{h} 2, \mathrm{sh} 2}=\frac{\mathrm{A}_{\operatorname{sh} 2}}{\mathrm{~A}_{\mathrm{h} 2}}
$$

Now if $\Phi$ is the total $f 1$ ux leaving the sphere wal1, then approximately $\Phi / 2$ is contained in each hemisphere. The $f 1$ ux received by $A_{\operatorname{sh} 1}$ is

$$
\frac{\Phi}{2} \frac{A_{\text {sh } 1}}{A_{h 1}}=\frac{\Phi}{2} \frac{A_{s h}}{A / 2}=\Phi \frac{A_{s h}}{A},
$$

where $A_{s h} / A=0.00031$. This means that only $0.03 \%$ of the flux contained in the sphere, after the flux has been uniformly distributed, is incident on the front side of the shield. Almost al1 shield losses occur on this side of the shield since $A_{\text {sh } 1}$ views all openings except a part of $A_{4}$ (see figures 4 and 5). Therefore, the exact losses due to the shield are taken into account only before the flux has been diffused by the sphere wall. After the flux has been distributed over the entire sphere surface, we treat the shield as an increase in surface area of the sphere of $2 A_{\text {sh }}$ with a reflectance of $\rho_{\text {sh }}$.

The flux contained in the sphere after one wall reflection was

$$
\begin{aligned}
\Phi_{1} & =\rho_{\mathrm{w}} \rho_{s} \Phi_{0}\left\{1-\mathrm{F}_{i, 126}-\mathrm{F}_{i, s h}\left[1-\rho_{s h}\left(1-\mathrm{F}_{s h, 1236 s}\right)\right]\right\}+\rho_{s}^{2} \Phi_{o} F_{i, s h} \rho_{s h} F_{s h, s} \\
& =\rho_{w} \rho_{s} \Phi_{0}\left\{1-F_{i, 126}-F_{i, s h}\left[1-\rho_{s h}\left(1-F_{s h, 1236 s}\right)\right]+\rho_{s} \frac{\rho_{s h}}{\rho_{w}} F_{i, s h} F_{s h, s}\right\} .
\end{aligned}
$$

Now we define an average sphere reflectance $\bar{p}_{\mathrm{w}}$ such that

$$
\bar{\rho}_{W}\left(A+2 A_{s h}\right)=\rho_{s h} 2 A_{s h}+\rho_{w}\left[A-\left(A_{1}+A_{2}+A_{3}+A_{4}+A_{s}\right)\right]+\rho_{s} A_{s}
$$

or

$$
\vec{p}_{w}=\frac{1}{A+2 A_{s h}}\left\{2 A_{s h} \rho_{s h}+p_{w}\left[A-\left(A_{1}+A_{2}+A_{3}+A_{4}+A_{s}\right)\right]+p_{s} A_{s}\right\} \text {. }
$$

Then, if $\Phi_{n}$ is the flux contained in the sphere after $n$ reflections, the flux after $n+1$ reflections is

$$
\Phi_{\mathrm{n}}+1=\bar{\rho}_{\mathrm{w}} \Phi_{\mathrm{n}} \text {. }
$$

After any reflection $n$ the fraction $A_{v} / A$ of the flux $\Phi_{n}$ is incident on $A_{v}$. The refore, the flux received by $\mathrm{A}_{\mathrm{v}}$ is

$$
\begin{aligned}
\Phi_{\mathrm{v}} & =\sum_{\mathrm{n}=1}^{\infty} \Phi_{\mathrm{v}, \mathrm{n}} \\
& =\Phi_{\mathrm{v}, 1}+\Phi_{\mathrm{v}, 2}+\ldots .
\end{aligned}
$$




$$
\begin{aligned}
& =\Phi_{\mathrm{v}, 1}+\frac{\mathrm{A}}{\mathrm{A}} \sum_{\mathrm{n}=2}^{\infty} \Phi_{\mathrm{n}} \\
& =\Phi_{\mathrm{v}, 1}+\frac{\mathrm{A}_{\mathrm{v}}}{\mathrm{A}}\left[\Phi_{1} \bar{\rho}_{\mathrm{w}}+\Phi_{1} \overline{\rho_{\mathrm{w}}^{2}}+\Phi_{1} \bar{\rho}_{\mathrm{w}}^{3}+\ldots .\right] \\
& =\Phi_{\mathrm{v}, 1}+\frac{\mathrm{A}_{\mathrm{v}}}{\mathrm{A}} \bar{\Phi}_{1} \bar{\rho}_{\mathrm{w}} \frac{1}{1-\rho_{\mathrm{w}}} .
\end{aligned}
$$

There fore

$$
\begin{aligned}
& \underline{\delta}_{v}=\frac{A}{A} \rho_{w} \rho_{s} \tilde{z}_{0}\left\{1-F_{i, 126}-F_{i, s h}\left[1-\rho_{s h}\left(1-F_{s h, 1236 s}\right)\right]\right\}
\end{aligned}
$$

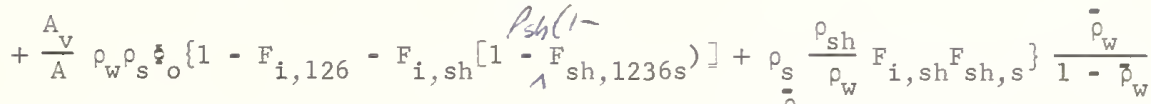

$$
\begin{aligned}
& =\frac{A_{\mathrm{V}}}{\mathrm{A}} \rho_{\mathrm{W}} \rho_{\mathrm{S}}{ }_{0}\left\{1-\mathrm{F}_{i, 126}-F_{i, s h}\left[1-\rho_{s h}\left(1-\mathrm{F}_{s h, 1236 \mathrm{~s}}\right)\right]\right\} \times\left\{1+\frac{\bar{\rho}_{\mathrm{W}}}{1-\bar{\rho}_{\mathrm{W}}}(1\right. \\
& \left.\left.+\frac{P_{S} \rho_{s h} F_{i, s h} F_{s h, s}}{P_{w}\left\{1-F_{i, 126}-F_{i, s h}\left[1-P_{s h}\left(1-F_{s h, 1236 s}\right)\right]\right\}}\right)\right\} .
\end{aligned}
$$

When we check the order of magnitude of the term

$$
\frac{\rho_{s} \rho_{s h} F_{i, s h}{ }^{F}{ }_{S h, s}}{\left.\rho_{W}\left\{1-F_{i, 126}-F_{i, s h}{ }^{L 1}-\rho_{s h}\left(1-F_{s h, 1236 s}\right)\right]\right\}}
$$

we see that it is much smaller than 1 and therefore can be neglected. (The maximum value of this term is about 0.0004 ). Neglecting this term we can further simplify the expression to

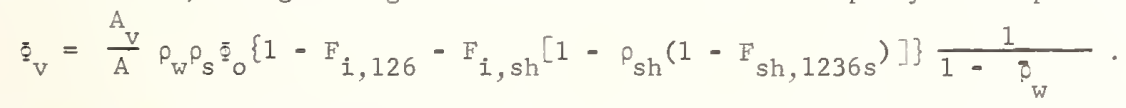

Since we assume that the signal from the detector-amplifier-recorder system is proportional to the radiance of $\mathrm{A}_{\mathrm{v}}$, $\mathrm{S}_{1}$, the signal when the beam is first incident on the sample, becomes

$$
S_{1}=K \frac{1}{\pi A} \rho_{W}^{2} \rho_{S} \Phi_{0}\left\{1-F_{i, 126}-F_{i, s h}\left[1-p_{s h}\left(1-F_{s h, 1236 s}\right)\right]\right\} \frac{1}{1-\bar{p}_{W}} .
$$

where $\mathrm{K}$ is a proportionality constant.

To measure $\bar{g}_{0}$, the beam is directed onto an area $A_{W}$ of the sphere surface with an unobstructed view of $A_{v}$. Reflected off the sphere wall is $p_{W} \bar{g}$ of which the fraction $A_{v} / A_{\text {is }}$ received by $A_{V}$. Thus

$$
\bar{\varrho}_{\mathrm{V}, 1}=\frac{\mathrm{A}}{\mathrm{A}} \rho_{\mathrm{W}} \bar{\sigma}_{0} \text {. }
$$

Of the flux $\rho_{w}{ }^{\circ}$, the fraction $F_{w, 124}$ is lost through the openings $A_{1}, A_{2}$, and $A_{4}$. (The opening $A_{3}$ and the sample area are screened off by the shield.) Another fraction $F_{w, s h}$ is incident on the backside of the shield A $A_{\text {sh2 }}$ of which $\rho_{s h} F_{w, s h}$ is reflected. The flux reflected off the shield is therefore

$$
\rho_{\text {Sh }} \rho_{W} F_{W}, \operatorname{sh} 2{ }^{\Phi}{ }_{0}
$$

A portion of this flux is in turn lost through that part of $\mathrm{A}_{4}$ which is viewed by $\mathrm{A}_{\text {sh2 }}$. This flux is therefore

$$
P_{\text {sh }} \rho_{w} \varrho_{0} F_{w}, \operatorname{sh} 2{ }^{F} \operatorname{sh} 2,4^{\circ}
$$

The flux incident on the sphere wall directly is

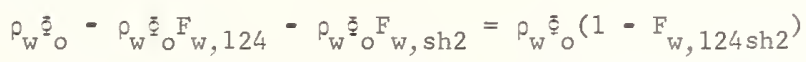

of which the fraction $\mathrm{A}_{\mathrm{V}} / \mathrm{A}$ is again incident on $\mathrm{A}_{\mathrm{V}}$. In addition to this, there is a small amount of flux

$$
\rho_{\mathrm{sh}} \rho_{\mathrm{w}}{ }_{\mathrm{O}} \mathrm{F}_{\mathrm{w}}, \operatorname{sh} 2^{\mathrm{F}} \operatorname{sh} 2, \mathrm{v}
$$

${ }^{1} A_{W}$ is located at the position of the comparison standard (see Figure 4 ). 
which is reflected off the shield and then received by $A_{V}$. Thus

$$
\begin{aligned}
& \Phi_{v, 2}=\frac{A_{v}}{A} \rho_{W}^{2} \Phi_{0}\left(1-F_{w}, 124 \operatorname{sh} 2\right)+\rho_{w} \rho_{s h} \Phi_{0} F_{w}, \operatorname{sh} 2{ }^{F} \operatorname{sh} 2, v \\
& =\frac{A_{V}}{A} \rho_{W}^{2} \Phi_{0}\left(1-F_{w, 124 s h}+\frac{A}{A} \frac{\rho_{s h}}{\rho_{w}} F_{w, ~ s h 2}{ }^{F} \operatorname{sh} 2, v\right) \text {. }
\end{aligned}
$$

The flux remaining after two wall reflections is

$$
\begin{aligned}
\Phi_{2} & =\rho_{\mathrm{w}}^{2} \Phi_{0}\left(1-F_{\mathrm{w}, 124 \mathrm{sh} 2}\right)+\rho_{\mathrm{w}} \rho_{\mathrm{sh}} \Phi_{\mathrm{O}} \mathrm{F}_{\mathrm{w}, \mathrm{sh} 2}\left(1-\mathrm{F}_{\mathrm{sh} 2,4}\right) \\
& =\rho_{\mathrm{w}}^{2} \Phi_{\mathrm{O}}\left[1-\mathrm{F}_{\mathrm{w}, 124 \mathrm{sh} 2}+\frac{\rho_{\mathrm{sh}}}{\rho_{\mathrm{w}}} \mathrm{F}_{\mathrm{w}, \mathrm{sh} 2}\left(1-\mathrm{F}_{\operatorname{sh} 2,4}\right)\right] .
\end{aligned}
$$

From this point on we assume that the flux is uniformly distributed over the whole surface of the sphere and treat the following reflections in the same manner as before. Therefore the sum of all the flux incident on $A_{v}$ after the second wall reflection is

$$
\sum_{n=3}^{\infty} \Phi_{v, n}=\frac{A_{v}}{A} \Phi_{2} \frac{\bar{p}_{w}}{1-\bar{p}_{w}}
$$

and therefore $\Phi_{\mathrm{v}}$ becomes

$$
\begin{aligned}
& \Phi_{V}=\frac{A}{A} \rho_{w} \Phi_{0}+\frac{A}{A} \rho_{w}^{2} \Phi_{0}\left(1-F_{w, 124 s h}+\frac{A}{A} \frac{\rho_{s h}}{\rho_{w}} F_{w, s h} F_{\operatorname{sh} 2, v}\right) \\
& +\frac{A_{V}}{A} \rho_{w}^{2} \Phi_{0}\left[1-F_{w, 124 s h 2}+\frac{\rho_{s h}}{\rho_{w}} F_{w, s h}\left(1-F_{s h 2,4}\right)\right] \frac{\bar{\rho}_{w}}{1-\bar{\rho}_{w}} \\
& =\frac{A_{V}}{A} \rho_{w} \Phi_{0}\left\{1+\rho_{w}\left[\left(1-F_{w, 124 s h 2}\right) \frac{1}{1-\bar{\rho}_{W}}+\frac{\rho_{s h}}{\rho_{w}} F_{w, s h}<\frac{A}{A_{v}} F_{s h 2, v}\right.\right. \\
& \left.\left.\left.+\frac{\bar{\rho}_{w}}{1-\bar{\rho}_{w}}\left(1-F_{\operatorname{sh} 2,4}\right)\right\rangle\right]\right\} \text {. }
\end{aligned}
$$

Hence $\mathrm{S}_{2}$, the signal when the beam is first incident on the sphere wall, becomes

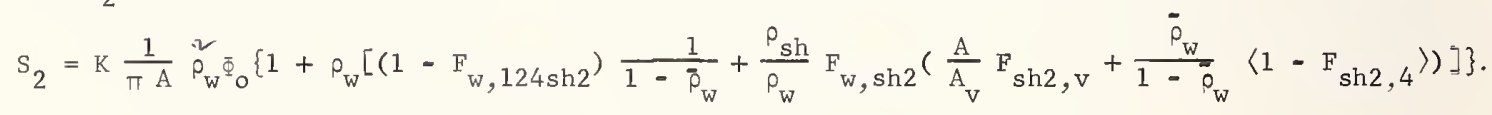

The ratio of these two signals $S_{1} / S_{2}$ is therefore

$$
\frac{S_{1}}{S_{2}}=\rho_{s} \frac{1-F_{i, 126}-F_{i, s h}\left[1-\rho_{s h}\left(1-F_{s h, 1236 s}\right)\right]}{\left(1-\tilde{\rho}_{w}\right)} x
$$

$$
\left\{1+\rho_{w}\left[\left(1-F_{w, 124 s h 2}\right) \frac{1}{1-\bar{\rho}_{W}}+\frac{\rho_{s h}}{\rho_{W}} F_{w, s h 2}\left\langle\frac{A}{A_{V}} F_{s h 2, v}+\frac{\bar{\rho}_{W}}{1-\bar{\rho}_{W}}\left(1-F_{\operatorname{sh} 2,4}\right)\right\rangle\right]\right\}
$$

or

$$
\rho_{s}=\frac{s_{1}}{s_{2}} c
$$

where $\mathrm{C}$ is a correction factor.

The magnitude of the error, in percent of the correct value, is given by

$$
\frac{\rho_{S, \text { correct }}-\rho_{S, \text { measured }}}{\rho_{\mathrm{S}, \text { correct }}} \quad 100=\frac{\frac{S_{1}}{S_{2}} \mathrm{C}-\frac{S_{1}}{S_{2}}}{\frac{S_{1}}{S_{2}} C} 100=\frac{C-1}{C} 100 .
$$


For a particular geometry $\mathrm{C}$ is a function of $\rho_{\mathrm{w}}, \rho_{\mathrm{S}}$, and $\rho_{\mathrm{sh}}$.

In this case the shield is coated with the same material as the surface of the sphere and therefore

$$
\rho_{\text {sh }}=\rho_{\text {w }} \text {. }
$$

A11 the configuration factors were calculated.

$$
\begin{array}{ll}
F_{i, 126} & =0.0051 \\
F_{i, s h} & =0.017 \\
F_{\text {sh,1236s }} & =0.00575 \\
F_{w, 124 s h 2} & =0.0072 \\
F_{W, s h 2} & =0.0017 \\
F_{\text {sh2,v }} & =0.00672 \\
F_{\text {sh2 } 2,4} & =0.0079
\end{array}
$$

In addition we have

$$
\bar{p}_{w}=0.9939 p_{w}+0.00033 \rho_{s}
$$

and

$$
\frac{\mathrm{A}_{\mathrm{V}}}{\mathrm{A}_{\mathrm{V}}}=155.35
$$

Using these values, we obtain

$$
C=\frac{1.00075-\left(0.00033 \rho_{\mathrm{S}}+0.00017 \rho_{\mathrm{w}}\right)}{0.9779+0.0169 \rho_{\mathrm{w}}} .
$$

Table I shows the calculated correction factor $\mathrm{C}$ and the error in percentage of the correct value as a function of wall reflectance for a sample reflectance of 0.1 . Table II gives the same values for a sample reflectance of 0.95 .

Table I

Correction Factor $\mathrm{C}$ and the Error in Percent of the Correct

Value for $\rho_{S}=0.1$ for the Case of a Perfectly Diffuse Sample

\begin{tabular}{lccccc}
\hline Sample Reflectance $\rho_{\mathrm{S}}$ & \multicolumn{5}{c}{0.1} \\
\hline Wall Reflectance $\rho_{\mathrm{W}}$ & 0.4 & 0.6 & 0.8 & 0.9 & 1.0 \\
Correction Factor C & 1.016 & 1.013 & 1.009 & 1.008 & 1.006 \\
$\frac{\mathrm{C}-1}{\mathrm{C}} 100 \mathrm{~L} \%$ & 1.59 & 1.25 & 0.92 & 0.75 & 0.57 \\
\hline
\end{tabular}

Table II

Correction Factor $\mathrm{C}$ and the Error in Percent of the Correct Value for $\rho_{S}=0.95$ for the Case of a Perfectly Diffuse Sample

\begin{tabular}{llllll}
\hline Sample Reflectance $P_{\mathrm{S}}$ & \multicolumn{5}{c}{0.95} \\
\hline Wall Reflectance $P_{\mathrm{W}}$ & 0.4 & 0.6 & 0.8 & 0.9 & 1.0 \\
Correction Factor C & 1.016 & 1.012 & 1.009 & 1.007 & 1.0055 \\
$\frac{\mathrm{C}-1}{\mathrm{C}} 100[\%]$ & 1.57 & 1.19 & 0.89 & 0.71 & 0.55 \\
\hline
\end{tabular}




\subsubsection{Perfectly Specular Sample}

Again let $\Phi_{0}$ be the flux which is initially incident on the sample. Then $\rho_{s} \Phi_{0}$ is the flux which is reflected by the sample of reflectance $\rho_{s}$ in a specular manner and strikes the sphere wall. (From Figure 4 it can be seen that no flux will be lost through any of the openings and none will be received by the shie1d.) The flux that leaves the sphere wall then is

$$
\rho_{W} \rho_{S} \Phi_{0}
$$

of which the fraction $A_{v} / A$ is incident on the detector's field of view. Therefore,

$$
\Phi_{v, 1}=\frac{{ }_{v}}{A} \rho_{w} \rho_{s} \Phi_{0} .
$$

A fraction of the flux that leaves the sphere wall is incident on the sample and is specularly reflected towards the entrance port. If $A_{i l}$ is the area where the specularly reflected beam from the sample strikes the sphere wall initially, then

$$
\rho_{w} \rho_{s} \Phi_{0} F i 1, s
$$

is the flux which is in turn specularly reflected towards $A_{1}$. This flux appears to originate at the image of $A_{i 1}$ which we call $A_{i 1}$ as seen in the mirrorlike sample. The fraction of the flux that leaves $A_{i 1}$ and is lost through $A_{1}$ due to specular reflection by the sample is therefore determined by the configuration factor from the image of $A_{i 1}$ to $A_{1}$ or $F \overline{i 1}, 1$ (See Figure 6.)

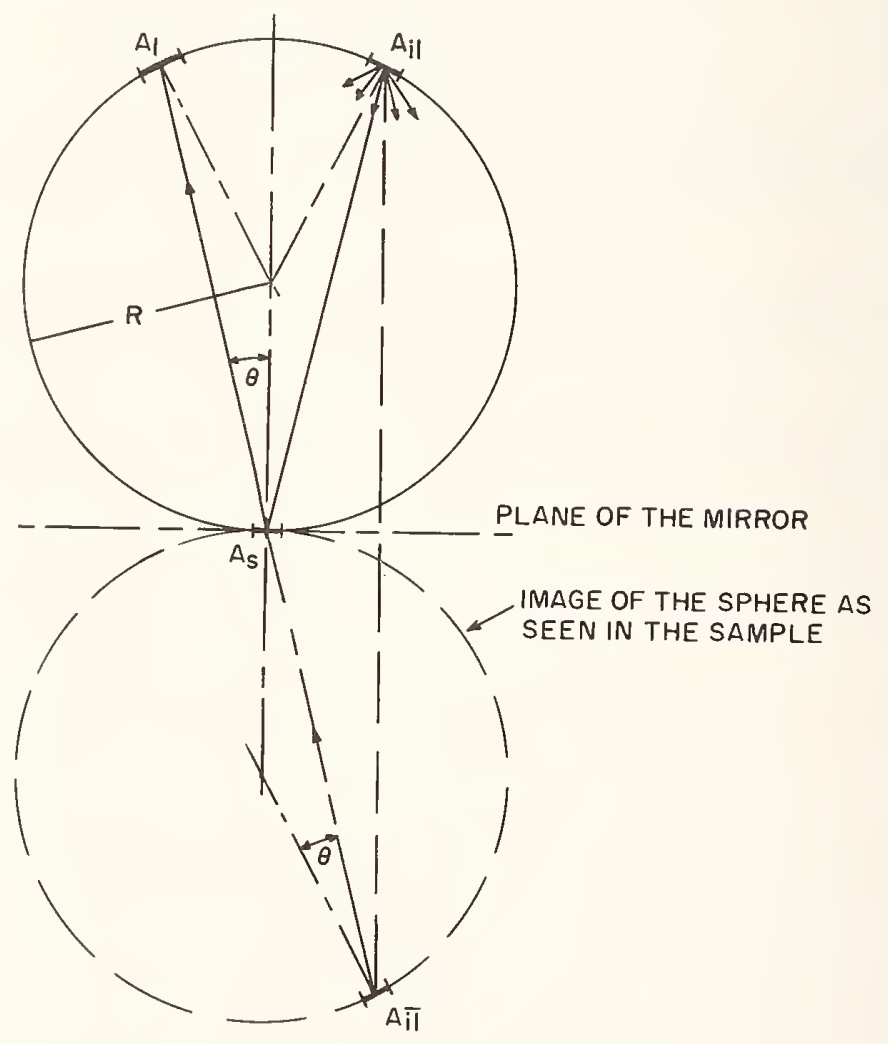

Figure 6. Image of the Integrating Sphere As Seen in a Perfect 1y Specular Sample 
Since the areas involved are very small compared with the distance, it can be assumed that the configuration factor is essentially the same for any point of $A_{i 1}$ and $A_{1}$ and therefore

$$
F \overline{i 1,1}=\frac{\cos \theta_{i 1} \cos \theta_{1}}{\pi^{2}}
$$

where $\theta_{i 1}$ is the angle between the normal to $A_{i 1}$ and the line of sight, $\theta_{1}$ the analogous angle for $A_{1}$ and $B$ the distance separating $A_{1}$ and $A_{i 1}$.

From Figure 6 it can be seen that

$$
\cos \theta_{i 1}=\cos \theta_{1}=\cos \theta
$$

and

$$
B=2(2 R \cos \theta) \text {. }
$$

With this

$$
\mathrm{F}_{i 1,1}=\frac{\cos ^{2} \theta}{\pi 16 \mathrm{R}^{2} \cos \theta^{2}} \mathrm{~A}_{1}=\frac{\mathrm{A}_{1}}{4\left(4 \pi \mathrm{R}^{2}\right)}=\frac{\mathrm{A}_{1}}{4 \mathrm{~A}}
$$

or the fraction of flux which is leaving $A_{i l}$ and is specularly reflected out the entrance port is equal to the area of the entrance port divided by four times the total sphere area. However, one restriction has to be observed. If; as in Figure 7, the entire entrance hole can be seen from A through the mirror then

$$
\mathrm{F}_{\mathrm{i} 1,1}=\frac{\mathrm{A}_{1}}{4 \mathrm{~A}} \text { as indicated. }
$$

If on the other hand the sample is so small that it restricts the sight from $A_{i 1}$ to $A_{1}$, as shown in Figure 8, then the configuration factor to use is not $A_{1} / 4 A_{\text {but }} F_{i 1}$, which is equal to $F_{i 1}, s=A_{s} / A_{\text {. }}$ The condition for $F=A_{1} / 4 A$, where $F$ is now used for the fraction specularly reflected out $A_{1}$, is

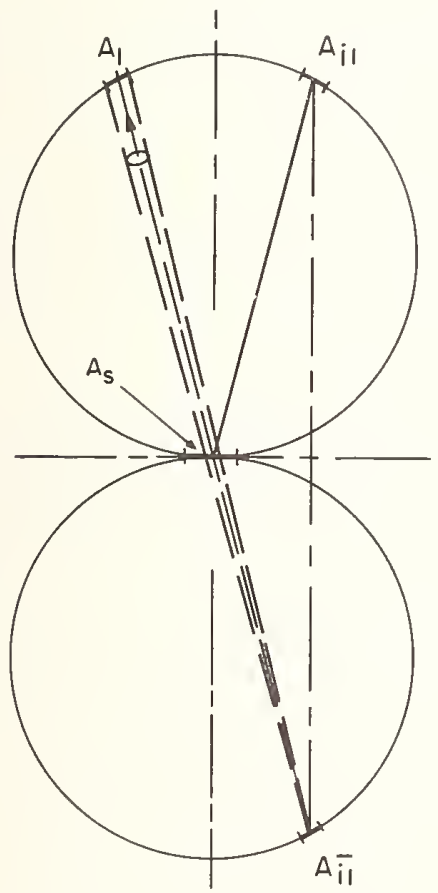

Figure 7. Sphere Geometry for $A_{1}<4 A_{s}$

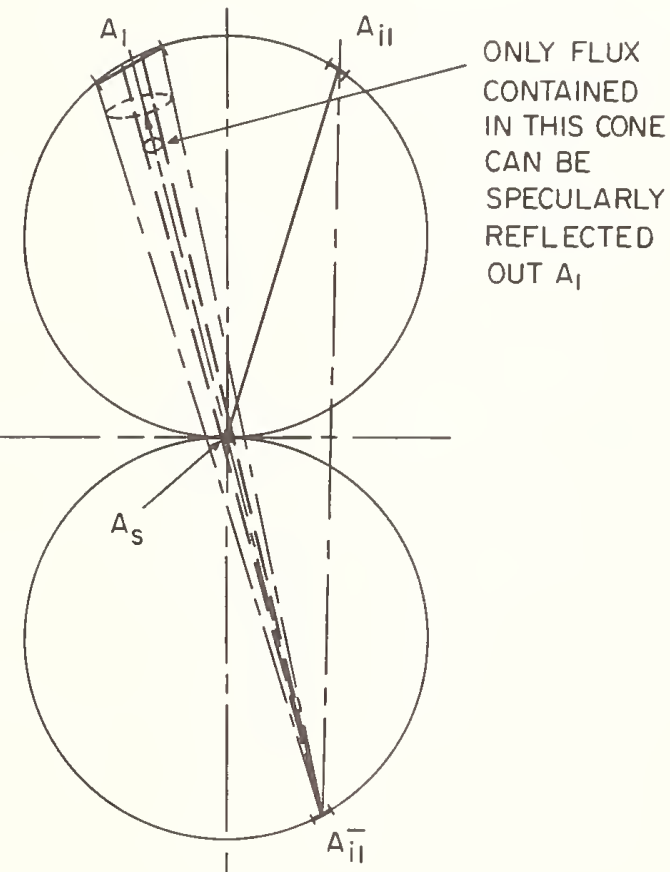

Figure 8. Sphere Geometry For $\mathrm{A}_{1}>4 \mathrm{~A}_{\mathrm{s}}$ 


$$
\frac{A_{1}}{4 A}<\frac{A_{S}}{A} \text { or } A_{1}<4 A_{S}
$$

and

$$
F=\frac{A_{S}}{A} \text { if } A_{1} \geq 4 A_{S} \text {. }
$$

If $A_{1}<4 A_{s}$ then $\rho_{w} \rho_{s} \Phi_{0} A_{1} / 4 \mathrm{~A}$ is lost through $A_{1}$. Since

$$
\rho_{W} \rho_{s}^{2} \Phi_{0} F_{i 1, s}=\rho_{W} \rho_{s}^{2} \Phi_{0} \frac{A_{s}}{A}
$$

was reflected specularly towards the entrance port, therefore

$$
\rho_{\mathrm{w}} \rho_{s}^{2} \Phi_{0}\left[\frac{\mathrm{A}_{S}}{\mathrm{~A}}-\frac{\mathrm{A}_{1}}{4 \mathrm{~A}}\right]=\rho_{\mathrm{w}} \rho_{\mathrm{S}}^{2} \Phi_{0} \frac{1}{\mathrm{~A}}\left(\mathrm{~A}_{\mathrm{S}}-\frac{1}{4} \mathrm{~A}_{1}\right)
$$

is incident on the sphere wall around the entrance port.

If $A_{1} \leq 4 A_{s}$ then all of the specularly reflected flux is lost through $A_{1}$. In this case the flux lost is

$$
\rho_{S} \rho_{W} \Phi_{0} \frac{A_{S}}{A} \text {. }
$$

(This includes the flux absorbed at the sample.) For our sphere $A_{1}=6.413 \mathrm{~cm}^{2}\left(0.994\right.$ in $\left.^{2}\right)$ and $A_{s}=$ $1.264 \mathrm{~cm}^{2}\left(0.196 \mathrm{in}^{2}\right)$; therefore, $\mathrm{A}_{1}>4 \mathrm{~A}_{\mathrm{s}}$ and the flux lost is $p_{\mathrm{w}} p_{\mathrm{s}} \Phi_{\mathrm{o}} \mathrm{A}_{\mathrm{s}} / \mathrm{A}$.

Once the flux is uniformly distributed over the entire surface area of the sphere, we need additional information to calculate the hole loss caused by specular reflection at the sample. Now it is necessary to know the area from which diffusely reflected flux is able to reach $A_{1}$ via the specular sample. Let this area be $A_{i 2}$. Now, if $\Phi$ is the flux uniformly distributed over the sphere wall then $\left(A_{i 2} / A\right) \Phi$ is the flux leaving the area $A_{i 2}$. Of this flux the fraction $A_{s} / A$ is received by the specular sample and reflected towards the entrance hole. This reflected flux strikes the sphere wall within an area centered around the entrance port. If $\mathrm{A}_{13}$ is this irradiated area, then the fraction lost out the entrance port is $A_{1} / A_{i 3}$. Hence the additional loss through the entrance port caused by the specular sample becomes

$$
\frac{A_{1}}{A_{i 3}} \frac{A_{i 2} A_{S}}{A^{2}} \Phi .
$$

In the same way, there exists another portion of flux which is specularly reflected into the detector port and absorbed by the light trap without reaching the detector. This flux can be expressed as

$$
\frac{A_{2}}{A_{i 5}} \frac{A_{i 4} A_{S}}{A^{2}} \Phi,
$$

where $A_{i 5}$ and $A_{i 4}$ are the corresponding areas to $A_{i 3}$ and $A_{i 2}$. The areas $A_{i 2}$ and $A_{i 3}$ (or $A_{i 5}$ and $A_{i 4}$ ) can be found graphically as shown in Figure 9. The method corresponds to finding the area on the sphere which is irradiated by the mirror $A_{s}$ if the entrance port is replaced by a diffusely emitting source of equal area and then in turn finding the irradiated area around the entrance port due to the diffusely reflecting area $\mathrm{A}_{12}$ or $\mathrm{A}_{14}$ respectively.

Now assume that after $n$ reflections the flux is uniformly distributed over the sphere wall. Let this flux be $\Phi_{n}$. Then the problem is to calculate $\Phi_{n}+1$.

of the flux $\Phi_{n}$ the fraction (1/A) $\left(A_{1}+A_{2}+A_{3}+A_{4}\right)$ is lost through the openings direct $1 y$. An additional fraction,

$$
\frac{A_{S}}{A^{2}}\left[A_{1} \frac{A_{12}}{A_{i 3}}+A_{2} \frac{A_{14}}{A_{i 5}}\right],
$$

is lost due to specular reflections of the sample. (No flux can be specularly reflected out $A_{3}$ since $A_{3}$ is in the same plane as $A_{5}$ and no flux can be specularly reflected out $A_{4}$ since $A_{4}$ is arranged symmetrically around the normal to $\mathrm{A}_{\mathrm{S}}$. Therefore for any part of $\mathrm{A}_{4}$ the corresponding area from which the flux would have to originate is again a part of the opening $A_{4}$ with an effective $p=0$.) (See 
Figure 5.) With this the total hole loss becomes

$$
\frac{1}{A}\left[A_{1}+A_{2}+A_{3}+A_{4}+\frac{A_{S}}{A}\left(A_{1} \frac{A_{i 2}}{A_{i 3}}+A_{2} \frac{A_{i 4}}{A_{i 5}}\right)\right] \Phi_{n} .
$$

The portion $\left(A_{S} / A\right)_{n}$ is incident on the sample which absorbs

$$
\frac{A_{S}}{A}\left(1-\rho_{S}\right) \Phi_{n} \text {. }
$$

Another part, $2 \mathrm{~A}_{\mathrm{sh}} / \mathrm{A}$, is received by the shield which absorbs the flux

$$
\frac{2 A_{s h}}{A}\left(1-P_{s h}\right) \Phi_{n} \text {. }
$$

The remaining flux,

$$
\Phi_{n}-\left[\frac{1}{A}\left(A_{1}+A_{2}+A_{3}+A_{4}\right)+\frac{A s}{A}+\frac{2 A_{s h}}{A}\right] \Phi_{n},
$$

is incident on the sphere wall which absorbs

$$
\Phi_{n}\left\{1-\frac{1}{A}\left[A_{1}+A_{2}+A_{3}+A_{4}+A_{s}+2 A_{s h}\right]\right\}\left(1-\rho_{W}\right) .
$$

Therefore the flux contained in the sphere after $n+1$ wall reflections becomes

$$
\begin{aligned}
& \Phi_{n+1}=\Phi_{n}-\frac{1}{A}\left[A_{1}+A_{2}+A_{3}+A_{4}+\frac{A_{S}}{A}\left(A_{1} \frac{A_{i 2}}{A_{i 3}}+A_{2} \frac{A_{i 4}}{A_{i 5}}\right)\right]_{n} \\
& -\frac{A_{S}}{A}\left(1-\rho_{S}\right) \Phi_{n}-\frac{2 A}{A}\left(1-\rho_{s h}\right) \Phi_{n}-\frac{1}{A}\left\{A-\left[A_{1}+A_{2}\right.\right. \\
& \left.\left.+\mathrm{A}_{3}+\mathrm{A}_{4}+\mathrm{A}_{\mathrm{s}}+2 \mathrm{~A}_{\mathrm{sh}}\right]\right\}\left(1-\rho_{\mathrm{w}}\right) \Phi_{\mathrm{n}} \\
& \Phi_{n+1}=\Phi_{n}\left\{1-\frac{1}{A}\left[A_{1}+A_{2}+A_{3}+A_{4}+\frac{A_{S}}{A}\left(A_{1} \frac{A_{i 2}}{A_{i 3}}+A_{2} \frac{A_{i 4}}{A_{i 5}}\right)\right]-\frac{A_{S}}{A}\left(1-\rho_{s}\right)\right. \\
& -\frac{2 A_{s h}}{A}\left(1-\rho_{s h}\right)-\frac{1}{A}\left\{A-\left[A_{1}+A_{2}+A_{3}+A_{4}+A_{s}+2 A_{s h}\right]\right\}\left(1-\rho_{w}\right)
\end{aligned}
$$

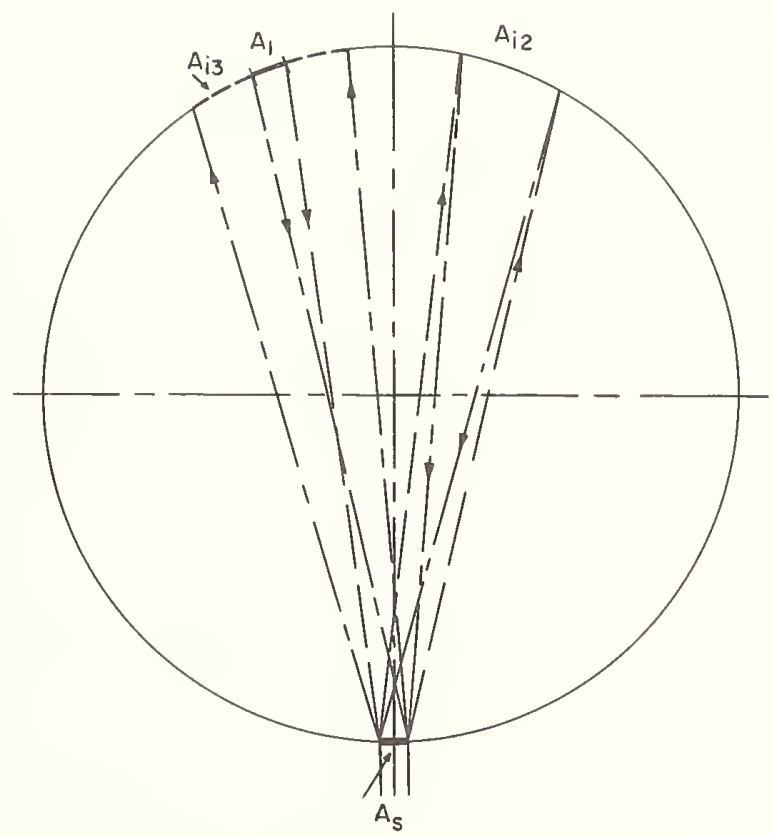

Figure 9. Graphical Method of Finding the Areas Necessary to Compute the Additional Hole Losses 


$$
\begin{aligned}
\Phi_{n+1}=\Phi_{n}\left\{\rho_{w}[1\right. & \left.-\frac{1}{A}\left(A_{1}+A_{2}+A_{3}+A_{4}+A_{s}+2 A_{s h}\right)\right]-\frac{A_{s}}{A^{2}}\left(A_{1} \frac{A_{12}}{A_{i 3}}+A_{2} \frac{A_{i 4}}{A_{i 5}}\right) \\
+ & \left.\frac{2 A_{s h}}{A} \rho_{s h}+\frac{A_{s}}{A} \rho_{s}\right\} .
\end{aligned}
$$

Now we define an effective sphere reflectance such that $p_{\text {eff }}=\Phi_{n}+1 / \Phi_{n}$ where $p_{\text {eff }}$ can be calculated from the above equation.

It was previously shown that ${ }_{\mathrm{v}, 1}$ is given by

$$
\Phi_{v, 1}=\frac{A}{A} P_{w} p_{s} \Phi_{0}
$$

The flux left after one wall reflection is $p_{w} p_{s} \Phi_{0}$. Of that, the fraction

$$
\frac{1}{A}\left(A_{1}+A_{2}+A_{3}+A_{4}\right)
$$

is lost out openings directly. As discussed earlier an additional hole loss occurs by specular reflection, and by absorption at the sample which is given by

$$
\rho_{w} \rho_{s} \Phi_{0} \frac{A_{s}}{A} \text {. }
$$

Another fraction, $F_{i 2, s h}$, is incident on the shield which absorbs $F_{i 2, s h}\left(1-p_{s h}\right)$ of it. The remaining flux is received by the sphere wall which absorbs the fraction $\left(1-p_{W}\right)$ of it. The fraction received by the wall is

$$
\begin{aligned}
\rho_{\mathrm{w}} \rho_{s} \Phi_{0} & -\frac{1}{A}\left(A_{1}+A_{2}+A_{3}+A_{4}\right) \rho_{w} \rho_{s} \Phi_{0}-\frac{A_{s}}{A} \rho_{w} \rho_{s} \Phi_{0}-F_{i 2, s h} \rho_{w} \rho_{s} \Phi_{0} \\
= & \rho_{W} \rho_{s} \Phi_{0}\left[1-\frac{1}{A}\left(A_{1}+A_{2}+A_{3}+A_{4}\right)-\frac{A_{s}}{A}-F_{i 2, s h}\right] .
\end{aligned}
$$

Therefore $\Phi_{\mathrm{v}, 2}$ is given by

$$
\Phi_{v, 2}=\frac{A_{v}}{A} \rho_{w_{s}}^{2} \rho_{s} \Phi_{0}\left[1-\frac{1}{A}\left(A_{1}+A_{2}+A_{3}+A_{4}+A_{s}\right)-F_{i 2, s h}\right] \text {. }
$$

The remaining flux after two reflections at the sphere wall is

$$
\begin{aligned}
& \Phi_{2}=\rho_{W} P_{s} \Phi_{0}-\frac{1}{A}\left(A_{1}+A_{2}+A_{3}+A_{4}+A_{s}\right) P_{W} P_{s} \Phi_{0}-F_{i 2, s h} P_{W} P_{s} \Phi_{0}\left(1-\rho_{s h}\right) \\
& -P_{\mathrm{w}} p_{\mathrm{s}} \Phi_{0}\left[1-\frac{1}{A}\left(A_{1}+A_{2}+A_{3}+A_{4}+A_{s}\right)-F_{i 2, s h}\right]\left(1-, P_{w}\right) \\
& \Phi_{2}=\rho_{\mathrm{w}}^{2} P_{\mathrm{s}} \Phi_{0}\left\{1-\frac{1}{A}\left(A_{1}+A_{2}+A_{3}+A_{4}+A_{s}\right)-F_{i 2, s h}\left(1-\frac{p_{s h}}{P_{w}}\right)\right\} \text {. }
\end{aligned}
$$

As in the previous cases, we assume from now on that the flux is evenly distributed over the surface of the sphere and that after any reflection $n$ the fraction $\left(A_{v} / A_{n} \Phi_{n}\right.$ is incident on $A_{v}$. Therefore

$$
\sum_{n=3}^{\infty} \Phi_{v, n}=\frac{A}{A}\left(\Phi_{2} p_{\text {eff }}+\Phi_{2} \rho_{\text {eff }}^{2}+\ldots\right)=\frac{A v}{A} \Phi_{2} \frac{\rho_{\text {eff }}}{1-\rho_{\text {eff }}}
$$

and $\sum_{\mathrm{n}=1}^{\infty} \Phi_{\mathrm{v}, \mathrm{n}}$ becomes

$$
\begin{aligned}
\frac{A_{v}}{A} \rho_{w} \rho_{s}^{\Phi} \Phi_{0} & +\frac{A}{A} \rho_{w}^{2} \rho_{s} \Phi_{0}\left[1-\frac{1}{A}\left(A_{1}+A_{2}+A_{3}+A_{4}+A_{s}\right)-F_{i 2, s h}\right]+\frac{A_{v}}{A} \rho_{w}^{2} \rho_{s} \Phi_{0}\{1 \\
& \left.-\frac{1}{A}\left(A_{1}+A_{2}+A_{3}+A_{4}+A_{s}\right)-F_{i 2, s h}\left(1-\frac{\rho_{s h}}{\rho_{w}}\right)\right\} \frac{\rho_{\text {eff }}}{1-\rho_{\text {eff }}} \\
=\frac{A}{A} \rho_{w} \rho_{s} \Phi_{0} & \left\{1+\rho_{w}\left[1-\frac{1}{A}\left(A_{1}+A_{2}+A_{3}+A_{4}+A_{s}\right)-F_{i 2, s h}\right]\right. \\
+ & \left.\rho_{w}\left[1-\frac{1}{A}\left(A_{1}+A_{2}+A_{3}+A_{4}+A_{s}\right)-F_{i 2, s h}\left(1-\frac{\rho_{s h}}{\rho_{w}}\right)\right] \frac{\rho_{\text {eff }}}{1-\rho_{\text {eff }}}\right\}
\end{aligned}
$$




$$
\begin{aligned}
& =\frac{A_{v}}{A} \rho_{w} \rho_{s} \Phi_{0}\left\{1+\rho_{w}\left[1-\frac{1}{A}\left(A_{1}+A_{2}+A_{3}+A_{4}+A_{s}\right)\right] \frac{1}{1-\rho_{\text {eff }}}\right. \\
& \left.-\rho_{\mathrm{w}} \mathrm{F}_{\mathrm{i} 2, \mathrm{sh}}\left[1+\left(1-\frac{\rho_{\mathrm{sh}}}{\rho_{\mathrm{w}}}\right) \frac{\rho_{\mathrm{eff}}}{1-\rho_{\text {eff }}}\right]\right\} \text {. }
\end{aligned}
$$

In our case

$$
\rho_{\text {sh }}=\rho_{w}
$$

and the signal from the detector becomes

$$
\mathrm{S}_{1}=\mathrm{K} \frac{1}{\pi \mathrm{A}} \rho_{\mathrm{w}}^{2} \rho_{\mathrm{s}} \Phi_{0}\left\{1-\rho_{\mathrm{w}}\left[1-\frac{1}{\mathrm{~A}}\left(\mathrm{~A}_{1}+\mathrm{A}_{2}+\mathrm{A}_{3}+\mathrm{A}_{4}+\mathrm{A}_{\mathrm{s}}\right)\right] \frac{1}{1-\rho_{\text {eff }}}=\rho_{\mathrm{w}} \mathrm{F}_{12, \mathrm{sh}}\right\} .
$$

When the beam is directed onto the sphere wall then $\Phi_{v, 1}$ and $\Phi_{v, 2}$ are exactly the same as in the diffuse case since the shield prevents the sample from viewing the irradiated area of the sphere wall, hence, no flux is incident on the sample before it has been reflected twice by the sphere wall. In fact, we can use the equation for $\Phi_{V}$ from page 14 if we replace the average wall reflectance $\bar{p}_{W}$ as used in the diffuse case by the effective sphere reflectance as defined in the specular case. Therefore $\mathrm{S}_{2}$, the signal when the beam is first incident on the sphere wall, is given by

$$
\begin{aligned}
\mathrm{S}_{2}=\mathrm{K} \frac{1}{\pi \mathrm{A}} \rho_{\mathrm{w}}^{2} \Phi_{0}\left\{1+\rho_{\mathrm{w}}[(1\right. & \left.-\mathrm{F}_{\mathrm{w}, 124 \mathrm{sh} 2}\right) \frac{1}{1-\rho_{\text {eff }}}+\mathrm{F}_{\mathrm{w}, \mathrm{sh} 2}\left(\frac{\mathrm{A}}{\mathrm{A}_{\mathrm{v}}} \mathrm{F}_{\mathrm{sh} 2, \mathrm{v}}\right. \\
& \left.\left.\left.+\frac{\rho_{\text {eff }}}{1-\rho_{\text {eff }}}\left\langle 1-\mathrm{F}_{\mathrm{sh} 2,4}\right\rangle\right)\right]\right\},
\end{aligned}
$$

and $\mathrm{S}_{1} / \mathrm{S}_{2}$, the ratio of the two signals, becomes

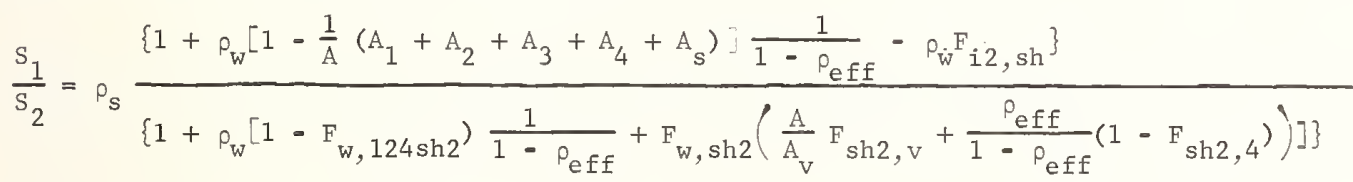

or

$$
\rho_{\mathrm{S}}=\frac{\mathrm{S}_{1}}{\mathrm{~S}_{2}} \mathrm{C}
$$

where the correction factor $C$ is defined by the equation (22).

In addition to the terms already used previously, we need $P_{\text {eff }}$ which was given by

$$
\begin{aligned}
\rho_{\text {eff }}=\rho_{\mathrm{w}}[1 & \left.-\frac{1}{A}\left(A_{1}+A_{2}+A_{3}+A_{4}+2 A_{s h}\right)\right]+\frac{2 A_{S h}}{A} \rho_{s h}+\frac{A_{S}}{A} \rho_{S} \\
& =\frac{A_{S}}{A^{2}}\left(A_{1} \frac{A_{i 2}}{A_{i 3}}+\frac{A_{i 4}}{A_{i 5}}\right) \\
= & 0.9939 \rho_{W}+0.00033 \rho_{S}-3.628 \times 10^{-7}
\end{aligned}
$$

when $\rho_{\text {sh }}$ was set equal to $\rho_{W}$.

From this we see that $\rho_{\text {eff }}$ cannot be distinguished from $\bar{\rho}_{W}$ and

$$
\rho_{\text {eff }}=\dot{\rho}_{w}=0.9939 \rho_{W}+0.00033 \rho_{s} \text {. }
$$

The other terms required are

$$
\frac{1}{A}\left(A_{1}+A_{2}+A_{3}+A_{4}+A_{S}\right)=0.0061
$$

and

$$
\mathrm{F}_{i 2, \mathrm{sh}}=5.62 \times 10^{-5} \text {. }
$$

With this the correction factor becomes 


$$
C=\frac{1-0.00033 \rho_{S}}{1+0.0166 \rho_{\mathrm{w}}+0.00033 \rho_{\mathrm{s}}-0.001592 \rho_{\mathrm{W}}^{2}}
$$

Table III gives the correction factor $\mathrm{C}$ and the correction in percent of the correct value for a perfectly specular sample as a function of wall reflectance for a sample reflectance of 0.1. Table IV shows the same values for a sample reflectance of 0.95 .

\section{Table IIT}

Correction Factor $\mathrm{C}$ and the Correction in Percent of the Correct

Value for a Sample Reflectance of 0.1 for the Case

of a Perfectly Specular Sample

\begin{tabular}{llllll}
\hline \multicolumn{5}{c}{$\rho_{\mathrm{S}}=0.1$} \\
\hline$\rho_{\mathrm{W}}$ & 0.4 & 0.6 & 0.8 & 0.9 & 1.0 \\
$\mathrm{C}$ & 0.9959 & 0.9957 & 0.9968 & 0.9979 & 0.9992 \\
$\frac{\mathrm{C}-1}{\mathrm{C}} 100[\%]$ & -0.41 & -0.43 & -0.32 & -0.21 & -0.08 \\
\hline
\end{tabular}

Table IV

Correction Factor $\mathrm{C}$ and the Correction in Percent of the Correct Value for a Sample Reflectance of 0.95 for the Case of a Perfectly Specular Sample

\begin{tabular}{llllll}
\hline \multicolumn{5}{c}{$\rho_{\mathrm{is}}=0.95$} \\
\hline$\rho_{\mathrm{W}}$ & 0.4 & 0.6 & 0.8 & 0.9 & 1.0 \\
$\mathrm{C}$ & 0.9955 & 0.9952 & 0.9965 & 0.9974 & 0.9987 \\
$\frac{\mathrm{C}-1}{\mathrm{C}} 100[\%]$ & -0.45 & -0.48 & -0.35 & -0.26 & -0.13 \\
\hline
\end{tabular}

Since it would be very difficult and tedious to calculate the correction factor for every real sample, the ratio $S_{1} / S_{2}$ was set equal to the directional hemispherical reflectance. In this case the correction factor for a perfect diffuser from Tables I and II or the curves of Figure 10 show the maximum error.

One additional comment about the relatively large errors for perfectly diffuse reflectors might be in order. These errors originate mainly from the fact that part of the flux reflected at polar angles around $75^{\circ}$ is intercepted by the shield and part of the flux reflected at polar angles around $85^{\circ}$ is trapped by the evacuation slots. Most real materials, even if they are considered to be good diffusers, reflect considerably less at these large polar angles than does a perfect diffuser. This means that for any real material the error is much smaller than that calculated on the basis of a perfect diffuser. Especially for metals which reflect predominantly in the specular direction even when roughened, the error will be closer to that for a perfect mirror than that for a perfect diffuser.

In any case, even if we take the error for a perfect diffuser as the upper limit and assuming a sphere wall reflectance of not less than 0.80 , we. see that the error will be less than $1 \%$ of the correct reflectance. Reflectance measurements to an accuracy of less than $1 \%$ are quite unusual even at room temperature. An accuracy of $1 \%$ or better had been obtained previously with an ellipsoidal mirror reflectometer [4], but this involved quite an elaborate correction procedure for several systematic 


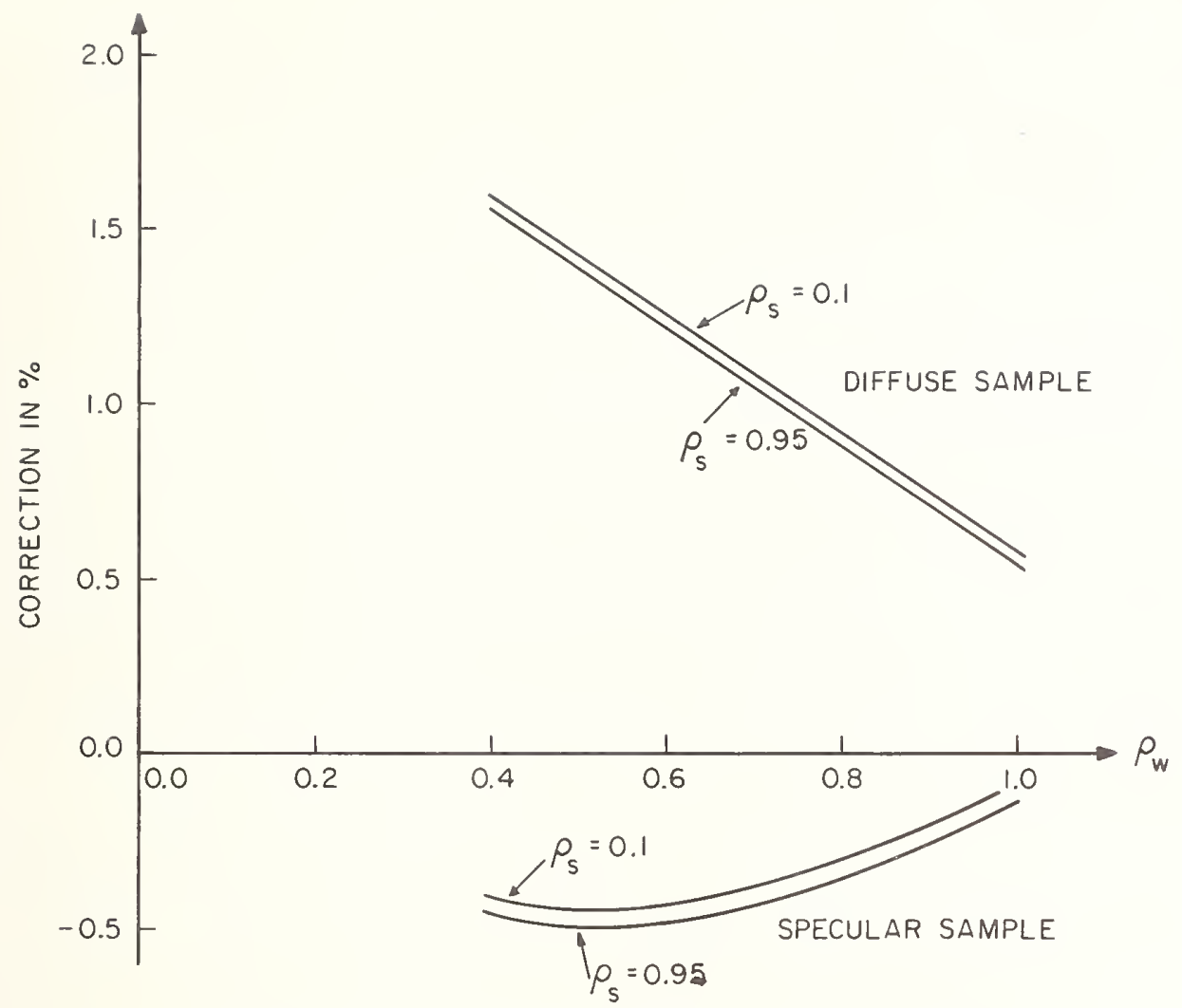

Figure 10. Correction in Percent of the Correct Value errors present which required additional measurements.

Since the accuracy of the integrating sphere reflectometer does not depend on the temperature of the sample, the conclusion is justified that, based on the theoretical analysis, this integrating sphere reflectometer is capable of measuring high temperature reflectance of any material to an accuracy of better than $1 \%$.

\section{List of Symbols}

Areas:
A
= Total surface area of the sphere $A=4 R^{2} \pi$
$\mathrm{A}_{1}$
= Area of the entrance port
$\mathrm{A}_{2}$
= Area of the detector port
$\mathrm{A}_{3}$
= Area of the ring space around the sample
$\mathrm{A}_{4}$
= Area of evacuation slots
$\mathrm{A}_{5}$
= Part of $\mathrm{A}_{4}$ screened off from the irradiated area of the sample
$\mathrm{A}_{6}$
$=A_{4}-A_{5}$
A $_{\text {sh }}$
= Area of one side of the shield
$\mathrm{A}_{\text {sh1 }}$
Area of the shield facing the sample
$\mathrm{A}_{\operatorname{sh} 2}$
= Area of the shield not facing the sample
A 1
= Area of the hemisphere facing $\mathrm{A}_{\text {sh1 }}$
An2
= Area of the hemisphere facing $\mathrm{A}_{\mathrm{sh} 2}$
= Area of the field of view of the detector
As
= Area of the sample 
$\mathrm{A}_{i} \quad=$ Part of $\mathrm{A}_{\mathrm{S}}$ initially irradiated by $\Phi_{0}$

$A_{i 1}=$ Area of the irradiated spot on the sphere due to the perfect specular reflection of $\Phi$ at the sample

$\mathrm{A}_{\mathrm{i} 1}=$ Image of $\mathrm{A}_{i 1}$ as seén in a perfectly specular sample

$A_{i 2}=$ Part of the sphere wall from which flux is able to reach $A_{1}$ due to perfect specular reflection at the sample

$A_{i 3}=$ Part of the sphere area centered around the entrance port, which is irradiated by flux leaving $A_{i 2}$ due to perfect specular reflection at the sample

$\mathrm{A}_{14}=$ Area analogous to $\mathrm{A}_{12}$ in connection with $\mathrm{A}_{2}$

$\mathrm{A}_{\text {i5 }}=$ Area analogous to $\mathrm{A}_{\text {i3 }}$ in connection with $\mathrm{A}_{2}$

Fluxes:

$\Phi=$ Flux [Watts]

$\Phi_{0}=$ Flux initially entering the sphere

$\Phi^{\prime} \quad=$ Flux reflected by the sample regardless of direction

$\Phi_{\mathrm{V}} \quad=$ Sum of $f 1 \mathrm{x}$ incident on the field of view of the detector after $\Phi_{0}$ has undergone an infinite number of reflections

$\Phi_{\mathrm{V}, \mathrm{n}}=$ Flux incident on $\mathrm{A}_{\mathrm{V}}$ after $\Phi_{\mathrm{O}}$ has undergone $\mathrm{n}$ reflections at the sphere wall

$\Phi_{\mathrm{n}}=$ Flux contained in the sphere after $\Phi_{0}$ has undergone $\mathrm{n}$ wall reflections Reflect ances:

$\rho_{\mathrm{S}} \quad=$ Directional - hemispherical reflectance of the sample correct $1 \mathrm{y}$ expressed as $\rho\left(12^{\circ} ; 2 \pi\right)$

$p_{\mathrm{w}} \quad=$ Reflectance of the sphere wall

$\rho_{\text {sh }}=$ Reflectance of the shield

$\bar{\rho}_{\mathrm{W}} \quad=$ Average sphere wall reflectance as defined on page 12

$P_{\text {eff }}=$ Effective reflectance of the sphere wall as defined on page 20. 


\section{DESCRIPTION OF THE EXPERIMENTAL SETUP *}

The equipment consisted of an integrating sphere, a He-Ne continuous wave gas laser as a source, a $10 \mathrm{KW}$ radio frequency generator, a He purification system and a vacuum unit. A two-detector amplifier system was used for the measurement of incident and reflected flux, while the temperature was measured by either a thermocouple or an optical pyrometer.

The sphere was formed by two stainless steel hemispheres, $35 \mathrm{~cm}$ in diameter. All flanges were inert gas welded and had 0-ring grooves for vacuum tight seals. The sphere had four openings, two in each hemisphere. The upper hemisphere contained the entrance port and the detector port. The lower hemisphere carried the ports for sample holder and comparison standard, which could be replaced by a plug to close the port. In addition to these ports, evacuation slots were arranged concentrically around the sample port. A detailed description as well as a theoretical analysis of the integrating sphere is given in Section 2.3.

The sphere was coated with sodium chloride. The coating was applied by spraying a sodium chlorideethyl alcohol suspension. Since sodium chloride had never been used before as an integrating sphere coating, an experimentál program was started to investigate the reflective properties of the sodium chloride coating. The experimental procedures employed and the results obtained are given in Appendix A: The results showed that sodium chloride was indeed a very good choice for an integrating sphere coating.

As mentioned in the introduction, the necessity of a high intensity source rules out any conventional radiation sources such as Globars or tungsten strip lamps. At temperatures around $2000^{\circ} \mathrm{K}$, the signal to background ratio would be so small that it would be extremely difficult to recover the signal with a high enough accuracy. The source chosen was a He-Ne continuous wave gas laser. Three pairs of interchangeable mirrors were provided to permit operation of the laser at $0.6328,1.15$ and $3.39 \mu$ / , respectively. The laser was used in the multimode confocal configuration. The multimode operation has the advantage of high amplitude stability and also increases the power output. The confocal configuration makes alignment of the mirrors less critical [5]. The plasma tube was closed off on each end by a Brewster angle window. These Brewster angle windows polarized the laser beam. The beam of the laser was highly collimated which eliminated the need for any external collimating or focusing optics. The nominal output of the laser was given by the manufacturer as one milliwatt at $0.6328 \mu \mathrm{m}$.

The samples were heated by induction. Electrically conducting samples were placed in a thoria crucible and heated directly. Nonconducting samples, for example metal oxides, were put in tungsten or iridium susceptors. The susceptor, which is heated by induction, then transfers the heat to the sample by conduction and radiation. Figure 11 shows the specimen heater and its location at the sphere. The heater was a pancake shaped induction coil; the innermost turn was soldered to a slotted copper plate which acted as a concentrator. The remaining turns were insulated from the concentrator by a sheet of Teflon. The concentrator was machined to the curvature of the sphere, was coated and thus formed an integral part of the sphere wall. A cooling loop around the edge of the copper plate removed the excess heat. The whole unit was enclosed by a Pyrex cylinder sealed off on both ends by Teflon gaskets. The two flanges separated by the Pyrex cylinder were drawn together by twelve Bakelite bolts.

\section{2/ See Section 5.1.5.}

${ }^{*}$ Certain commercial materials and equipment are identified $\mathrm{n}$ this paper in order to adequately specify the experimental procedure. In no case does such identification imply recommendation or endorsement by the National Bureau of Standards, nor does it imply that the material or equipment identified is necessarily the best available for the purpose. 

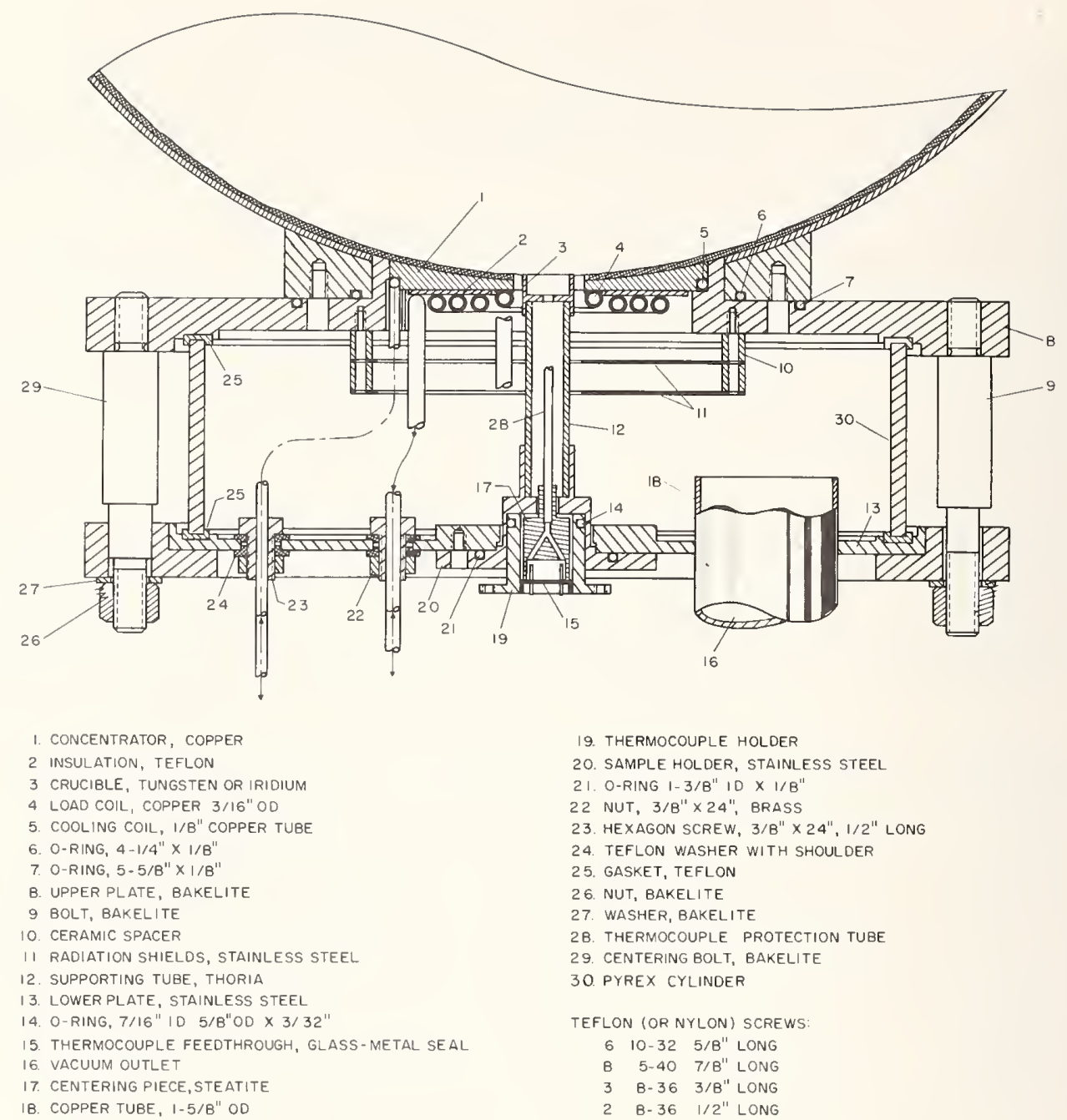

Figure 11. Sample Heater and Sample Holder Assembly

The sample holder assembly, also shown in Figure 11, could be removed by unscrewing three bolts in the lower plate. The whole assembly then slipped through a hole in the lower plate. This made it easy to change samples and to adjust the thermocouple with respect to the sample. The crucible or susceptor was supported by an alumina tube which reduced the conduction of heat to the stainless steel sample holder. Centering the sample in the hole of the concentrator was facilitated by a shoulder on the sample holder that fitted into a hole in the lower plate. This plate in turn fitted into a shoulder on a Bakelite ring, which was positioned with respect to the upper plate by three centering screws spaced $120^{\circ}$ apart around its rim. Thus the sample holder could be removed and replaced without disturbing the alignment of the specimen.

During measurement it was first necessary to precisely position the laser beam onto the sample, and then onto a spot on the sphere wall. Since a visual check of the irradiated area in the infrared was not possible, the use of a micrometer type adjustment was ruled out. Therefore, a flip-flop mirror was devised which, after initial setting, allowed switching of the beam from the sample to the reference spot and vice versa. No additional adjustment was necessary. The mirror has one axis of rotation lying in its front surface and in the principal plane (for definition of the principal plane see Section 2.2). A second axis of rotation is provided perpendicular to the first one. In the two switching positions, 
the mirror mount is held by two magnets, cemented to the base plate, which are resting against steel wedges. The wedges have push-pull adjustments. An exploded view of the mirror mount is shown in Figure 12 .

For measurements at 0.6328 and $1.15 \mu \mathrm{m}$, an uncooled lead sulfide cell with a sensitive area of 10 x $10 \mathrm{~mm}$ was used. For measurements at $3.39 \mu \mathrm{m}$, the uncooled detector was replaced by a liquid nitrogen cooled lead sulfide cell with a sensitive area of $8 \mathrm{x} 8 \mathrm{~mm}$. Cooling the cell to $-196^{\circ} \mathrm{C}$, the temperature of liquid nitrogen, causes a shift of the maximum of specific responsivity $\underline{3}^{\prime}$ from approximately $2 \mu m$ for an uncooled cell to about $3 \mu \mathrm{m}$, and also increases the specific responsivity over its entire useful spectral region. Figure 13, taken from a data sheet of the Eastman Kodak Company, shows the specific responsivity for an uncooled and a cooled lead sulfide cell. Figure 14 is a sketch of the light trapfilter-detector arrangement on the sphere.

The radio frequency generator is operated at $450 \mathrm{KHz}$ and has a maximum power output of $10 \mathrm{KW}$. The power output can be regulated continuously by use of thyratron tubes and a saturable core reactor. The setting of the thyratron tubes is used to limit the maximum power output while the saturable core reactor is used to vary the power from near zero to that preset maximum.

To avoid measurable oxidation of a sample, the air pressure must be held to about $10^{-5}$ torr or less. Therefore, as mentioned earlier, the integrating sphere and the heating unit were built vacuum tight to allow evacuation of the sphere. The vacuum system used consisted mainly of a mechanical pump,

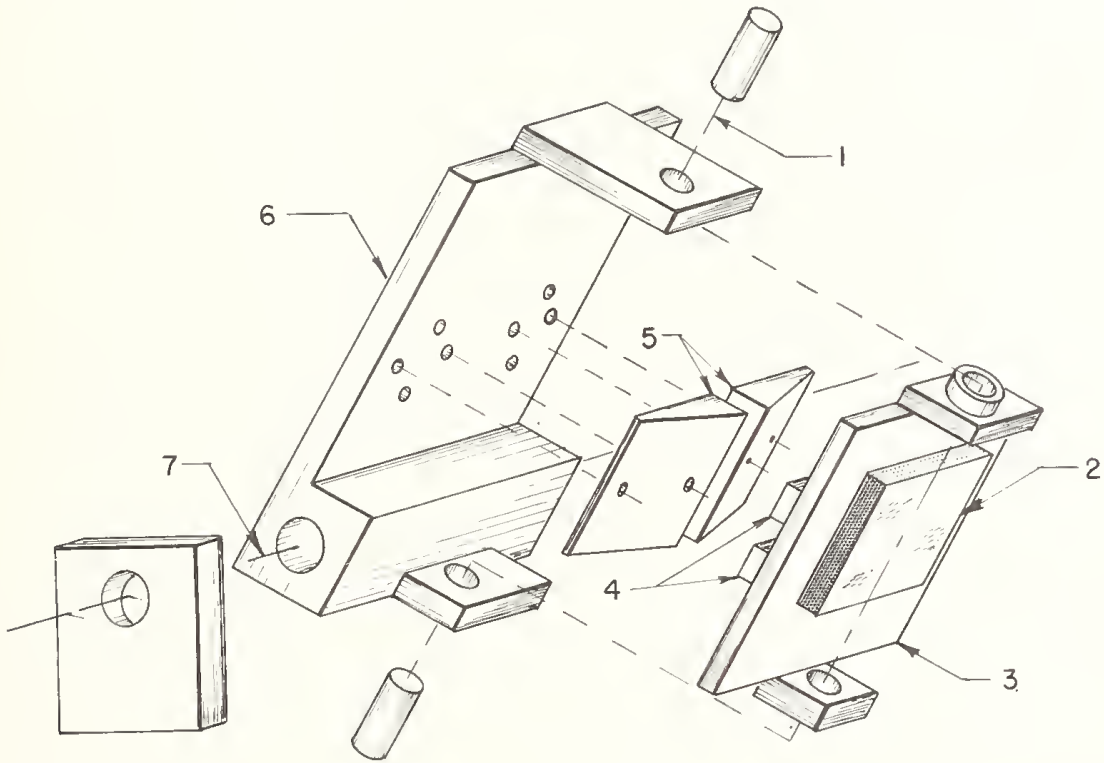

1. First axis of rotation

2. Mirror

3. Plate

4. Mognet

5. Wedge

6. Boseplate

7. Second axis of rotation

Figure 12. Mirror Mount

3/ The specific responsivity is equal to the signal in microvolts produced by a photodetector having one volt of bias applied across the detector and matching load resistor combination when irradiated by a flux density of one microwatt per square centimeter. 


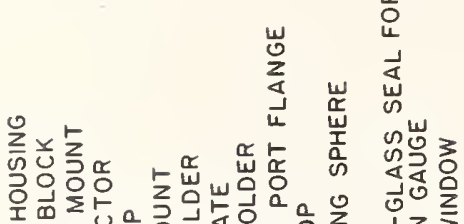

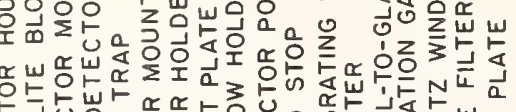

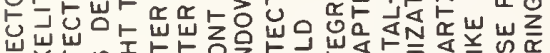

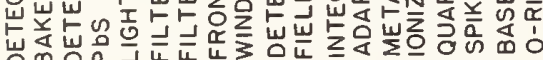
-
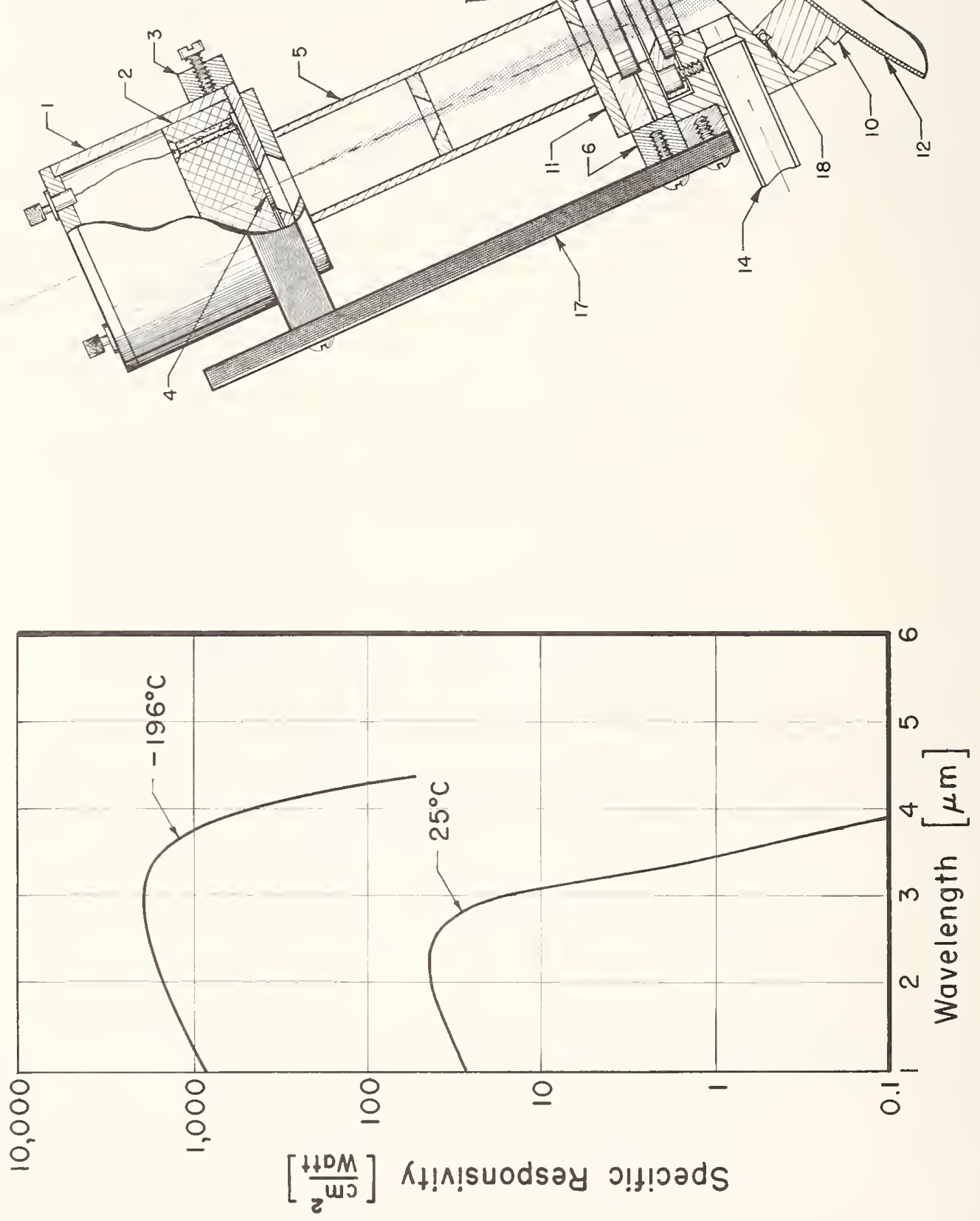

至 
a 6-inch oil diffusion pump, and a cold trap between the diffusion pump and the gate valve to reduce backstreaming of oil vapors into the integrating sphere. The cold trap was cooled by a refrigeration unit. The temperature obtained in the cold trap was approximately $-55^{\circ} \mathrm{C}$. The ultimate pressure of the system was found to be around $1 \times 10^{-7}$ torr.

Measurements at the highest temperatures were made in helium because the vapor pressure of the materials increases rapidly at high temperatures. For example, the vapor pressure of tungsten increases from $10^{-8}$ torr at $2350^{\circ} \mathrm{K}$, to $10^{-7}$ torr at $2500^{\circ} \mathrm{K}$, and becomes $10^{-4}$ torr at $3050^{\circ} \mathrm{K}$. Hence significant evaporation may occur around $2500^{\circ} \mathrm{K}$ at $10^{-6}$ torr which, of course, would ruin the sphere coating. The use of an inert atmosphere at a pressure near atmospheric pressure greatly reduces the contamination of sphere coating and windows from volatilization of the specimen or susceptor, without appreciably increasing the oxidation.

Helium was chosen instead of argon for two reasons. First, helium has an ionization potential of 24.46 volts compared with 15.68 volts for argon, hence is less subject to arcing at the concentrator slot. Secondly, cold traps cooled by liquid nitrogen can be used to remove all condensable impurities from helium without condensing the inert gas itself. The purity of the helium used was given by the supplier as $99.999 \%$. The content of impurities of main concern, namely oxygen and water vapor, was given as less than $2 \mathrm{ppm}$ and $2.4 \mathrm{ppm}$ respectively. The purity was further increased in a purification system which used copper in fine powder form deposited on diatomaceous earth. This material can be bought commercially in the form of pellets under the name BTS catalyst. The method has been described in detail by F. Maak and C. M. Sellars [6]. Figure 15 shows the arrangement of the purification system.

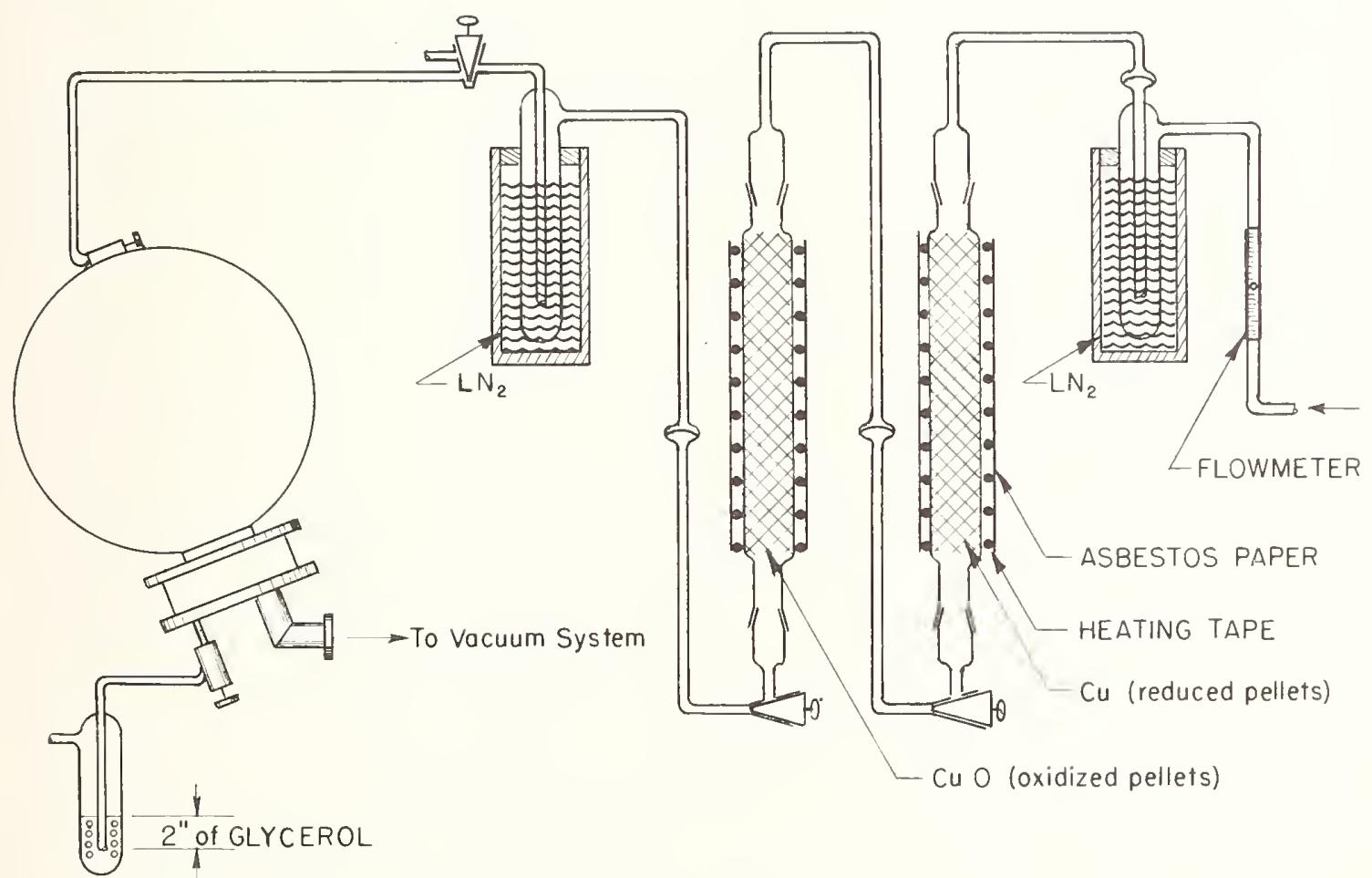

Figure 15. Purification System 
One problem experienced during the early stages of the development was the instability of power output of the laser. Therefore, a two-detector system was used to make the measurement independent of the output level of the laser. Figure 16 shows the signal flow diagram. The 1 aser beam is chopped by a first-surface mirror chopper at $33.9 \mathrm{cps}$. During the first half of each cycle, the beam is incident on the flip-flop mirror above the entrance port and is reflected onto the sample or the sphere wall. During the second half, the beam is reflected by the chopper blade into a small averaging sphere, and the radiance of the averaging sphere wall is measured by the reference detector, whose signal is then fed into a phase sensitive synchronous amplifier. The signal from the detector of the integrating sphere is amplified in an identical amplifier. The output of the two amplifiers is then put into an electronic system whose output is proportional to the ratio of the two signals. The readout device used is an integrating digital voltmeter. With this system, all measurements are made relative to the incident flux, which makes the accuracy independent of the stability of the laser.

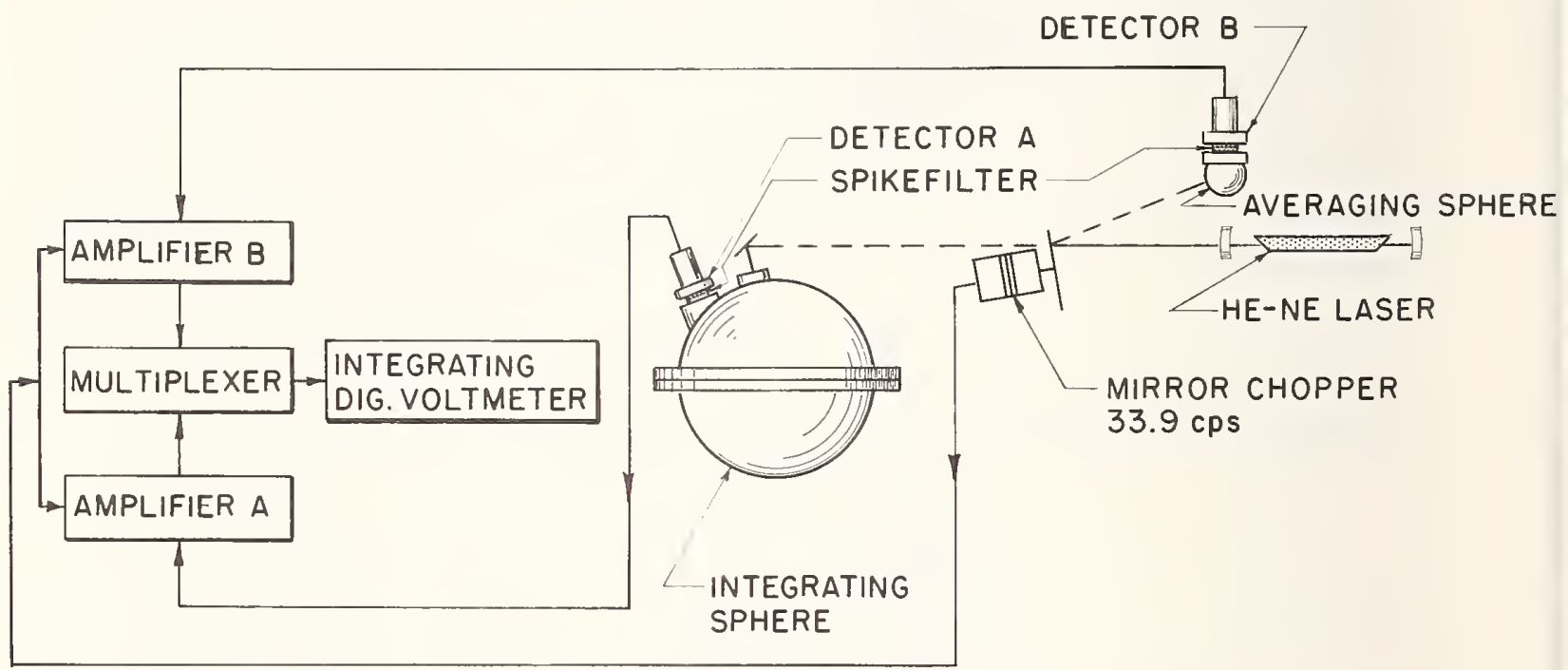

Figure 16. Signal Flow Diagram

4. DESCRIPTION OF THE SAMPLES

Measurements were made on thoria $a^{4}$, graphite $\underline{5}^{\prime}$, and tungste ${ }^{6}$ ' samples at the wavelengths of 0.6328 and $1.15 \mu \mathrm{m}$. Al1 samples were discs $1 / 2$ inch in diameter. The thickness was $1 / 4$ inch for the thoria sample and $1 / 8$ inch for the others.

4/ The thoria samples were obtained from the Laboratory Equipment Corporation.

5/ The graphite used was grade UT6 procured from U1tra Carbon Company, Bay City, Michigan.

6/ The supplier of the tungsten was cleveland Tungsten, Incorporated. 
A chemical analysis of the thoria specimens supplied by the vendor showed in percent by weight $99 \mathrm{ThO}_{2}, 0.05 \mathrm{SiO}_{2}, 0.02 \mathrm{MgO}$ and a trace of $\mathrm{CaO}$. Also present were trace amounts of the oxides of phosphorus, iron and rare earths as well as a minor amount of alkali oxides. The porosity of the specimen was $13 \%$ by volume.

The sum of the impurities in the graphite was given as 10 to 50 ppm consisting mainly of Mg, Fe, and $\mathrm{Si}$. The material had an apparent porosity of $21 \%$ and a maximum grain size of 0.003 in. The samples were smoothed by grinding them with fine grades of metallographic polishing paper. The final finish was obtained by hand polishing on lens paper.

The tungsten samples were made from arc cast material. The surface of the samples was ground and optically polished. The samples were then slowly heated in hydrogen to $2000^{\circ} \mathrm{K}$ and kept at that temperature for 15 minutes, then cooled slowly.

Since the reflectance of opaque materials is very much a function of the surface texture, the surface conditions were examined before and after the measurements. In the case of the tungsten sample, the surface was characterized three times, namely before heat treatment, after heat treatment and finally after the integrating sphere measurements. Three methods were used for characterization. First, the surface roughness was measured by using a stylus instrument. Secondly, for the graphite and tungsten samples, microinterferograms were taken. These interferograms give a better overall description of the surface than the traces of the stylus instrument, because the traces give only one cross section of the sample, which may or may not be characteristic of the surface. The third method was the use of photomicrographs. Although no quantitative information can be extracted from the photographs, changes in the crystal structure will clearly show up.

\subsection{Thoria}

The first material measured was thoria. Figure 17 and 18 show copies of the original stylus traces (profiles) of the thoria sample before the measurements were taken. The two traces are two different cross sections of the sample. The arithmetic average (AA) roughness, which represents the

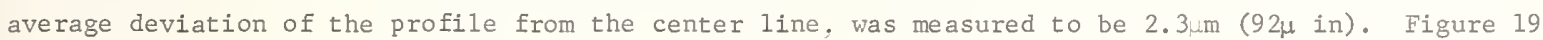
shows a trace of the same sample after it had been subjected to four heating cycles in the integrating sphere. The arithmetic average roughness was measured as $2.4 \mu \mathrm{m}$ ( $95 \mu$ in). No changes in structure can be deduced from these figures. Although photomicrographs were also made from the sample, they are not presented because they do not reveal the structure of the thoria. Thoria is a partially translucent material. Therefore, the incident light is reflected from within a finite layer of the sample and the surface of the sample is not readily visible.

\subsection{Graphite}

The next sample measured was the graphite sample. Figure 20 gives a stylus trace of the graphite sample. There existed definitely a preferred orientation of the scratches in the surface and the trace was taken perpendicular to the main direction of the scratches. The arithmetic average roughness was measured as $0.29 u$ m ( $11 \mu \mathrm{in.)}$. However, it should be noted that even with a 1 oad of $30 \mathrm{mg}$, the stylus made a visible scratch, which means that the stylus was partially cutting through the material. Therefore, the actual roughness could be higher than that indicated by the measurements. Figure 21 shows a trace of the same sample after it had undergone four heating cycles. The AA roughness was measured as $0.26 \mu \mathrm{m}(10 \mu \mathrm{in})$. This indicates that no measurable change in surface roughness had occurred. Figures 22 and 23 show microinterferograms of the graphite sample before and after heating, respectively. No 


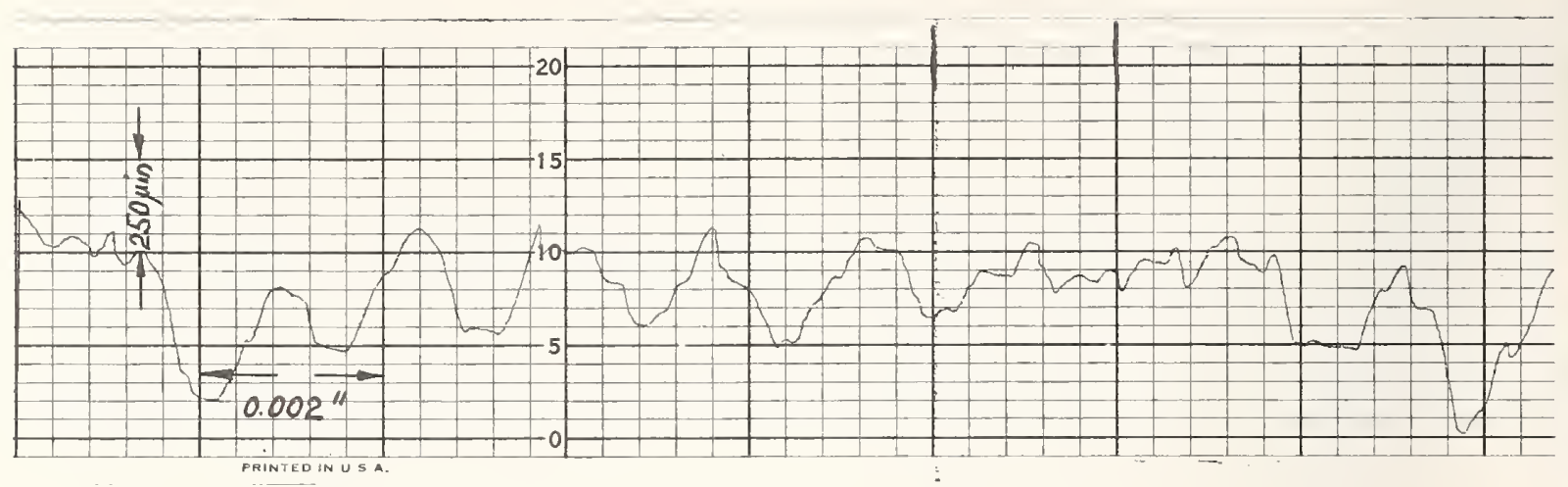

Figure 17. Stylus Trace of Thoria Sample Before Heating

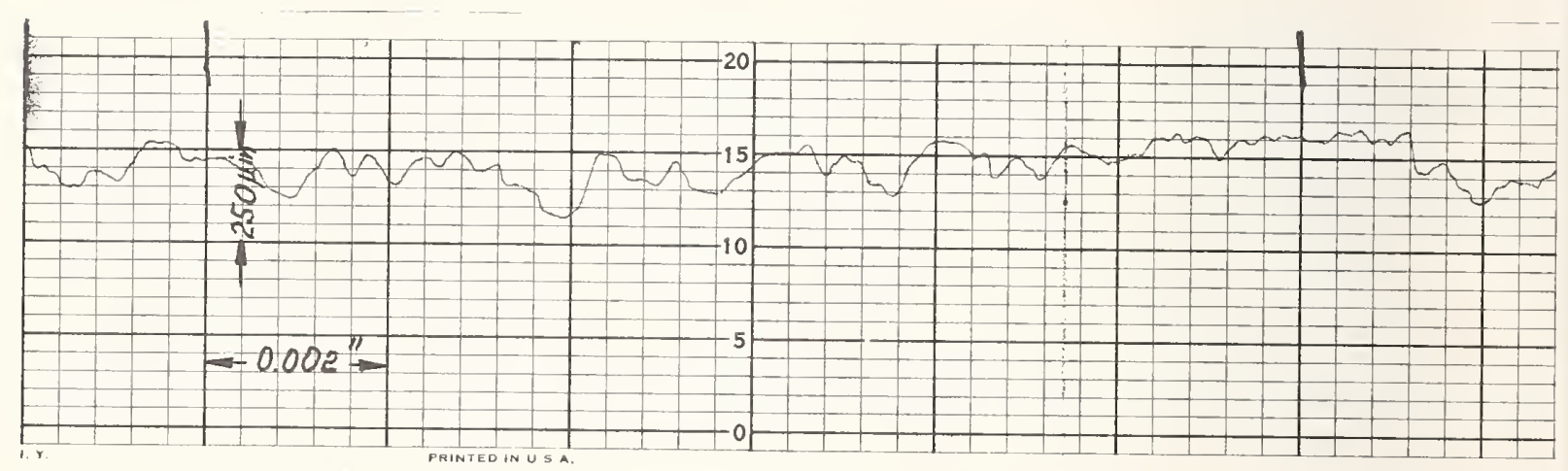

Figure 18. Same as Figure 17 Except Different Cross Section

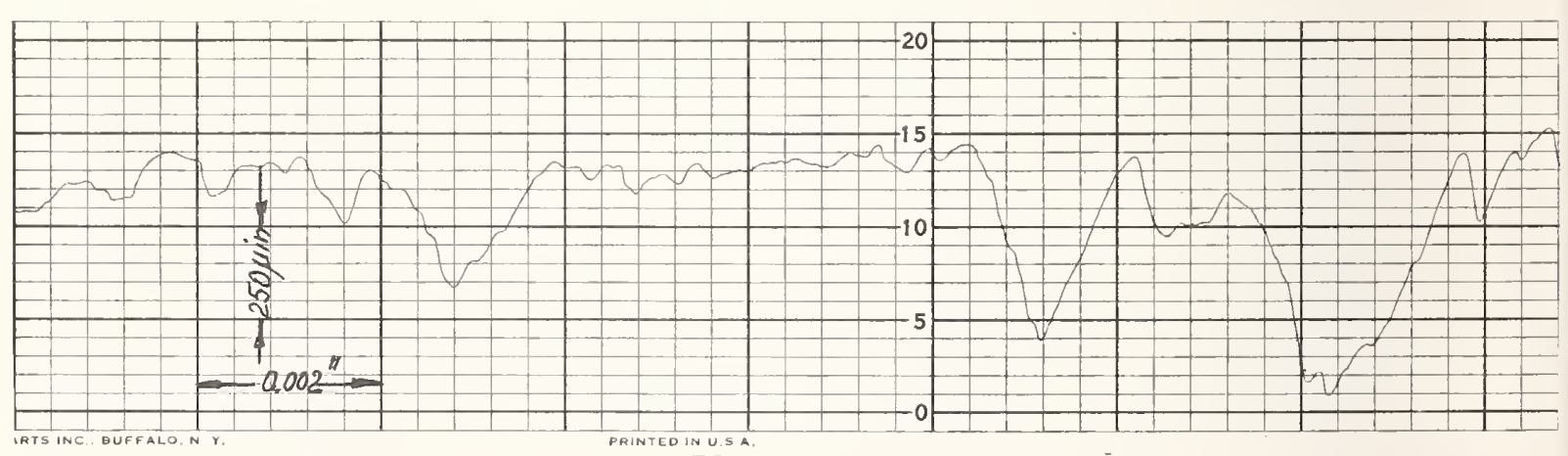

Figure 19. Stylus Trace of Thoria Sample After Four Heating Cycles 


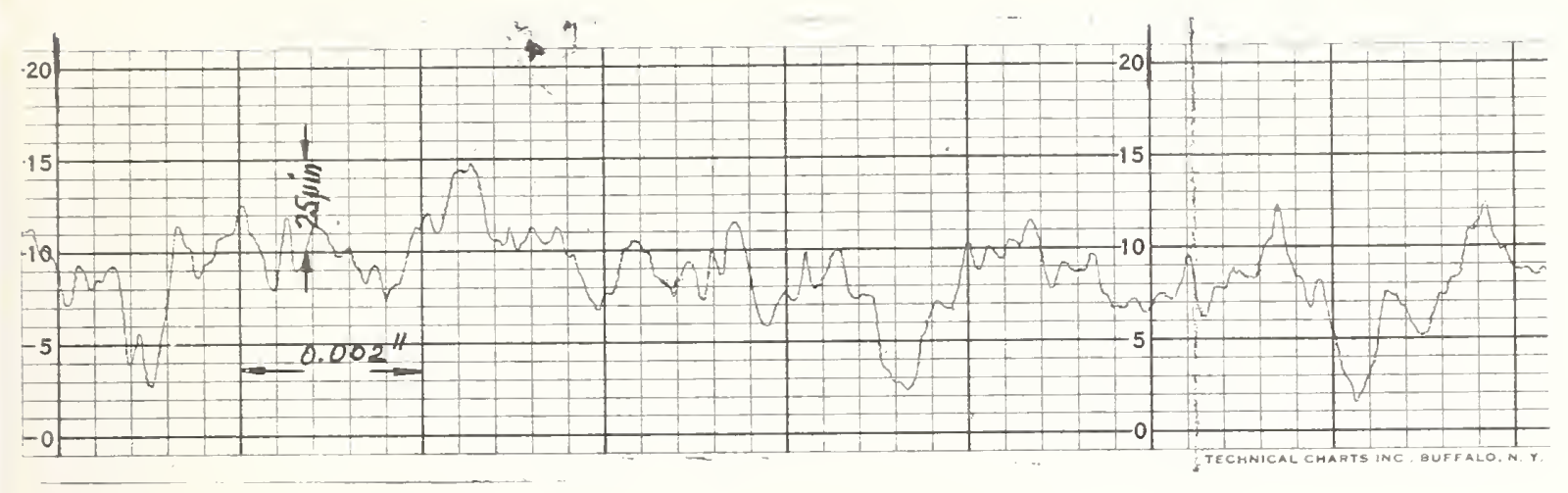

Figure 20. Stylus Trace of Graphite Sample Before Heating

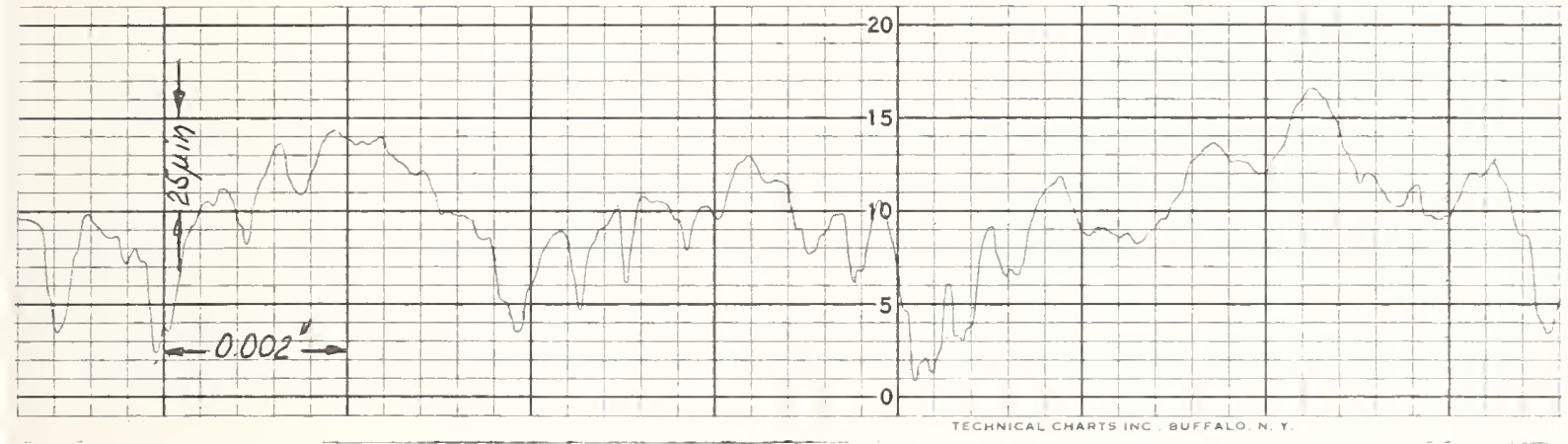

Figure 21. Same as Figure 20 After Four Heating Cycles

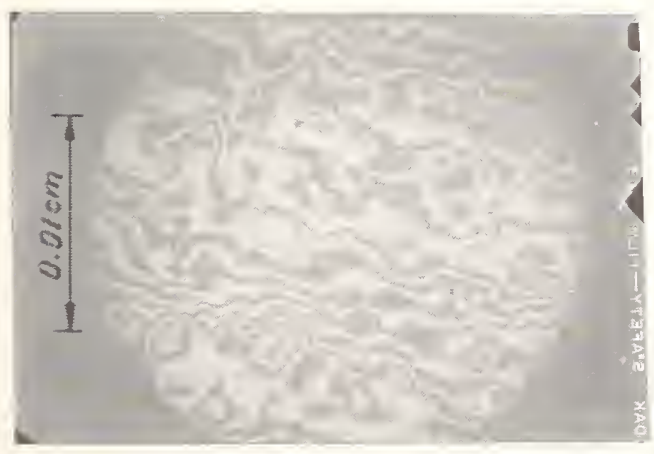

Figure 22. Microinterferogram of Graphite Sample Before Heating

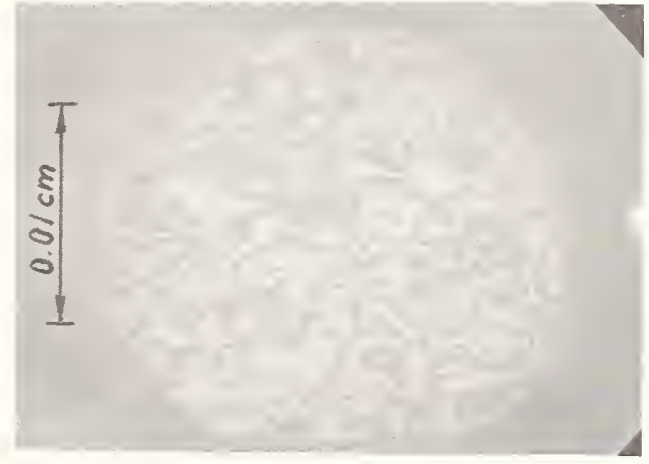

Figure 23. Same as Figure 22 After Four Heating Cycles 
changes in surface structure are obvious. Figure 24 is a photomicrograph of the graphite sample and Figure 25 shows the surface after the sample had undergone four heating cycles. No change in the surface structure can be detected. The surface lacks any sharp contours or scratches as can be expected from a soft material like graphite.

\subsection{Tungsten}

The last material measured was a sample of tungsten. Figure 26, 27, and 28 are copies of stylus traces taken from the tungsten sample after polishing, after heat treatment as described above, and after three heating cycles. The measured AA roughness was $0.025,0.02$, and $0.17 \mu \mathrm{m}$ ( 1 , 0.9 , and $6.8 \mu \mathrm{in}$ ) respective1y. These results show that, although the heat treatment had not effected the surface roughness measurably, a marked increase in roughness had occurred during the three heating cycles in the integrating sphere. Figures 29, 30, and 31 are microinterferograms of the tungsten sample again taken after polishing, after heat treatment and the last one after three heating cycles up to $2150^{\circ} \mathrm{K}$ under vacuum. Figure 29 shows that the sample was indeed quite flat. Figure 30 reveals that the surface texture had undergone a change as indicated by breaks in the interference fringes. Figure 31 shows that the surface had changed drastically during the reflectance measurements as seen in the stylus traces.

Figures 32, 33 and 34 show photomicrographs of the same sample taken at the same three stages. Figure 32 shows no surface structure of any kind except for scratches due to the polishing procedure. The next figure indicates clearly that the surface had undergone a definite change in structure. It seems that a recrystallization process had at least been started during the heating. Comparing Figure 34 with the preceding photograph, we see that the surface had again changed considerably. The surface structure was much finer. This change was believed to be caused by thermal etching so that the individual crystal planes showed within each tungsten crystal. This process could have been intensified by trace amounts of oxygen and water vapor in the integrating sphere. All oxides formed have a much higher vapor pressure than the pure metal and will evaporate rapidly at the high temperatures. As we will see in the next chapter, these changes of the surface structure lead to definite changes in the reflectance.

Although it could not be seen on the photomicrographs, the thoria sample had also changed during the four heating cycles. The change manifested itself in a change of color. The sample after four heating cycles appeared almost white when compared with the yellowish color of the unheated sample. Again this change expressed itself quite clearly in a change of reflectance as is shown in the next section. This change could have been produced either by volatilization of impurities on heating in vacuum, some change in crystal structure, or to a change in state of impurity constituents. The exact mechanism responsible for the observed change was not established. 


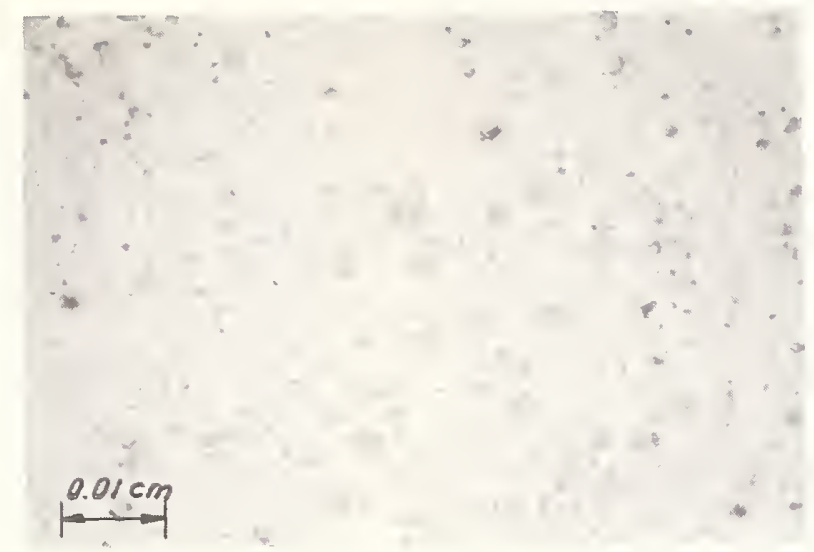

Figure 24. Photomicrograph of Graphite Sample

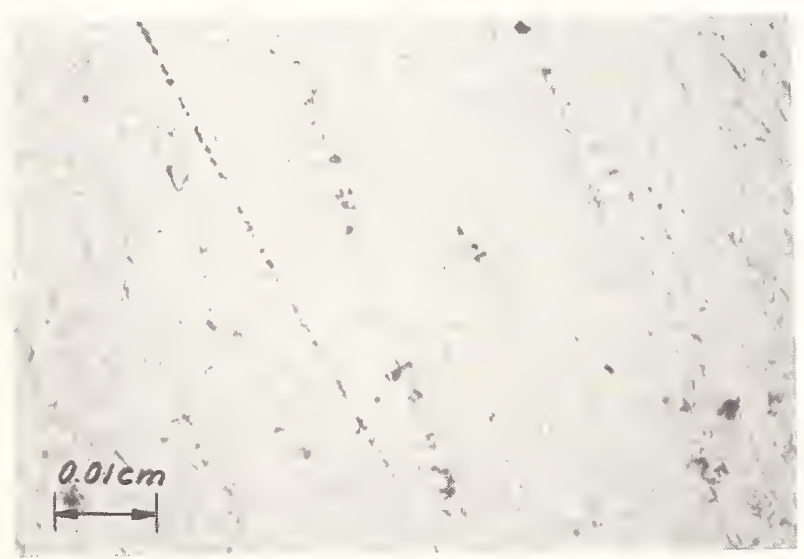

Figure 25. Same as Figure 24 After Four Heating Cycles

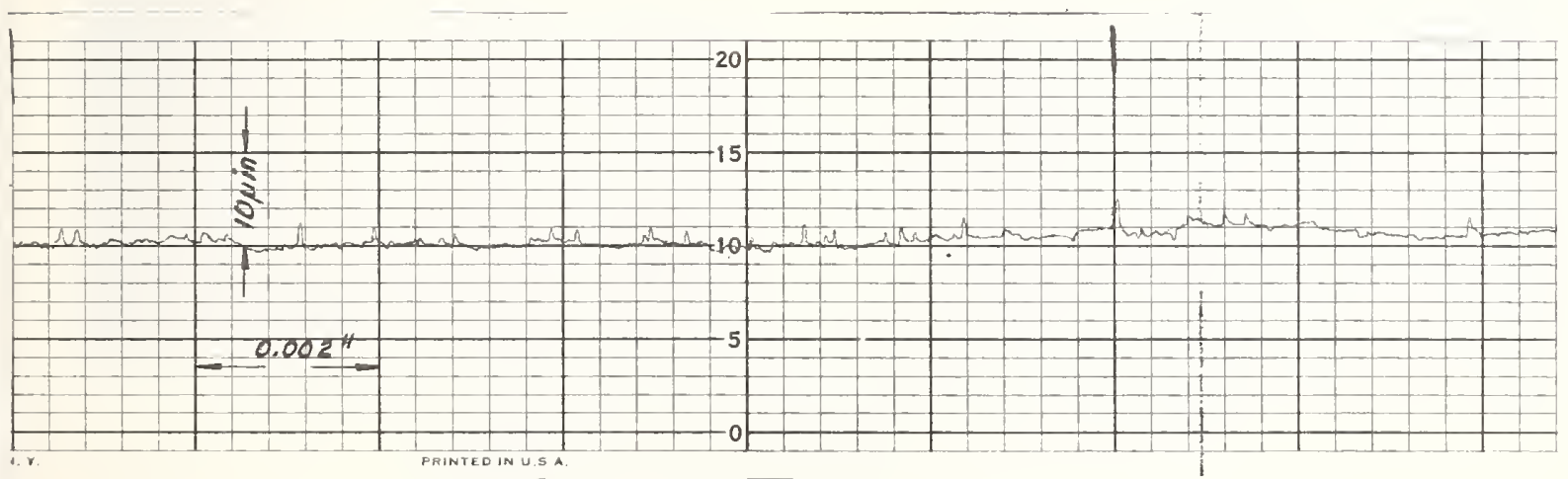

Figure 26. Stylus Trace of Tungsten Sample After Polishing Procedure 


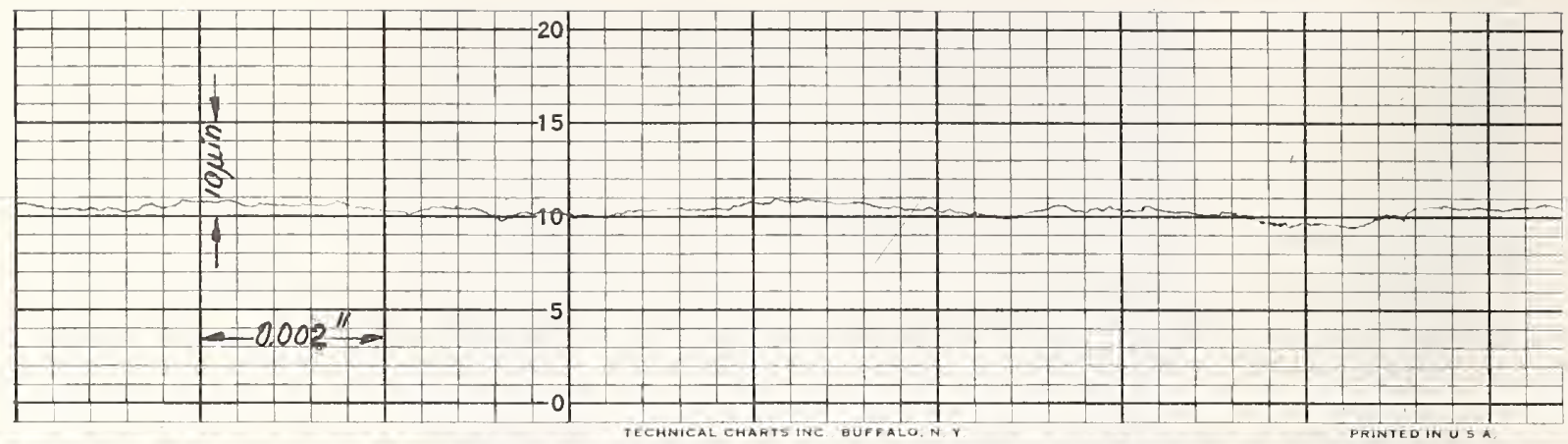

Figure 27. Same as Figure 26 After Heat Treatment

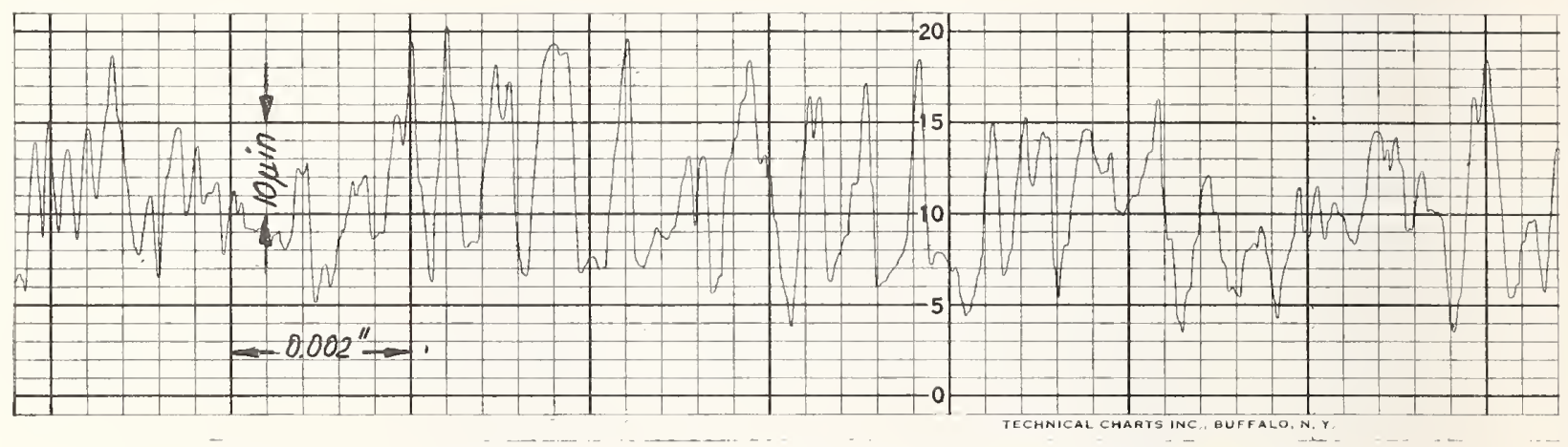

Figure 28. Same as Figure 26 After Heat Treatment and Three Heating Cycles

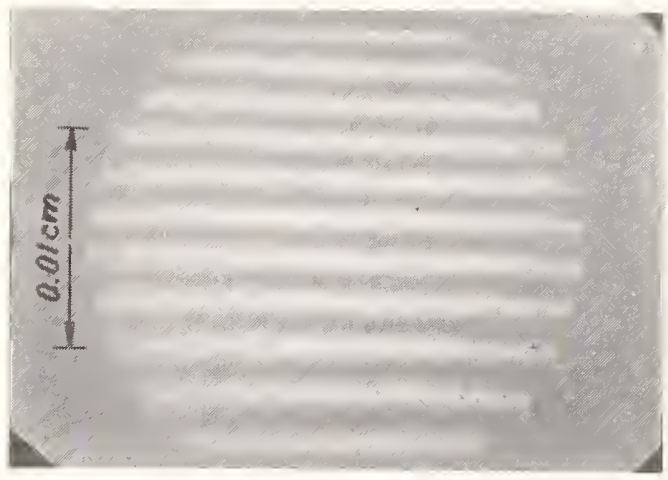

Figure 29. Microinterferogram of Tungsten Sample After Polishing.

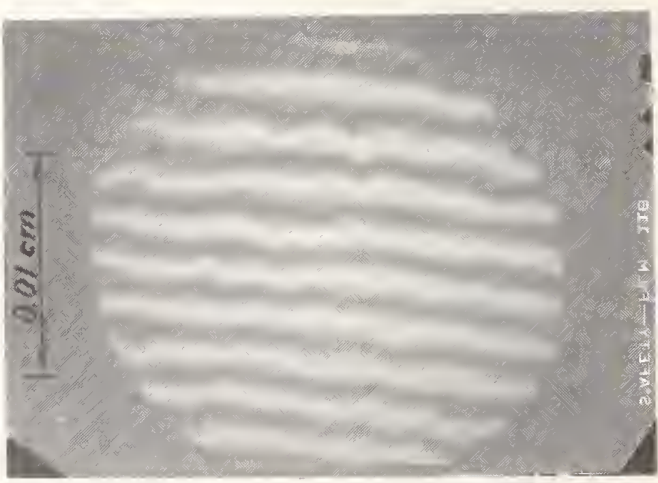

Figure 30. Same as 29 After Heat Treatment. 


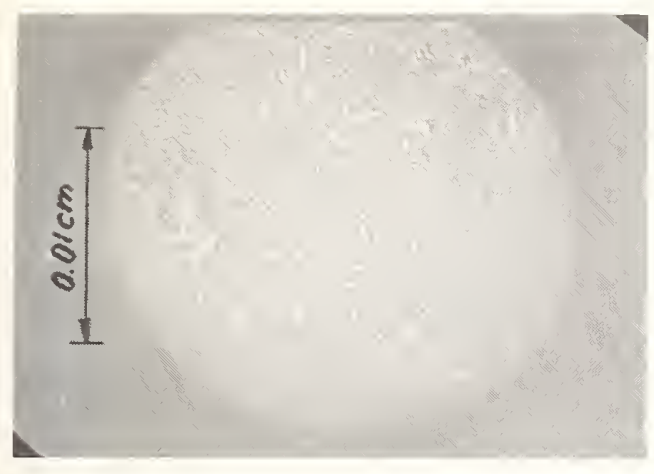

Figure 31. Same as 29 After Heat Treatment and 3 Heating Cycles.

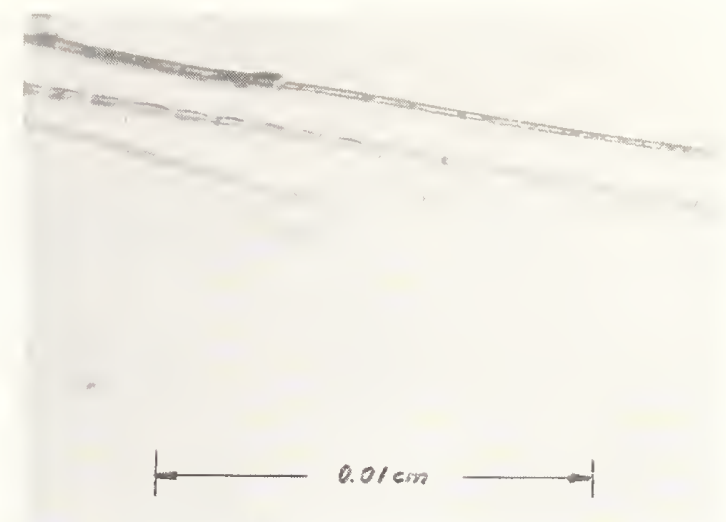

Figure 32. Photomicrograph of Tungsten Sample After Polishing

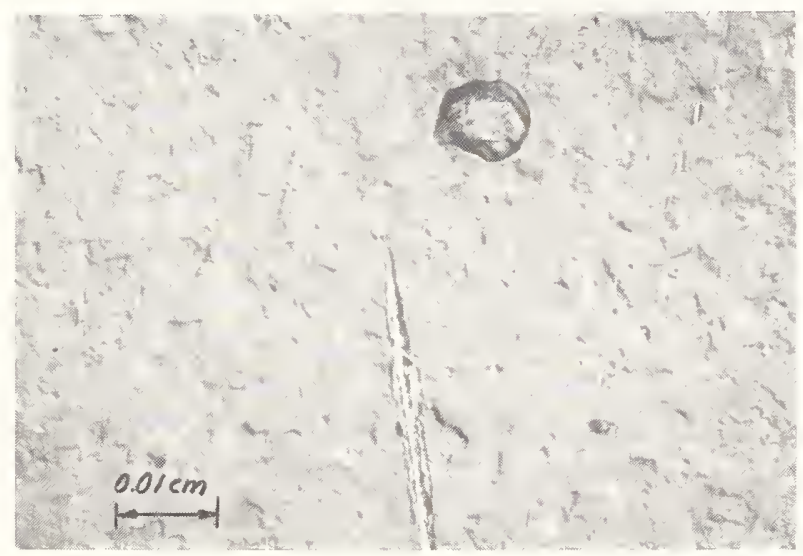

Figure 33. Same as Figure 32 After Heat Treatment

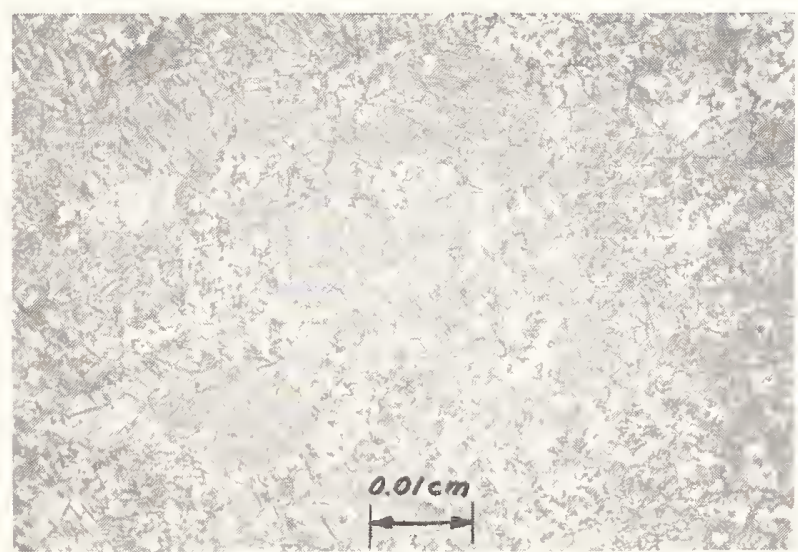

Figure 34. Same as Figure 32 After Heat Treatment and Three Heating Cycles. 


\section{RESULTS AND CONCLUSIONS}

\subsection{Experimental Verification of the Error Analysis}

Before presenting and discussing the high temperature reflectance data, it seems desirable at this point to introduce an experimental verification of the error analysis.

In Section 2, a theoretical analysis was undertaken to compute the systematic errors present in the reflectance measurements made with this integrating sphere. The results were presented in Tables I, II, III and IV and also in Figure 10. That analysis, which assumed either a perfectly specular or a perfectly diffuse sample, was based on certain assumptions. Therefore, the results of this experimental analysis will show to what degree those assumptions for an ideal integrating sphere are validated by the real integrating sphere. Of the two cases analysed, the case of the perfectly specular sample was chosen to be tested experimentally for two reasons. First the reflectance of the specular sample can be measured more accurately than a diffuse sample by an independent technique. Secondly, the perfectly specular sample can be approximated to a very high degree by evaporating a metal film onto a highly polished quartz substrate.

5.1.1. Preparation of the Mirrors: Mirrors of the same size as the samples used in the integrating sphere were prepared at the optical shop of the National Bureau of Standards. The quartz substrates were polished by a so-called super polishing technique. The pitch polisher was completely immersed in water and the polishing agent was only added once. The flatness of the discs was better than $1 / 20$ of a wavelength of the sodium D Line. After the polishing was completed, the substrates were cleaned carefully by washing them with solvents, detergents, boiling water, and finally with doubly-distilled acetone. Then the quartz discs were inserted in the vacuum chamber and cleaned by a glow discharge for about 20 minutes. Two sets of mirrors were prepared. For one set $99.999 \%$ pure aluminum was evaporated at a pressure of $1 \times 10^{-6}$ torr and then a shutter between the filament and the discs was opened for approximately 5 seconds. For the second set, gold of the same purity was used. The deposition and measurement techniques for thin films has been well developed. An excellent source of information on al1 details concerning thin films is Bennett and Bennett [7] with a complete list of references.

5.1.2. Description of the Specular Reflectometer: In order to calibrate the mirrors, it was necessary to use a reflectometer which was capable of measuring the reflectance to a much higher accuracy and precision than could be attained with the integrating sphere. Therefore, a specular reflectometer was designed and built based on the well known multiple reflection technique. A sophisticated specular reflectometer employing this principle is also described in the reference just cited.

The reflectometer used in this experiment consisted of an aluminum disc into which three rectangular slots had been milled. Care was taken to machine the slots parallel and square as closely as possible. Three blocks of brass served as mirror holders. Holes were drilled into the brass blocks of a slightly larger diameter than that of the mirrors. The alignment of the mirrors was achieved by gently pressing the front surface of the mirrors against the diaphragm attached to the brass block. Figure 35 shows a cross section of a mirror holder. Tightening the adjustment screw by hand was more than sufficient to insure that the mirror's front surface was properly resting against the diaphragm. 


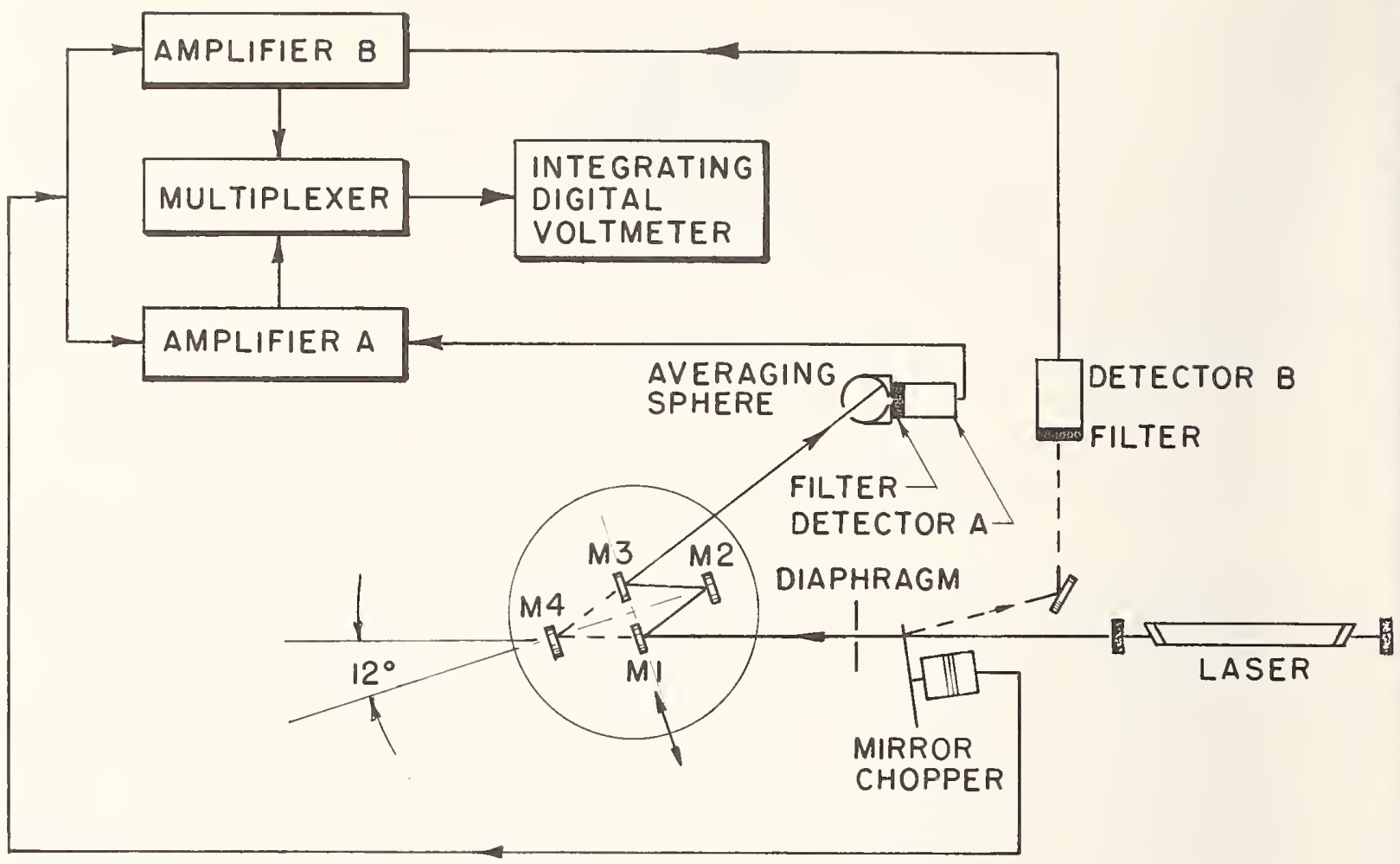

Figure 36. Specular Reflectometer

The same technique as applied for the integrating sphere reflectometer measurements was used with the specular reflectometer to compensate for power-output fluctuations of the 1 aser.

5.1.3. Calibration of the Mirrors: The measurement procedure itself was quite simple. Four mirrors of the same set were inserted into the holders and all components aligned. Then the center mirror holder was moved into the path of the beam and the signal $\mathrm{S}_{1}$ was read from an integrating digital voltmeter. About twenty consecutive readings were taken. Then the beam was blocked behind the diaphragm

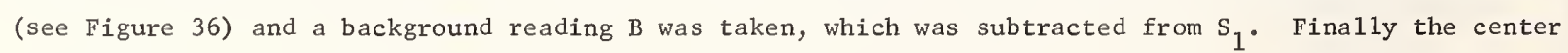
mirror holder was removed from the path of the beam which resulted in a signal $\mathrm{S}_{2}$. The functional relationship between the reflectances of the mirrors and the measured signals is then given by equation (27): 


$$
\frac{S_{1}-B}{S_{2}-B}=\frac{\rho_{1} \rho_{2} \rho_{3}}{\rho_{4}}
$$

Assuming that the reflectance of all four mirrors is the same and equal to $\rho$, then $\rho$ is given by equation (28):

$$
p=\sqrt{\frac{S_{1}-B}{S_{2}-B}}
$$

Since the mirrors in one set were prepared in exactly the same way and coated at the same time, this assumption seemed justified. However, in order to check this assumption, p was calculated frorn the measurements with two different groupings of the mirrors as indicated in equations (29) and (30):

$$
\begin{aligned}
& \rho_{3}=\sqrt{\frac{\rho_{1} \rho_{2}{ }^{p}}{\rho_{4}}} \\
& \rho_{b}=\sqrt{\frac{\rho_{4}{ }_{2} p_{3}}{\rho_{1}}}
\end{aligned}
$$

As noted in Section 3, the laser beam is plane polarized and the orientation of the plane of polarization is determined by the orientation of the Brewster angle windows. As seen from reference [8], page 20, the reflectance for the component of the radiation whose electric field vector is paralle1 to the plane of incidence goes to zero for a nonconductor when the angle of incidence is equal to the Brewster angle. This component, therefore, is transmitted unattenuated and gets amplified by stimulated emission. In other words, the plane of polarization of the laser beam is given by the plane of incidence formed by the normal to the window and the incident ray. This property of the laser made it possible to measure the reflectance of the mirrors for the two components of polarization. First the laser was aligned such that the plane of polarization was parallel to the plane of incidence of the mirrors. This plane is called the $\mathrm{p}-\mathrm{plane}$; and the reflectance for incident flux polarized in the pplane is, therefore, indicated by using the subscript p. After all measurements of $p_{p}$ were completed, the laser cavity was rotated in its holders by $90^{\circ}$, the laser realigned and all measurements repeated. The plane which contains the incident ray and is perpendicular to the p-plane is called the s-plane and the respective reflectance for incident flux polarized in the s-plane is then symbolized by $P_{S}{ }^{*}$ Again from reference [8] on page 21, we see that $p_{p}$ should be less than $p_{s}$ for all angles of incidence except for $\theta=0^{\circ}$ and $\theta=90^{\circ}$ at which $\rho_{S}=\rho_{P}$. If the optical constants $n$ and $k$ are known, then $\rho_{p}$ and $\rho_{S}$ can be calculated from Fresnel's equations as given in reference [9] on page 63. Although optical constants of aluminum and gold have been published [10], this approach was not used because the reflectance of metal films depends greatly on the deposition technique.

5.1.4. Results of Specular Measurements: The results of the measurements with the specular reflectometer are given in Table $\mathrm{V}$ for $0.6328 \mu \mathrm{m}$ and in Table VI for $1.15 \mu \mathrm{m}$. The symbol $\sigma$ for the standard deviation is defined as 


$$
\sigma^{\varepsilon}=\frac{\stackrel{i}{N}_{1}\left(\rho_{i}-\overline{\hat{\rho}}\right)^{2}}{N-1}
$$

where $N$ is the number of measurements taken and $\bar{p}$ is the average reflectance as given by equation

$$
\bar{\rho}=\frac{\sum_{i}^{N} \rho_{i}}{N}
$$

The subscript $a$ or $b$ indicates the grouping of the mirrors as defined in equation (29) and (30). The subscript $s$ or $p$ gives the orientation of the plane of polarization as discussed above. The minimum number of measurements from which the average reflectance and the standard deviation were calculated was seven. If scatter in the data became larger, then the number of measurements was increased (the maximum number used was seventeen) to keep the standard deviation below $0.1 \%$. As we can see from the tables, the only significant difference in reflectance using two different groupings of mirrors, as defined in equations (29) and (30), could be found for the gold mirrors at $0.6328 \mu \mathrm{m}$. The difference measured between $\rho_{z, p}$ and $\rho_{b, p}$ was approximately 0.001 . At $1.15 \mu \mathrm{m}$, the measured difference between $\rho_{\mathrm{S}}$ and $\rho_{\mathrm{P}}$ was essentially within the standard deviation of the measurement itself. Therefore, the two reflectances were only measured for one grouping of mirrors. Only the measurement of $p_{p}$ was repeated with the second grouping. As seen from Table VI, the differences in one type of reflectance between the two groupings are well within the standard deviations of the measurement. The standard deviation of the measurements was between 0.00023 and 0.0009 . The average standard deviation was 0.00041 , which is less than $0.05 \%$. The error in the measurement was certainly not more than $0.1 \%$. This shows that the reflectance of the mirrors was known with an accuracy which made it possible to use them as standards in the integrating sphere.

\section{TABLE V}

REFLECTANCE OF MIRRORS AS MEASURED BY THE SPECULAR REFLECTOMETER AT $0.6328 \mu \mathrm{m}$

$\begin{array}{ccccccccc} & \bar{\rho}_{\mathrm{a}, \mathrm{p}} & \sigma_{\mathrm{a}, \mathrm{p}} & \bar{\rho}_{\mathrm{a}, \mathrm{s}} & \sigma_{\mathrm{a}, \mathrm{s}} & \bar{\rho}_{\mathrm{b}, \mathrm{P}} & \sigma_{\mathrm{b}, \mathrm{P}} & \bar{\rho}_{\mathrm{b}, \mathrm{s}} & \sigma_{\mathrm{b}, \mathrm{s}} \\ \text { gold } & 0.9355 & 0.00055 & 0.9372 & 0.00089 & 0.9365 & 0.00056 & 0.9380 & 0.00035 \\ \text { aluminun } & 0.9030 & 0.00034 & 0.9051 & 0.00067 & 0.9031 & 0.00029 & 0.9054 & 0.00036\end{array}$

TABLE VI

REFLECTANCE OF MIRRORS AS MEASURED BY THE SPECULAR REFLECTOMETER AT $1.15 \mu \mathrm{m}$

$\begin{array}{lcccccccc} & \bar{\rho}_{\mathrm{a}, \mathrm{p}} & \sigma_{\mathrm{a}, \mathrm{p}} & \bar{\rho}_{\mathrm{a}, \mathrm{s}} & \sigma_{\mathrm{a}, \mathrm{s}} & \bar{\rho}_{\mathrm{b}, \mathrm{p}} & \sigma_{\mathrm{b}, \mathrm{p}} & \bar{\rho}_{\mathrm{b}, \mathrm{s}} & \sigma_{\mathrm{b}, \mathrm{s}} \\ \text { gold } & 0.9819 & 0.0003 & 0.9823 & 0.00038 & 0.9823 & 0.00029 & & \\ \text { a luminun } & 0.9600 & 0.00023 & & & 0.9601 & 0.00023 & 0.9605 & 0.00031\end{array}$


5.1.5. Preparation of the Integrating Sphere: Before the reflectance measurements could be repeated in the integrating sphere, the coating had to be replaced because it had been severly damaged during initial testing of the heater. Dielectric breakdown had occurred several times resulting in evaporation of stainless steel where the arc had struck the flange surrounding the heater plate. Since the sodium chloride coating had been mainly developed for use at $3.39 \mu \mathrm{m}$ and since measurements at $3.39 \mu \mathrm{m}$ had to be postponed, the sodium chloride coating was replaced by a barium sulfate coating.

Barium sulfate can be applied very easily by hand pressing. First a thin layer of $\mathrm{BaSO}_{4}$ powder was pressed firmly onto the sphere wall until a shell had been formed. A ring made out of Plexiglas was clamped concentrically on the flange which served to connect the two hemispheres. The inside diameter of this ring was smaller than the inside diameter of the sphere by twice the thickness of the coating. This ring made it possible to build up a firm edge, so that after joining the two hemispheres hardly any crack was visible. After the first layer of powder was pressed onto the sphere wa11, more and more powder was added by pressing it onto the last layer until a thickness of about $1 / 8$ inch was obtained. After the layer had been built up, the coating was smoothed by a glass disc ground to the curvature of the sphere until no marks could be seen. After finishing the coating, the openings in the sphere were carefully cut out with a knife and finally the loose powder was removed by turning the sphere over and brushing with a soft camel hair brush. To coat the concentrator plate, a piece of brass tubing of the right dimensions was recessed on one end such that the inside diameter of the recessed part of the tube was equal to the outside diameter of the plate. The distance between the upper edge of the tube and the upper edge of the plate was made equal to the desired thickness of the coating. The coating procedure for the concentrator plate was the same as for the integrating sphere.

5.1.6. Measurements of the Mirrors in the Integrating Sphere: After the sphere had been set up and all components aligned, the reflectance measurements on the mirrors were repeated. The plane of polarization of the beam was parallel to the plane of the table. Thus the angle between the plane of polarization and plane of incidence of the flip-flop mirror was the same in the two positions of the mirror. This is necessary because otherwise the reflectance of the mirror for the sample position and the reference position would be different, which might result in a large error. This means that the main component of polarization with respect to the sample was in the p-plane.

During the measurements with the specular reflectometer, the mirrors in each set were numbered from one to four. Of each set, mirror 2 and mirror 3 were selected for reflectance measurements. Table VII gives the reflectance of the mirrors as measured with the integrating sphere at 0.6328 and $1.15 \mu \mathrm{m}$. Each value presented is the arithmetic average of seven consecutive measurements. Each single measurement itself was obtained by averaging at least ten successive readings from the integrating digital voltmeter for each measured signal. The standard deviation $\sigma$ is again defined by equations (31) and (32). The symbols $\bar{p}_{2}$ and $\bar{p}_{3}$ stand for the average reflectance of mirror 2 or mirror 3 of any set.

TABLE VII

REFLECTANCE OF MIRRORS AS MEASURID IN THE INTEGRATING SPHERE REFLECTOMETER

\begin{tabular}{lcccccccc}
\hline & \multicolumn{4}{c}{ Gold } & \multicolumn{5}{c}{ Aluminum } \\
\hline & $\bar{\rho}_{2}$ & $\sigma_{2}$ & $\bar{\rho}_{3}$ & $\sigma_{3}$ & $\bar{\rho}_{2}$ & $\sigma_{2}$ & $\bar{\rho}_{3}$ & $\sigma_{3}$ \\
\hline $0.6328 \mu \mathrm{m}$ & 0.9438 & 0.00056 & 0.9430 & 0.00102 & 0.9098 & 0.00118 & 0.9110 & 0.00113 \\
$1.15 \mu \mathrm{m}$ & & & 0.9845 & 0.00113 & & & 0.9630 & 0.00178 \\
\hline
\end{tabular}


No measurements were made for mirror 2 at $1.15 \mu \mathrm{m}$, because the measurements with the specular reflectometer had shown that the variation of reflectance between two groupings of each set were within the standard deviation of the measurement, which was smaller than the standard deviation associated with the integrating sphere measurements. The standard deviations of the integrating sphere measurements ranged from 0.00056 to 0.00178 , with the average being 0.00113 which is only about three times larger than the average standard deviation of the specular reflectometer measurements. This again shows the high precision inherent in this integrating sphere reflectometer. As will be shown later in this Section, the precision can be further improved by using a laser with a higher power output.

5.1.7. Results of the Experimental Evaluation: The systematic error in the integrating sphere measurements can be computed from a comparison of the results obtained with the specular reflectometer and integrating sphere on the same mirror specimens. The measurements with the specular reflectometer should be completely free from any systematic error, and the random error (standard deviation) should be smal1.

In Section 2 the relative error was expressed by equation (17)

$$
E=\frac{\rho_{\text {correct }-P_{\text {measured }}}}{P_{\text {correct }}} \text {. }
$$

Substituting $\left(\bar{p}_{\mathrm{a}, \mathrm{p}}+\bar{\rho}_{\mathrm{b}, \mathrm{p}}\right) / 2$, obtained from Tables $\mathrm{V}$ and $V I$, for the correct value and $\left(\bar{\rho}_{2}+\bar{\rho}_{3}\right) / 2$, computed from Table VII, for the measured reflectance the errors were calculated. These errors are presented in Table VIII.

TABLE VIII

ERRORS IN PERCENT OF CORRECT VALUE OBTAINED FROM EXPERIMENTAL EVALUATION

$\begin{array}{clr}\text { Wavelength }[\mu \mathrm{m}] & 0.6328 & 1.15 \\ \text { Error in Percent for Gold Mirror } & -0.78 & -0.24 \\ \text { Error in Percent for Aluminum Mirror } & -0.82 & -0.31\end{array}$

Analyzing the data of Table VIII it is first obvious that the errors are weII within $1 \%$, the accuracy claimed for the integrating sphere reflectometer. Secondly, assuming that as a rough estimate, the reflectance of $\mathrm{BaSO}_{4}$ is about 0.95 for both wavelengths, we find from Table IV that the predicted error was about -0.15. This result shows a surprisingly good agreement with the theoretical analysis. It proves that the theoretical analysis is quite capable of predicting the sign as well as the order of magnitude of the errors. As a third observation, we find that within the precision of the measurement the errors are constant for a given wavelength. This finding immediately offers an explanation as to the cause of the difference, though small, between the predicted and the measured errors. Bi-directional reflectance measurements, such as presented in Appendix A for sodium chloride or in reference [11] for $\mathrm{MgO}$, show that for a given angle of incidence the directional distribution of reflected flux is a function of wavelength. Therefore, the deviation of the distribution from that of a perfect diffuser is also a function of wavelength. For an angle of incidence close to normal, two deviations from a cosine distribution are found. First, more flux is reflected into the direction of incidence than predicted on the basis of a perfect diffuser. Secondly, less flux is reflected at large polar angles than predicted by the cosine distribution. On analyzing the influence of these deviations on the integrating sphere measurements, we found the following. In the integrating sphere the incident jeam struck the sphere wall at a polar angle of $12^{\circ}$. The flux which is incident directly on the field of view of the detector has to leave this spot at polar angles of about $80^{\circ}$. This means that the first contribution of flux on the detector's field of view is smaller than predicted on the basis of perfectly diffuse reflection 
because:

a. more flux is lost out the entrance hole

b. less flux is reflected at polar angles of about $80^{\circ}$ than predicted on the basis of a cosine distribution.

Both would tend to decresse $S_{2}$, the signal when the beam is first incident on the sphere wall, which would result in a larger negative error than predicted. On the other hand, this decrease in $\mathrm{S}_{2}$ would somewhat counterbalance the error involved in the measurement of reflectance for a diffuse sample. In other words, if nondiffuseness of the coating is the reason that the negative errors obtained experimentali: for specular samples are larger than predicted on a theoretical basis, then the errors for a diftuse sample should be smaller than predicted in Section 2.

As a conclusion, we can say that the instrument is indeed capable of measuring absolute reflectance with an accuracy of better than $1 \%$ and a precision of about $0.2 \%$.

\subsection{High Temperature Reflectance Measurements}

After the calibration of the integrating sphere had been completed, all openings were sealed by quartz windows and the vacuum unit was connected to the sphere. Then a thoria sample was placed in the sample holder and the sample holder inserted into the sphere. Because of the vast amount of gas trapped in the $\mathrm{BaSO}_{4}$ coating, it took several days of continuous pumping to reach a pressure in the range of $1 \times 10^{-6}$ torr. To speed up outgassing of the coating, heating tapes were wrapped around the sphere and its temperature increased to about $80^{\circ} \mathrm{C}$. Although the thoria sample could be heated in air, vacuum had to be used because of the tungsten susceptor in which the thoria sample was placed. After a pressure of less than $1 \times 10^{-5}$ torr was reached, the measurements were started. First the room temperature reflectance was measured. Then the output of the RF-generator was slowly increased until a reading with the optical pyrometer was obtained. Right after the heating started, the pressure inside the sphere increased rapidly. The sample had to be kept at the same temperature until the vacuum system had lowered the pressure again. Reflectance measurements were made at steps of about $50^{\circ} \mathrm{C}$ in apparent brightness temperature as measured by an optical pyrometer. Since the temperature measurements form a very important part of the experiment, it seems worth while to take a closer look at the temperature measurements and the associated problems.

5.2.1. Temperature Measurements; As mentioned above and also earlier in Section 3, a micro-optica1 pyrometer was used to measure the brightness temperature of the sample. To make the measurements more convenient, a flat mirror and the pyrometer were mounted on a sliding bar with the guides permanently attached to the table. When the temperature was to be measured, the mirror-pyrometer assembly was brought into position. The mirror blocked the laser beam and the pyrometer was then focused onto the sample. No further adjustment was needed once the mirror and pyrometer assembly was in the correct position, (see Figure 37). 


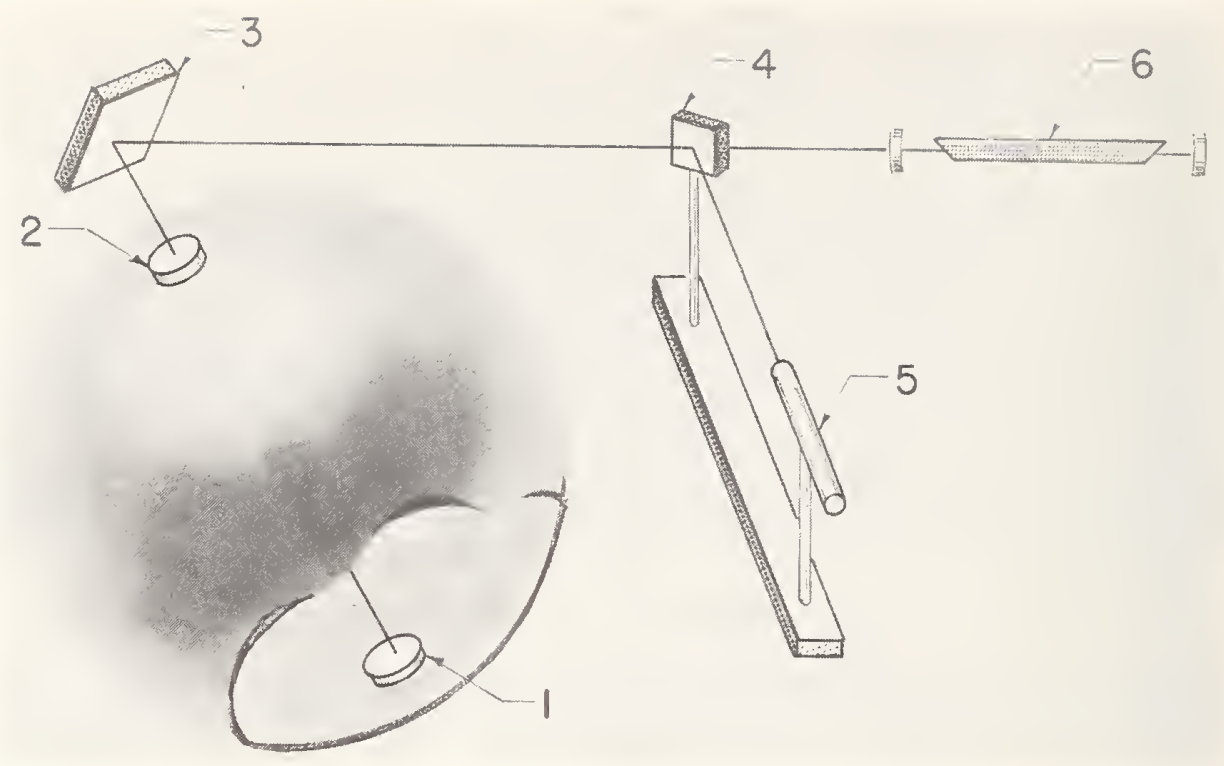

I. Sample

2. Quartz window

3. Flip-flop mirror

4. Folding mirror

5. Micro optical pyrometer

6. Laser

Figure 37. Temperature Measurement by a Micro-Optical Pyrometer

Since flux reaching the pyrometer was attenuated by the fused quartz window and the two folding mirrors, the brightness temperature indicated on the meter was not the brightness temperature of the sample. The measured brightness temperature is defined by the equation:

$$
L_{\lambda},(T)=\tau_{\text {eff, }, \lambda^{\prime}} \epsilon_{\lambda},(T) L_{b, \lambda},(T)=L_{b, \lambda},\left(T_{B}^{\prime}\right)
$$

where $\lambda^{\prime} \quad$ is the optical pyrometer mean effective wavelength $\left(\lambda^{\prime}=0.65 \mathrm{~m}\right)$

$L_{\lambda}$ ' the spectral radiance of the flux reaching the pyrometer at the wavelength $\lambda^{\prime}$.

$\mathrm{T}$ the true temperature of the sample in deg. Kelvin.

Teff the effective transmittance along the optical path from the sample to the pyrometer at the wavelength $\lambda^{\prime}$.

$\varepsilon_{\lambda},(T) \quad$ the spectral emittance of the sample at the wavelength $\lambda^{\prime}$ at the temperature of the sample. 
the spectral radiance of a blackbody at the wavelength $\lambda^{\prime}$ at the temperature of the sample.

and $\mathrm{T}_{\mathrm{B}}{ }^{\prime} \quad$ the apparent brightness temperature as measured by the optical pyrometer. The spectral radiance of a blackbody can be expressed by Planck's equation, namely

$$
L_{b, \lambda}(T)=\frac{e_{b, \lambda}(T)}{\pi}=\frac{1}{\pi} \frac{c_{1}}{n^{2} \lambda^{5}\left[\left(\exp c_{z} / n \lambda T\right)-1\right]},
$$

where $c_{1}$ and $c_{2}$ are constants

$\mathrm{n}$ is the index of refraction for the respective medium

$\mathrm{e}_{\mathrm{b}, \lambda}(\mathrm{T})$ is the spectral emissive power of a blackbody at the temperature $\mathrm{T}$. Substituting equation (34) into equation (33) and setting $n=1$ for air we obtain.

$$
\tau_{\text {eff }} \epsilon_{\lambda^{\prime}}(T)=\frac{\left(\exp c_{3} / \lambda^{\prime} T\right)-1}{\left(\exp c_{2} / \lambda^{\prime} T\right.}
$$

The numerical value for $c_{2}$ is $1.439 \mathrm{~cm}$ deg and the temperatures are in degrees Kelvin. Knowing $T_{\text {eff }}$ and $\epsilon_{,},(\mathrm{T})$ and having measured $\mathrm{T}_{B}^{\prime}$ one can obtain the true temperation $\mathrm{T}$ from equation (35).

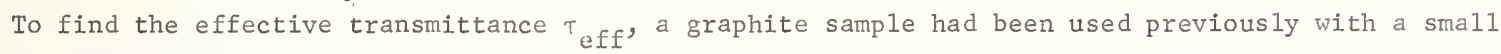
hole drilled from the side within about $0.5 \mathrm{~mm}$ of the front surface. A chromel-alumel thermocouple had then been adjusted such that the junction was in contact with the graphite. The sample was then slowly heated to about $1000^{\circ} \mathrm{K}$ and readings with the thermocouple and the optical pyrometer were taken. Knowing the true temperature and the brightness temperature, the product $\tau_{\text {eff }} \epsilon_{\lambda}$ (T) could be determined from equation (35). The function (35) has been tabulated by poland, et a1, [12] and these tables were used to find $T_{\text {eff }} \epsilon_{\lambda},(T)$. From simultaneous emittance measurements at $0.6328 \mu m$, $\tau_{\text {eff }}$ could then be established. The assumption was that the emittance at $0.6328 \mu \mathrm{m}$ was equal to the emittance at $0.65 \mu \mathrm{m}$. Since graphite is a very good approximation to a grey body, this was a very safe assumption to make. once $\tau$ eff has been found, the true temperature of a sample was determined simply by multiplying the spectral emittance, measured at this given temperature at $0.6328 \mu \mathrm{m}$, by $\tau$ eff and using the conversion tables.

5.2.2. Thoria: Having discussed the temperature measurements by an optical pyrometer, we can now return to the high temperature reflectance of thoria. Figure 38 shows the directional-hemispherical reflectance $\rho\left(12^{\circ}, 2 \pi\right)$ for $\mathrm{ThO}_{2}$ at $0.6328 \mu \mathrm{m}$ for three successive heating cycles as a function of the true sample temperature in ${ }^{\circ} \mathrm{K}$. The reflectance was first recorded as a function of apparent brightness temperature. This apparent brightness temperature was later corrected for the emittance of the sample and the attenuation of the radiation by the quartz windows and the two folding mirrors as discussed above. For the emittance correction, the emittance was computed from the reflectance measured for the apparent brightness temperature of the particular heating cycle. As can be seen from Figure 38 , the reflectance increased after each heating cycle. The room temperature reflectance increased from 0.829 before heating to 0.864 after the first heating cycle and to 0.873 after the second heating cycle. All three curves converge essentially at temperatures above $1800^{\circ} \mathrm{K}$.

Raising the temperature of the sample was always accompanied by an increase of pressure inside the sphere. The higher the temperature, the greater the rise in pressure became for a given temperature increment. The increase in pressure was probably due to two factors, outgassing of the sample itself and outgassing of the sphere coating whose temperature had been increased due to emitted flux from the sample and the tungsten susceptor to a value well beyond the temperature maintained during bakeout. The measurements were stopped when the pressure had increased to around $1 \times 10^{-3}$ torr. This then determined the highest sample temperature at which measurements were taken. 


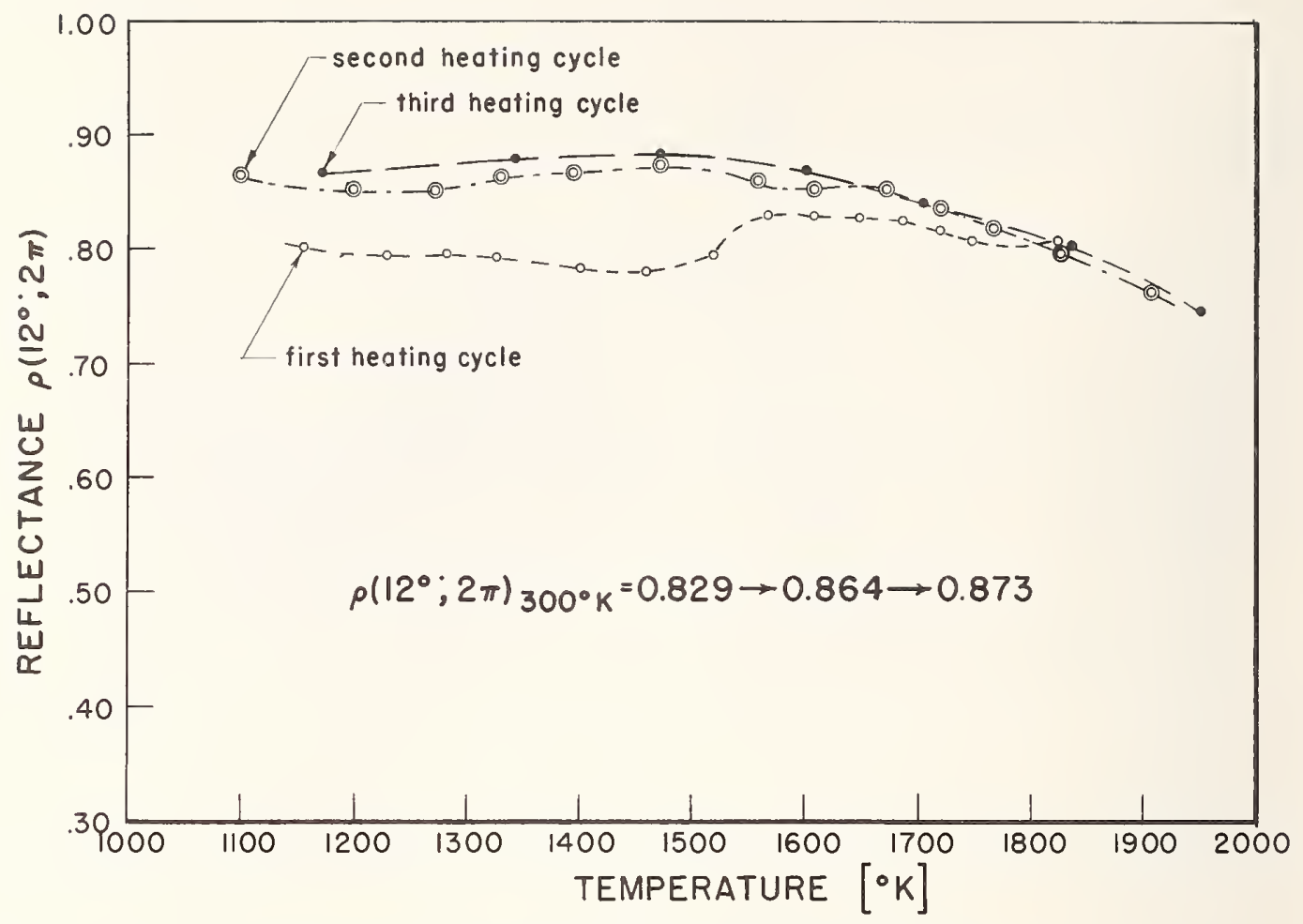

Figure 38. Directional-Hemispherical Reflectance $p\left(12^{\circ}: 2 \pi\right)$ of $\mathrm{ThO}_{2}$ at $0.6328 \mu \mathrm{m}$

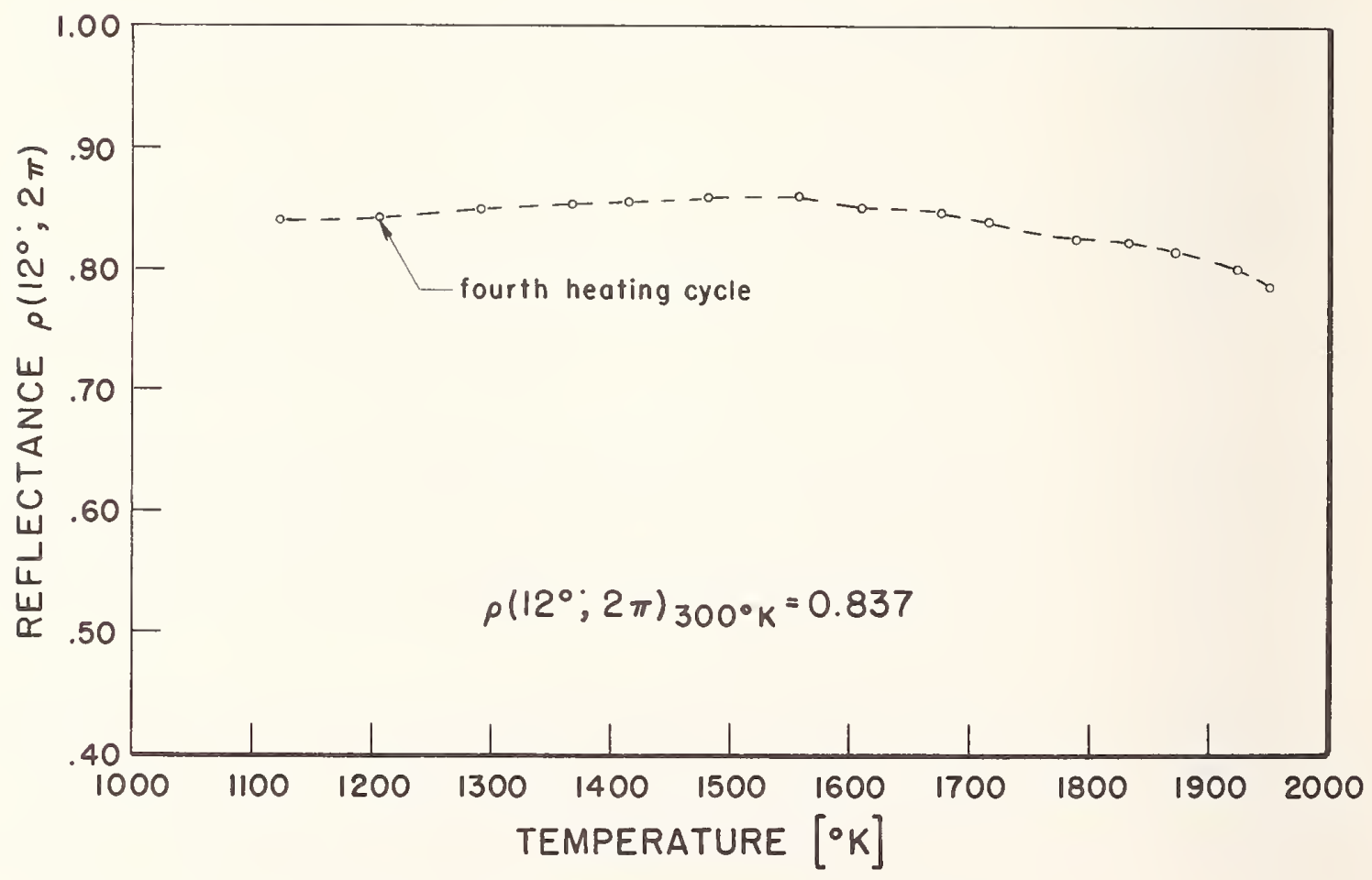

Figure 39. Directional-Hemispherical Reflectance $p\left(1,{ }^{\circ} ; 2 \pi\right)$ of $\mathrm{ThO}_{2}$ at $1.15 \mu \mathrm{m}$ 
Figure 39 gives the directional-hemispherical veflectance of the same samplo during the fourth heating cycle but this time measured at $1.15 \mu \mathrm{m}$. The reflectance values measured during the third heating cycle at $0.6328 \mu \mathrm{m}$ were used for the conversion of the brightness temperature to the true temperature. From Figure 39 ore could conclude that the thoria sample still had not reached an equilibrium condition. As mentioned in Section 4, the sample appeared brighter after the four heating cycles when compared with an unheated sample.

5.2.3. Graphite: The next sample measured was of graphite. The electrical conductivity of graphite is high enough so that it can act as its own susceptor. Therefore, the tungsten susceptor was replaced by an alumina crucible in to which the graphite sample was placed. The time required to reach about $1 \times 10^{-5}$ torr had now decreased to about three hours. Figure 40 shows $p\left(12^{\circ} ; 2 \pi\right)$ of graphite as a function of the sample temperature. At $0.6328 \mu \mathrm{m}$, the reflectance was almost independent of temperature. The room temperature reflectance increased from 0.258 before heating to 0.263 after the first heating cycle. The values taken during the second heating cycle are slightly higher over the whols temperature range. During the third and fourth cycle, the reflectance was measured at 1.15 m. At this wavelength, the reflectance decreases slightly with increasing temperature from 0.331 at room temperature to 0.305 at $2160^{\circ} \mathrm{K}$. The values taken during the fourth heating cycle are on the average somewhat higher than those measured during the third cycle. This is also indicated by a change in rom temperature reflectance from 0.330 before the third cycle to 0.331 after the third heating cycle. Table IX gives $p\left(12^{\circ} ; 2 \pi\right)$ obtained during each of the four heating cycles. As can be seen from the table, a higl precision can be obtained by this instrument. It should be mentioned, however, that the data on graphite were taken with a Perkin-Elmer Model 3300 laser which has a nominal output of $15 \mathrm{~mW}$ as compared with $1.5 \mathrm{~mW}$ of the Model 5220, which was used for the other matırials. The increase in signal-to-noj.se ratio by a factor of 10 is responsiole for the higher precision of the graphite data. It appears that the precision was at least 0.0005 . This can be seen from the data taken during the second, third and fourti! heating cycle. Although the decrease in reflect.nce over the whole temperature range was very small, tinere was a continuous decrease in the measured values. This is especially striking at $0.6328 \mu m$ where the total change from rom temperature up to $2141^{\circ} \mathrm{K}$ was only 0.0042 .

5.2.4. Tungsten: The list sample investigated was of tungsten. Firure 41 shon $p\left(12^{\circ} ; 2 \pi\right)$ for tungsten for three successive heating cycles. The reflectance was measured at $0.6328 \mu m$ during the first and secorld cycle and at $1,15 \mu \mathrm{m}$ during the third cycla. The pressure at the beginning of the heating was about $5 \times 10^{-6}$ and reached $5 \times 10^{-4}$ at the highest temperature. Duriig the first cycle, the reflectance changed quite irregularly. First the reflectance remained alnost constant then decreased slightly around $1400^{\circ} \mathrm{K}$ followed by a sharper increase around $1500^{\circ} \mathrm{K}$. Another sharp iiicrease occurred between $1760^{\circ} \mathrm{K}$ and $1960^{\circ} \mathrm{K}$. The rom temperature reflectance had increased from 0.502 before heating to 0.545 after the first cycle. During the second heating cycle, the reflectance increased slowly as it should according to other investigators [13], [14] and [15]. Around $1900^{\circ} \mathrm{K}$ another change occurred which caused the reflectance to drop. The room temperature reflectance had decreased from 0.545 at the beginning of the second cycle to 0.528 after the second cycle. At $1.15 \mu \mathrm{m}$, the reflectance decreased slowly in a 1 near fashion. If this decrease is truly an effect of temperature, then this would indisate tiat the crossover point, defined as the wavelength for which $\partial \varepsilon / \partial \mathrm{I}^{2}=0$, would be at a wavelength shorter than $1.15 \mu \mathrm{m}$. This finding would not agree with the measurements by DeVos [13] who found that the crossorer point for tungsten occurs at $1.27 \mu \mathrm{m}$. As we have seen from Figures 26 up to and inclinding 34 , the surface had changed considerably so that the decrease in reflectance could also be due to deterioration of the surface of the sample. Table X compares the data obtained by DeVos and Ornstein [14] with tine data taken with the integrating sphere. The data by DeVos and Ornstein were taken from page 46 and 48 of Svet [16]. The data given by Svet are spectral, normal emittance data; and, therefore, the data presented as integrating sphere data are actially calculated from the reflactance data. As can be seen from Table $\mathrm{X}$, the data points for the two heating cycles agree within $0.5 \%$ of reflectance except for the 


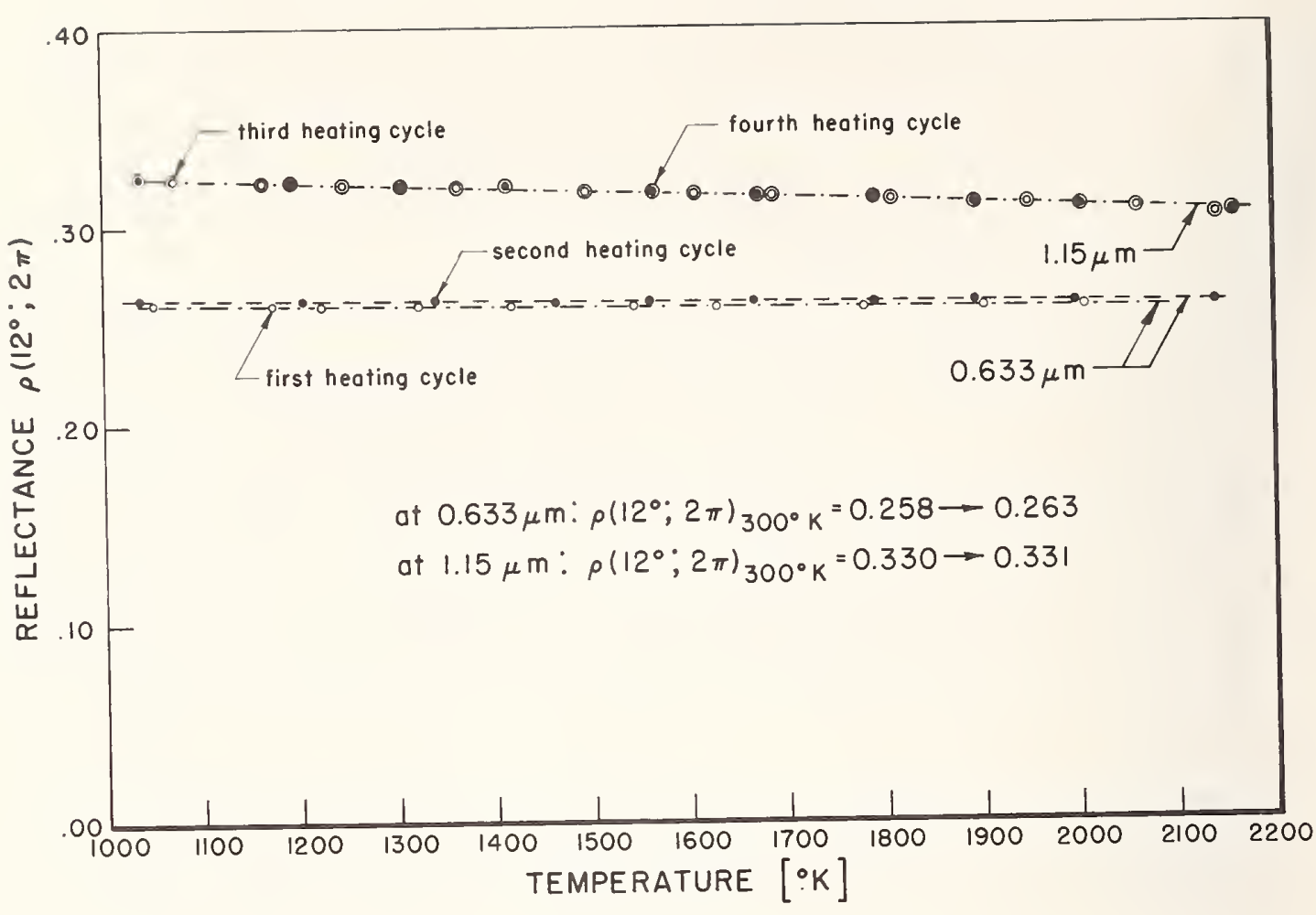

Figure 40. Directiona1-Hemispherical Reflectance $p\left(12^{\circ} ; 2 \pi\right)$ of Graphite at 0.6328 and $1.15 \mu \mathrm{m}$

TABLE IX

REFLECTANCE OF GRAPHITE AT 0.6328 and $1.15 \mathrm{um}$ FOR FOUR SUCCESSIVE HEATING CYCLES

\begin{tabular}{lrrrrrrr}
\hline \multicolumn{2}{c}{$0.6328 \mu \mathrm{m}$} \\
\hline \multicolumn{2}{c}{ first cycle } & \multicolumn{2}{c}{ second cycle } & \multicolumn{2}{c}{ third cycle } & \multicolumn{2}{c}{ fourth cycle } \\
\hline$\rho\left(12^{\circ} ; 2 \pi\right)$ & $\mathrm{T}\left[{ }^{\circ} \mathrm{K}\right]$ & $\rho\left(12^{\circ} ; \pi\right)$ & $\mathrm{T}\left[{ }^{\circ} \mathrm{K}\right]$ & $\rho\left(12^{\circ}: 2 \pi\right)$ & $\mathrm{T}\left[{ }^{\circ} \mathrm{K}\right]$ & $\rho\left(12^{\circ}: 2 \pi\right)$ & $\mathrm{T}\left[{ }^{\circ} \mathrm{K}\right]$ \\
\hline 0.2577 & 300 & 0.2629 & 300 & 0.3301 & 300 & 0.3310 & 300 \\
0.2593 & 1052 & 0.2625 & 1038 & 0.3234 & 1076 & 0.3249 & 1039 \\
0.2589 & 1175 & 0.2622 & 1205 & 0.3217 & 1167 & 0.3227 & 1195 \\
0.2590 & 1226 & 0.2614 & 1343 & 0.3206 & 1249 & 0.3210 & 1309 \\
0.2595 & 1326 & 0.2611 & 1464 & 0.3191 & 1366 & 0.3200 & 1417 \\
0.2584 & 1420 & 0.2608 & 1516 & 0.3172 & 1497 & 0.3169 & 1566 \\
0.2584 & 1544 & 0.2603 & 1668 & 0.3156 & 1609 & 0.3152 & 1673 \\
0.2581 & 1629 & 0.2601 & 1792 & 0.3137 & 1689 & 0.3131 & 1792 \\
0.2575 & 1782 & 0.2596 & 1894 & 0.3114 & 1809 & 0.3109 & 1897 \\
0.2578 & 1902 & 0.2592 & 1997 & 0.3088 & 1949 & 0.3086 & 2003 \\
0.2583 & 2005 & 0.2587 & 2141 & 0.3076 & 2062 & 0.3054 & 2161 \\
& & & & 0.3045 & 2144 & & \\
\hline
\end{tabular}


point at $2000^{\circ} \mathrm{K}$. As it was noticed earlier, a drop in reflectance occurred around $1875^{\circ} \mathrm{K}$. If we extrapolate the data beyond $1850^{\circ} \mathrm{K}$, which is simple because the increase in reflectance is very nearly a linear function of the temperature, then the emittance at $2000^{\circ} \mathrm{K}$ would be 0.433 which would agree with DeVos' data point. All emittance data at $1.15 \mu \mathrm{m}$ taken with the sphere are higher than those taken by Onnstein. This again could be due to surface deterioration of the sample. Unfortunately, neither DeVos nor Ornstein discussed the surface conditions of their samples.

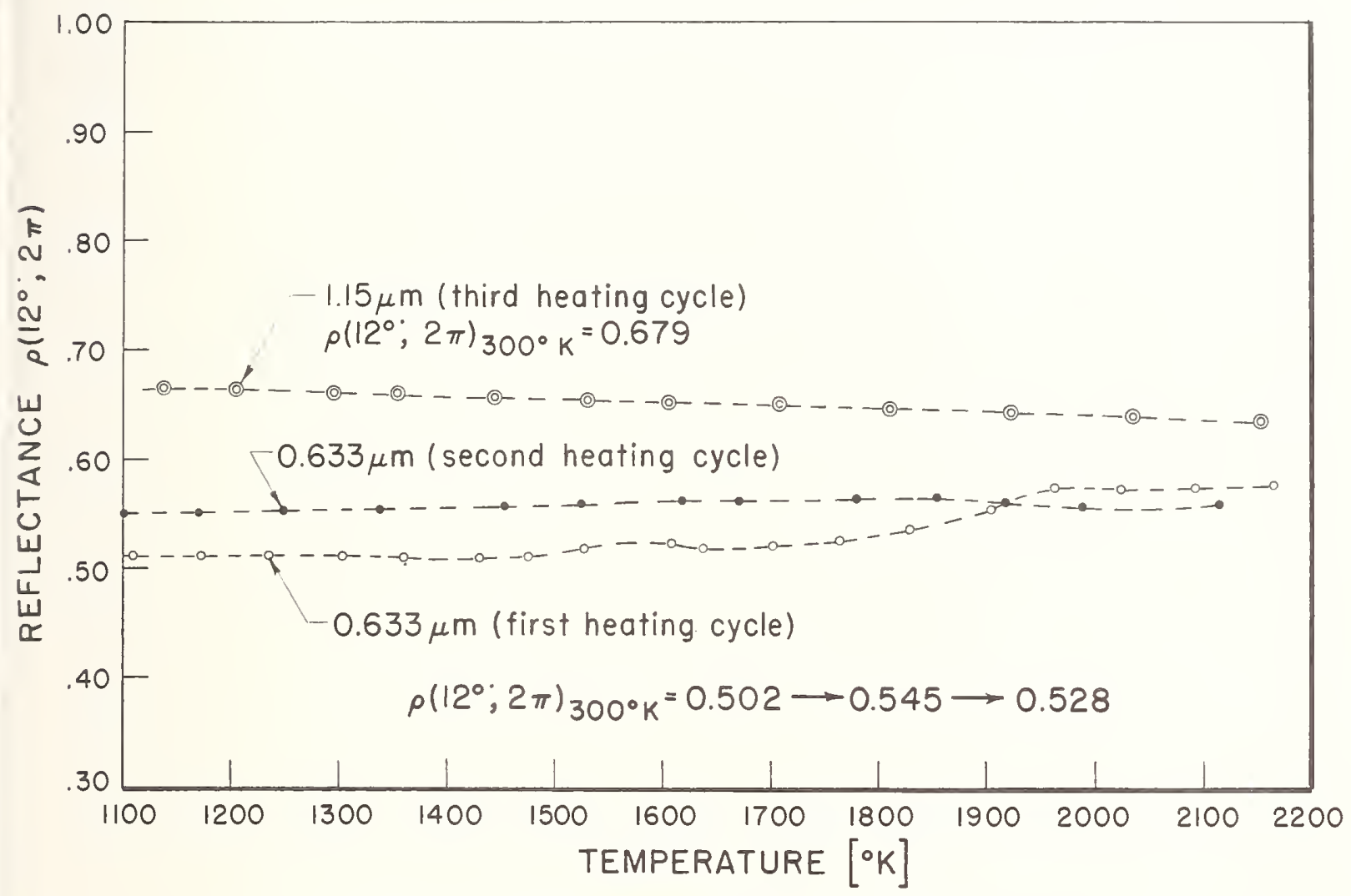

Figure 41. Directiona1-Hemispherical Reflectance $p\left(12^{\circ} ; 2 \pi\right)$ of Tungsten at 0.5328 and $1.15 \mu$ m

TABLE X

NORMAL EMTTTANCE OF

TUNGSTEN AT 0.6328 AND $1.15 \mu \mathrm{m}$

\begin{tabular}{|c|c|c|c|c|c|c|c|}
\hline & True Temperature $\left[{ }^{\circ} \mathrm{K}\right]$ & & 1200 & 1400 & 1600 & 1800 & 2000 \\
\hline \multirow{3}{*}{$0.6328 \mu \mathrm{m}$} & Integrating Sphere & Cycle 1 & 0.488 & 0.491 & 0.476 & 0.437 & 0.426 \\
\hline & & Cycle 2 & 0.448 & 0.445 & 0.440 & 0.437 & 0.445 \\
\hline & DeVos & & & & 0.442 & 0.438 & 0.434 \\
\hline \multirow[t]{2}{*}{$1.15 \mu \mathrm{m}$} & Integrating Sphere & Cycle 3 & 0.337 & 0.343 & 0.343 & 0.349 & 0.361 \\
\hline & Ornstein & & & & 0.331 & 0.321 & 0.310 \\
\hline
\end{tabular}




\section{CONCLUSIONS}

A laser source integrating sphere has been constructed which is capable of measuring the spectral, directional-hemispherical reflectance at very high temperatures for opaque materials. A maximum error of $1 \%$ had been predicted on the basis of a theoretical analysis. This accuracy is maintained regardless of the material, its surface structure and sample temperature. The results of the theoretical analysis were confirmed experimentally by using calibrated mirrors as reflectance standards. Finally the high temperature capability of the reflectometer was demonstrated by reflectance measurements on samples of thoria, graphite and tungsten at temperatures up to $2150^{\circ} \mathrm{K}$. The measurements on graphite also showed that the precision of the measurements is better than 0.0005 if a laser with a higher power output is used. The measured values obtained with this instrument can be used to compute spectral, directional emittance data with an accuracy not approached by any direct method. 
[1] McNicholas, H. J. "Absolute Methods in Reflectometry." I. Res. Natl. Bur. Std., Vol. 1, 1928, PP. $29-73$.

[2] Taylor, A. H. "Measurement of Diffuse Reflection Factors, and a New Absolute Reflectometer." Scientific Papers of the Bureau of Standards, Vol. 16, 1920, pp. 421-436.

[3] Hamilton, D. C. and Morgan, W. R. "Radiant-Interchange Configuration Factors." NACA TN $2836,1952$.

[4] Dunn, S. T., Richmond, J. C., and Wiebelt, J. A. "Ellipsoidal Mirror Reflectometer." J. Res. Nat1. Bur. Std., Vol. 70C, No. 2, 1966, Pp. 75-88.

[5] Bloom, Arnold L. "Properties of Laser Resonators Giving Uniphase Wave Fronts." Spectra-Physics Laser Technical Bulletin, No. 2, Published by Spectra Physics, Inc., Mountain View, Calif.

[6] Mak, F. and Sellers, C. M. "A Simple Method of Inert Gas Purification." I. Sci. Instr., Vol. 42, 1965, PP. $900-901$.

[7] Bennett, H. E. and Bennett, Jean M. "Precision Measurements in Thin Film Optics." Physics of Thin Films, Vol. 4, ed. G. Hass and R. Thun. To be published by Academic Press in Nov. 1967.

[8] Wiebélt, J. A. Engineering Radiation Heat Transfer. Holt, Rinehart \& Winston Publishing Company, 1966.

[9] Sparrow, E. M. and Cess, R. D. Radiation Heat Transfer. Brooks/Cole Publishing Company, Belmont, Calif., 1966.

[10] Hass, G. Optics Section American Institute of Physics Handbook, ed. Gray, D. E., 1957, McGraw-Hil1 Book Company, Inc., New York.

[11] Miller, E. R. and Kannon, R. S. "Development and Use of a Bidirectional Spectroreflectometer." AIAA Paper No. 67-313, presented at AIAA Thermophysics Specialist Conference, New Orleans, April 1967.

[12] Poland, D. E., Green, J. W., and Mangrave, J. L. Corrected Optical Pyrometer Readings. NBS Morograph 30, 1961 .

[13] DeVos, J. C. "A New Determination of the Emissivity of Tungsten Ribbon." Physica, No. 20, 1954, PP. 690-714.

[14] Ornstein, L. S. "Tables of the Emissivity of Tungsten as a Function of Wavelength from $0.23-2.0 \mu$ in tise Region of Temperature of 1600-3000\%." Physica, No. 3, 1936, p. 561.

[15] Larrabee, R. D. "Spectral Emissivity of Tungsten." J. Opt. Soc. Am., Vo1. 49, No. 6, 1959, p. 619.

[16] Svet, Darii Yakovlevich. Thermal Radiation. Authorized translation from the Russian, Consultants Bureau, New York, 1965.

[17] Ballard, S. Stanley, McCarthy, A. Kathryn, and Wolfe, I. William. "Optical Materials for Infrared Instrumentation." IRIA Report 2389-11-S, 1959.

[13] Edwards, D. K., Gier, J. T., Nelson, K. E. and Roddick, R. D. "Integrating Sphere for Imperfectly Di:fuse Samples." Applied Optics, Vol 51, 1961 pp. 1279-1288.

[19] White, John W. "New Method Eor Measuring Diffuse Reflectance in the Infrared." J. Opt. Soc. Am., Vo1. 54, 1964, Pp. 1322-1337.

[20] Judd, D. B. "Terms, Definitions, and Symbols in Reflectometry." I. Opt. Soc. Am., Vol. 57, 1967, Pp. 445-452.

[21] Sommerfeld, Arnold. "Das Reziprozitats-Theorem der drahtlosen Telegraphie." Jahrbuch der drahtlosen Telegraphie, Vol. 26, 1925, pp. 93-98.

[22] Fragstein, C. vor. "Über die Formulierung des Kirchhoffschen Gesetzes und ihre Bedeutung fur eine zwecknassige Definition von Remissionszahlen." Octik, Vol, 12, 1955, pp. 60-69.

[23] Nicodemus, Fred E. "Directional Reflectance and Emissivity of an Opaque Surface." Applied Optics, Vol. 4, 1965, pp. 767-773. 
8. Appendix A

THE USE OF SODIUM CHLORIDE AS AN

IINTEGRATING SPHERE COATING

A coating which was to be used for this integrating sphere had to meet several requirements. First of a11, the coating had to be a very good diffuser of uniform reflectance since the theory of the integrating sphere is based on the assumption that the coating is uniform and a perfectly diffuse reflector. Then, as can be seen from equations (14), (19) and (21) derived in Section 2, the flux incident on the detector's field of view is a very strong function of the wall reflectance. Therefore, it is advantageous to have a highly reflective coating, even more so, since the systematic error for a diffuse sample decreases linearly with increasing wall reflectance. (See Figure 10.) While these first two requirements are common for any type of integrating sphere, there are several properties which a coating for this particular sphere had to possess. Part of the coating was very close to the heated sample; and therefore, reached a high temperature. This means that the coating had to withstand high temperatures without undergoing physical or chemical changes which could alter the diffusing property or the reflectance of the coating. Since it was desirable to reach a vacuum of $10^{-5}$ or $10^{-6}$ torr, the coating should not increase the gas load on a vacuum system to a point where this pressure could not be maintained.

None of the sphere coatings commonly used, such as magnesium oxide, barium sulfate and sulfur, meet al1 of these requirements. The orily coating used beyond $2.5 \mu \mathrm{m}$ is sulfur. The mechanism by which sulfur becomes a good diffuser is body scattering. The incident flux is partly reflected on the surface. The greater part penetrates into the coating and is scattered by randomly oriented crystallites, and if the layer is thick enough, most of the flux is backscattered in a diffuse manner. Therefore, the substance to look for was a material with as low an absorption coefficient as possible within the wavelength regio of interest applied as a polycrystalline coating. The material finally chosen was sodium chloride. Fron

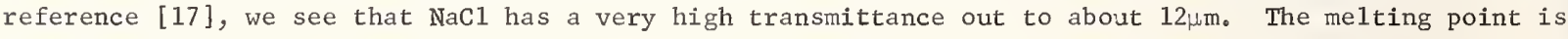
given as $801^{\prime 2} \mathrm{C}$.

After it had been decided to use NaC1 as a sphere coating, a method had to be found to apply the salt to the surface. First a saturated solution of $\mathrm{NaCl}$ in water was heated to about $80^{\circ} \mathrm{C}$ and sprayed orto the substrate. This method was abandoned because of long drying times between successive coatings, difficulties in controlling the size of the crystals formed and excessive corrosion of the spray equipment. Since good results had been obtained with barium sulfate suspended in alcohol, the same technique was tried with NaCl. Reagent grade NaCl and ethyl alcohol were ground in a bal1 nill for approximately 48 hours. No effort was made to actually measure the average grain size. The suspension was then applied with a spray gun. Successive thin coatings were sprayed and then dried in an oven of about $70^{\circ} \mathrm{C}$ until the desired thickness was attained. Since measurements of directional-hemispherical reflectance had shown that the reflectance decreased considerably when the coating became rough, the layer of sodium chloride was smoothed several times during the process until a layer of about 1 tm was obtained. The finished coating was again smoothed by means of a $75 \mathrm{~mm}$ diameter glass disc which was ground to the curvature of the sphere. Flat samples were prepared to measure the directional-hemispherical reflectance in the wavelength range from 0.3 to $20 \mu \mathrm{m}$. The measurements in the range between 0.3 and $2.5 \mu m$ were made with an Edwards type integrating sphere [18] at Oklahoma State University. In the range from 2.5 to $19 \mu n$, the reflectance was measured with a Cary-White 90 infrared spectrophotometer [19] at the National Bureau of Standards. Although a study had been made to determine what thickness of Nacl was required to produce an essentially opaque layer, only the reflectance of the spzayed and smoothed salt coating is shown in Figure $\mathrm{A} 1$ and $\mathrm{A} 2$. These figures show that the reflectance is quite high throughout the visible and near infrared, with exception of two absorption bands around 2.9 and $3.4 \mu \mathrm{m}$. The reflectance is greater than $90 \%$ from 0.4 to $5.5 \mu \mathrm{m}$ with exception of the bands mentioned above, and drops below $80 \%$ beyond $7.8 \mu \mathrm{m}$. 
To establish the diffuseness of the flux reflected from the sodium chloride coating, a simple laser gonioreflectometer was built. The gonioreflectometer consisted of two turntables as used on milling machines, with angular scales that could be read to an accuracy of $0.05^{\circ}$. The two turntables were mounted above each other with a common axis of rotation. The upper table $\mathrm{T}_{2}$ carried an arm which was used to mount the detector, spikefilter, and light baffle. The source used was the same laser employed in the integrating sphere. Figure A3 shows a sketch of the gonioreflectometer and Figure A4 is a schematic of the total setup. To align the reflectometer, the sample was replaced by a flat, first surface mirror. The direction of $0^{\circ}$ incidence was established by adjusting the plane mirror $\mathrm{M}_{2}$ and the upper turntable $\mathrm{T}_{2}$ until the reflected beam coincided with the incident beam. This could be done by centering tile reflected beam around the diaphragm D (Figure A4). Then the 1ight baffle was mounted or the arm in a position such that the incident and reflected beams were centered in the diaphragms of the baffle. Finally, the iidex marks of the two turntables were set to read zero in this position, and the respective spikefilter and detector were mounted behind the baffle. The detector used was a $10 \mathrm{~mm} x 10 \mathrm{~mm}$ lead sulfide ce11. A spikefilter for the respective wavelength used was mounted in front of the detector and eliminated all spurious flux outside the transmission band. The light baffle, which consisted of a t tbe and a series of diaphragms, was painted with 3M-Velvet Black paint and restricted the detector's field of view to a somewhat larger area than the irradiated area of the sample. Even at large angles of incilence (up to $75^{\circ}$ ), the detector always viewed the entire irradiated area. Under these viewing conditions, the signal measured by the detector if the sample were a perfect diffuser would be described by the equation

$$
S=S_{\max } \cos \theta^{\prime}
$$

where $\theta^{\prime}$ is the angle between the normal of the surface and the direction of viewing, S is the signal at a given angle $\theta^{\prime}$ and $S_{\max }$ is the maximum signal ottained when the detector views the sample in the normal direction. (See Figure A5).

The angles which coula be adjusted by means of the two turntables were $Y$ and $\delta$ (see Figure A4), where $\gamma$ is the angle between the direction of incidence and the direction of viawing and $\delta$ is the angle between the normal to the surface and the direction of viewing. Therefore, the desired angle of incidence $\theta$ and angle of exitance $\theta^{\prime}$ can be ottained from the simple relationships

$$
\theta^{\prime}=.8
$$

and

$$
\theta=\gamma-\delta \text {. }
$$

To check tine accuracy of the angle settings, the distribution of the reflected flux from a first surface mirror was measured. The angle of incidence was set at $10.00^{\circ}$ and then data were taken between $\theta^{\prime}=9^{\circ}$ and $\theta^{\prime}=12^{\circ}$. Around $\theta^{\prime}=10^{\circ}$ measurements were made at $0.05^{\circ}$ intervals. These data are given in Figure $A 6$ which shows that the maximum is at $\theta^{\prime}=10.03^{\circ}$ instead of $\theta^{\prime}=10.00^{\circ}$. This error 1 ies within the accuracy to which the turntables could be set. 


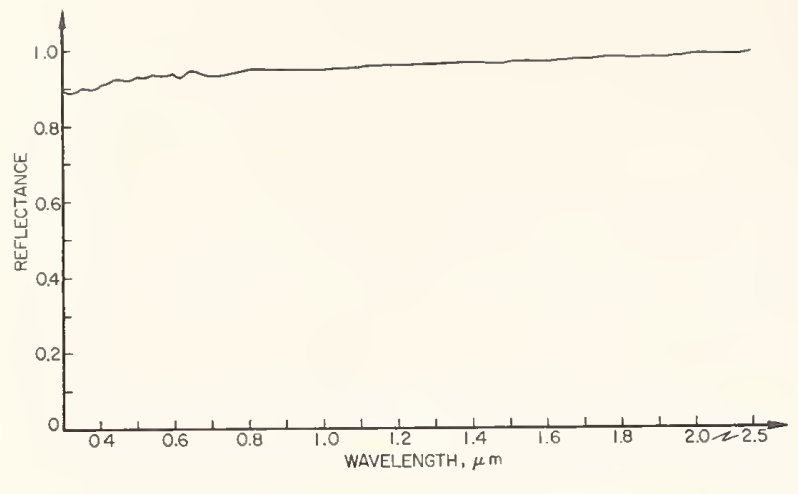

Figure A1. Reflectance of Sodium Chloride

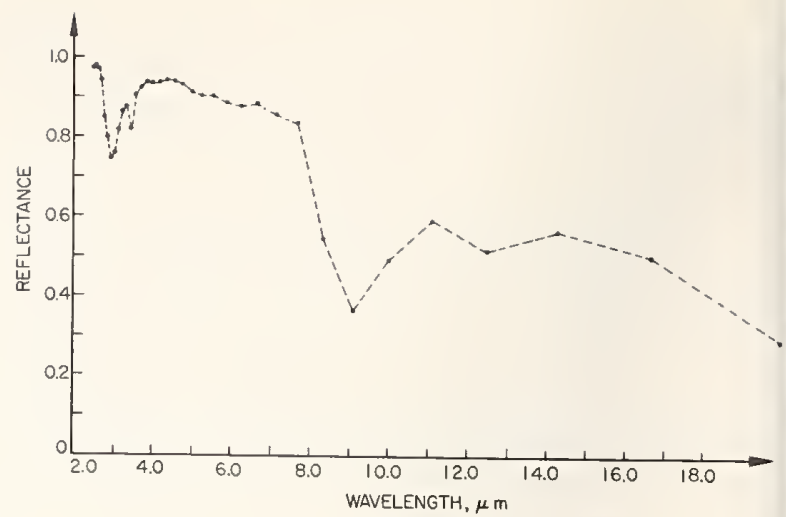

Figure A2. Reflectance of Sodium Chloride

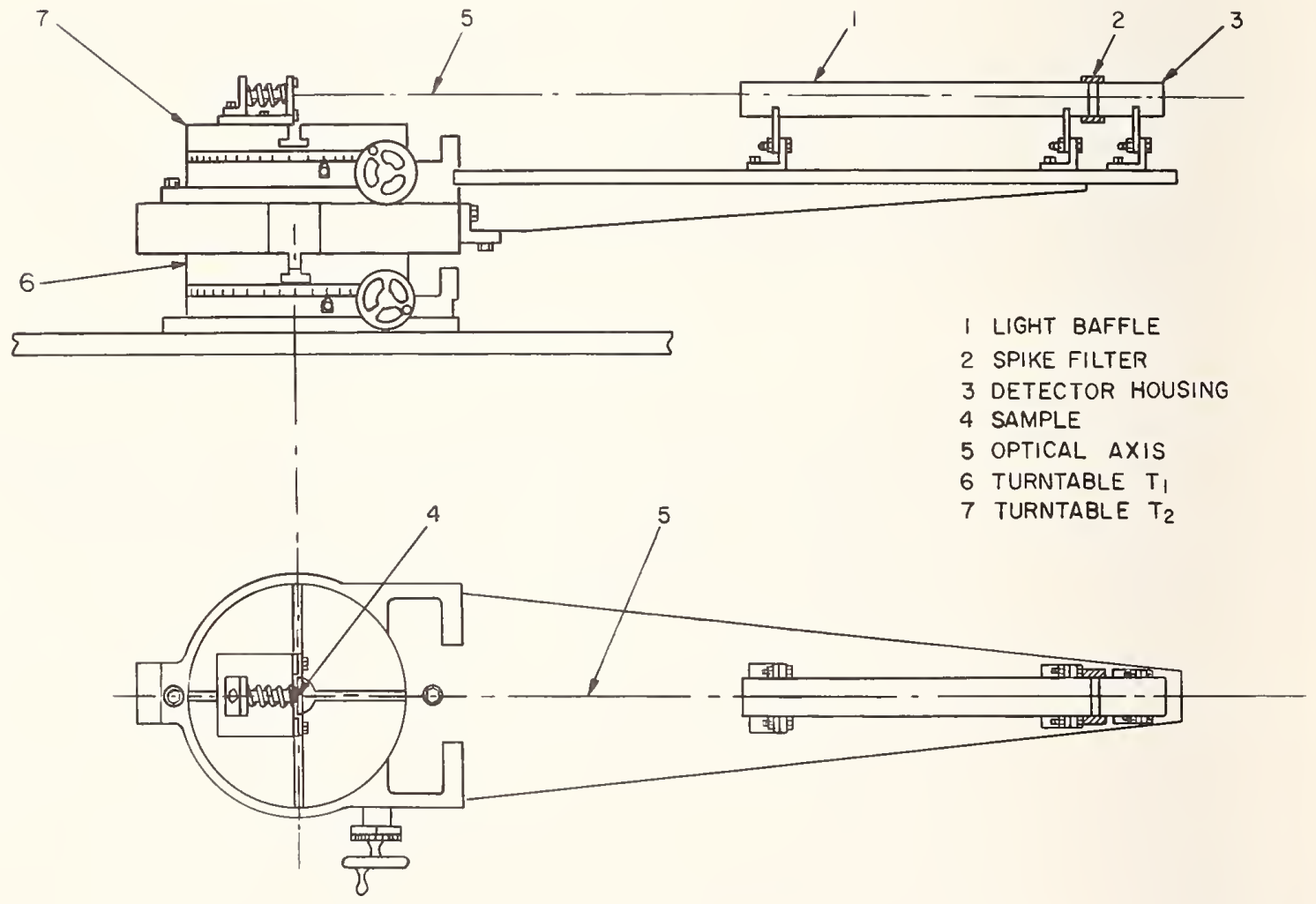

Figure A3. Gonioreflectometer 

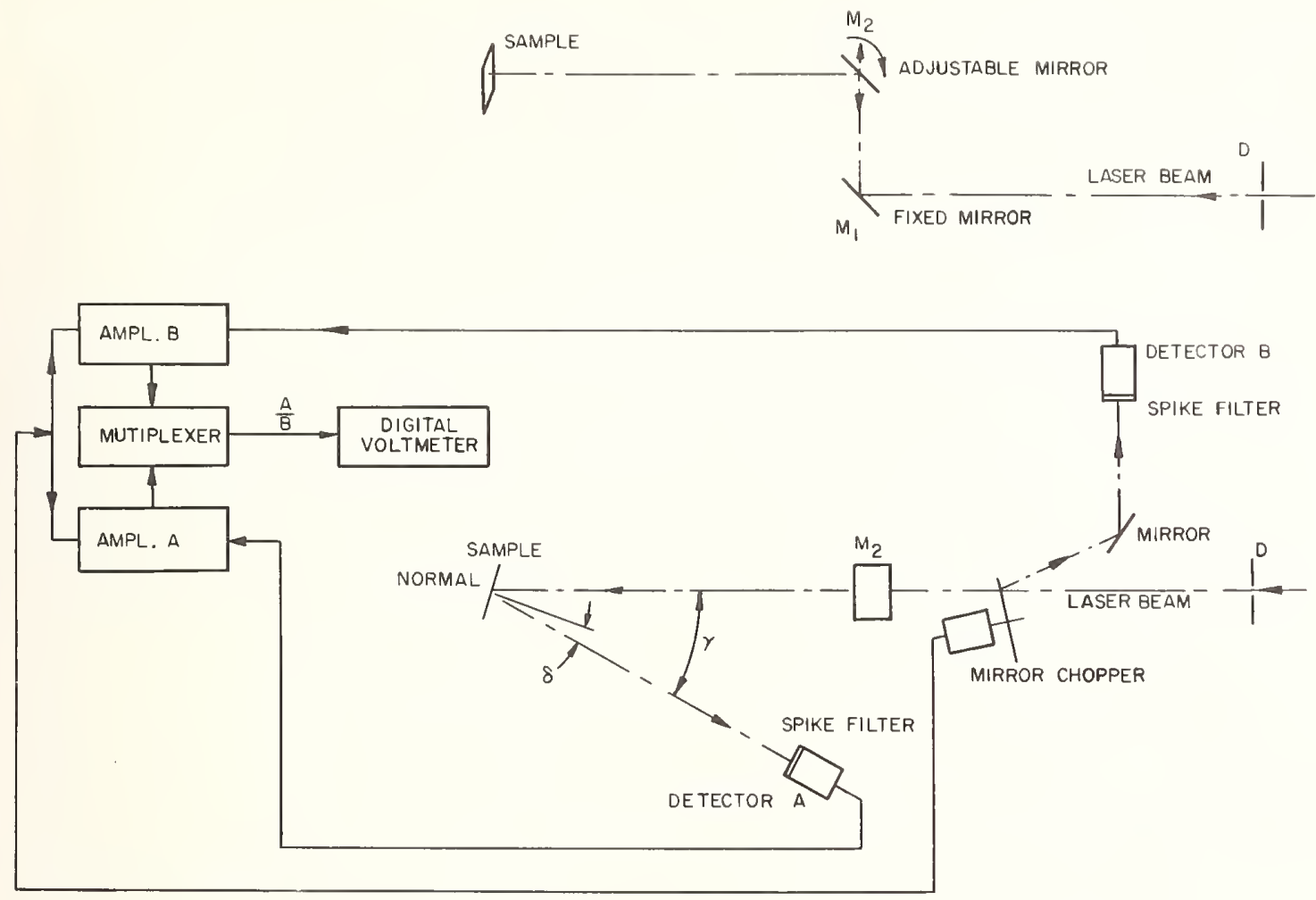

Figure A4. Schematic and Signal Flow Diagram of Gonioreflectometer

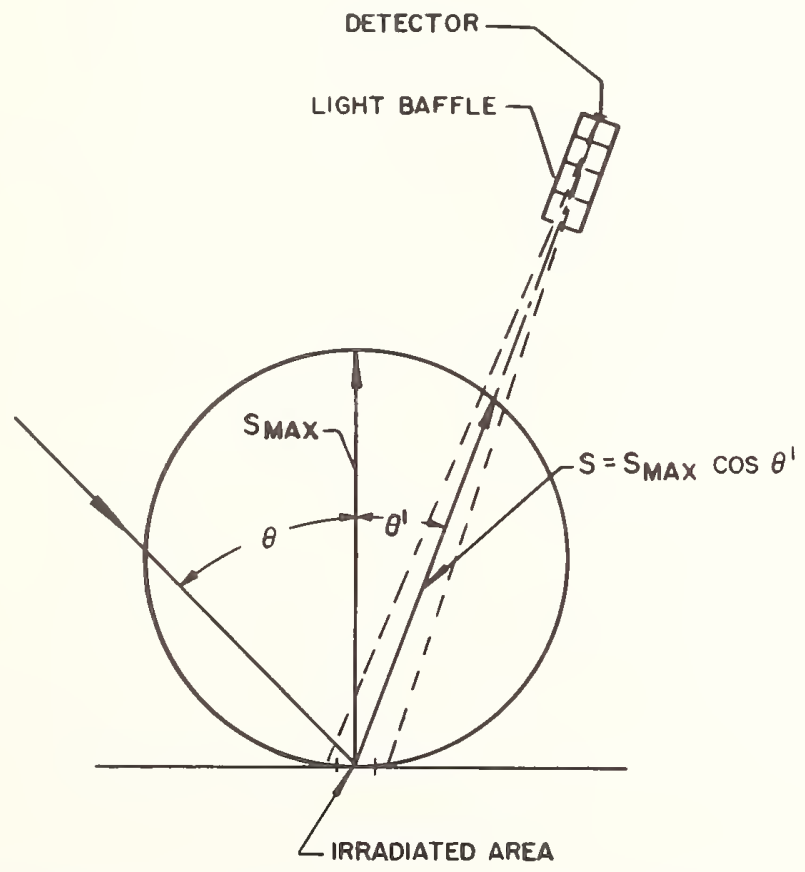

Figure A5. Flux Distribution of a Perfect Dizfuser 


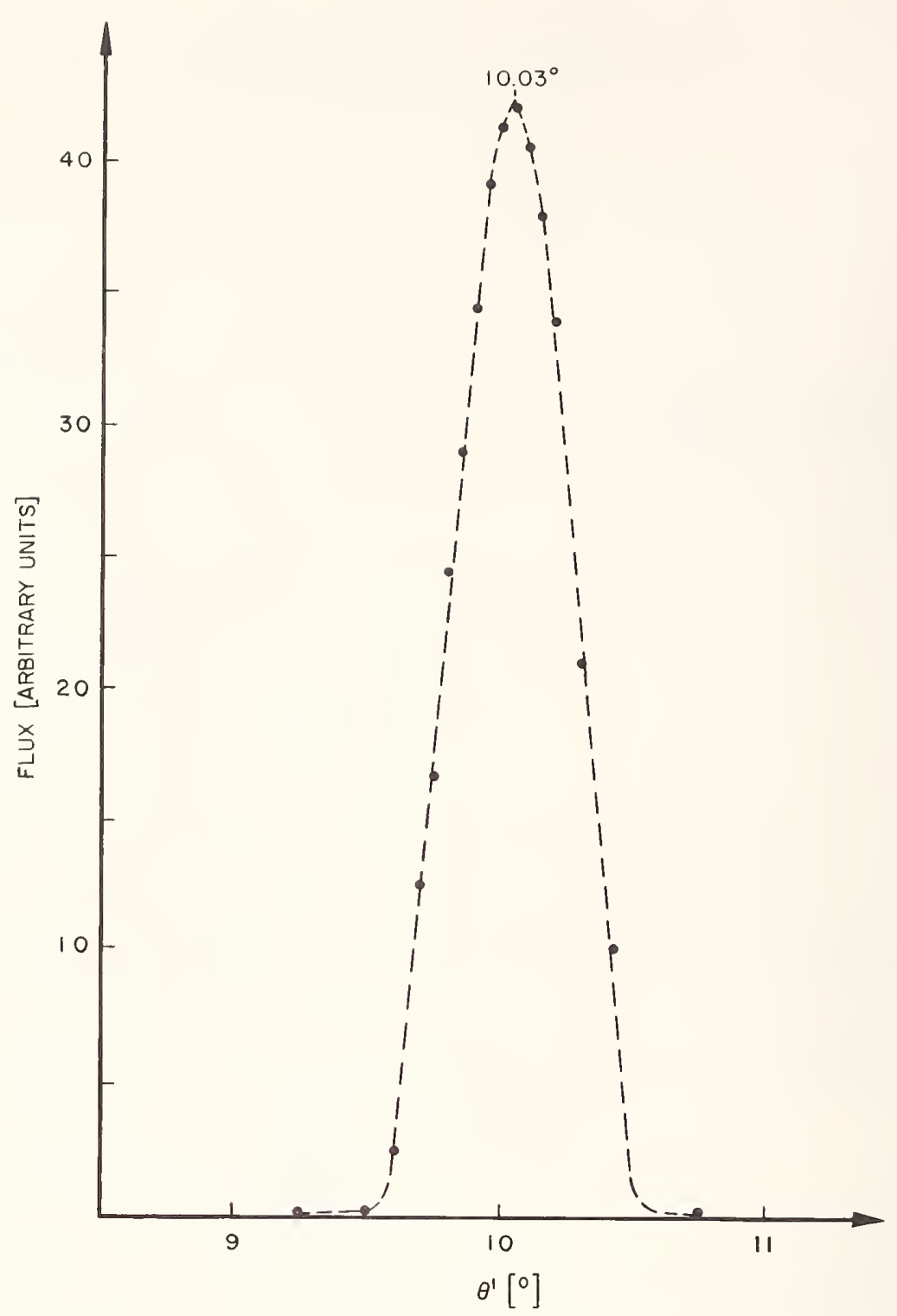

Figure A6. Directiona1 Distribution of Reflected Flux for a Mirror

After the gonioreflectometer was aligned, the mirror was replaced by the sample to be measured. For the measurements at 0.6328 and $1.15 \mu \mathrm{m}$, the distance from the detector to the center of the irradiated area was approximately $105 \mathrm{~cm}$, which, together with a detector area of $1 \mathrm{~cm}^{2}$, corresponds to an acceptance solid angle of $0.91 \times 10^{-4}$ ster. At 0.6328 and $1.15 \mu n$, measurements were taken at $5^{\circ}$ intervals starting at $\theta^{\prime}=85$. Since there were indications of a slight peak in the retrodirection, measurements were made as close to the direction of incidence as possible, which was $3^{\circ}$ on either side. Also readings were taken every $2.5^{\circ}$ around the specular direction to detect any specular peak if present. At any given angle setting, 10 consecutive readings from the digital voltmeter were averaged. This was also done for the background, which was then subtracted from the signal. 
To take one scan through the plane of incidence required approximately $11 / 2$ hours. Since one cannot rely on the output of the laser to remain constant during that period of time, the same ratioing technique as that used with the integrating sphere reflectometer was applied (see Figure A4). The maximum signal difference experienced in comparing two measurements for the same angle setting at the beginning and the end of a scan was $0.4 \%$.

Figures $A 7, A 8, A 9$ and $A 10$ show the distribution of the reflected flux from the NaC1 coating at $0.6328 \mu \mathrm{m}$ at angles of incidence of $0,25,50$ and $75^{\circ}$. The units are arbitrary. After all data for one wavelength were taken, the flux reflected at $\theta^{\prime}=10^{\circ}$ was measured for all four angles of incidence. Then the signals for $\theta^{\prime}=10^{\circ}$ of the four sets previously measured were scaled to yield the same ratios as the signals just obtained. The signals for all other $\theta^{\prime}$ were adjusted accordingly. This makes it possible to compare the fluxes reflected into a particular direction $\theta^{\prime}$ for any of the angles of incidence used. As can be seen from the figures, the flux reflected at angles close to the normal decreases with increasing angle of incidence. At large angles of incidence, more flux is reflected at large polar angles. There was hardly any indication of a specular peak, while a definite peak in the retrodirection could be found. Figures A11, A12, A13 and A14 show the same sets of data for 1.15 m.

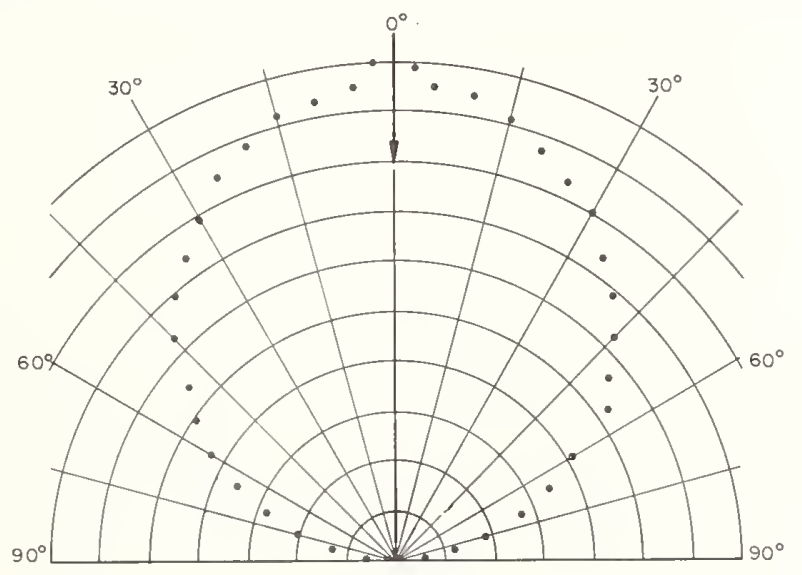

Figure A7. Directional Distribution of Reflected Flux for Sodium Chloride at $0.6328 \mu \mathrm{n}$ and $\theta=0^{\circ}$

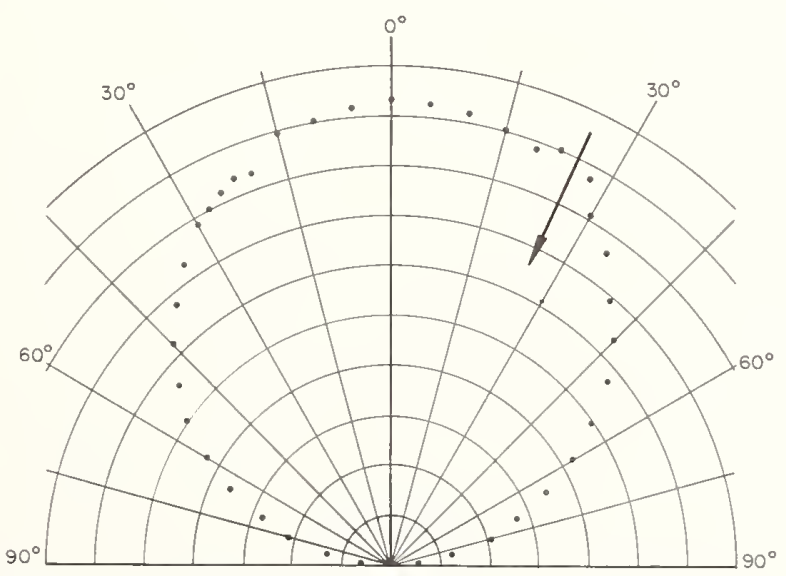

Figure A8. Directiona1 Distribution of Reflected Flux for Sodium Chloride at $0.6328 \mu m$ and $\theta=25^{\circ}$ 


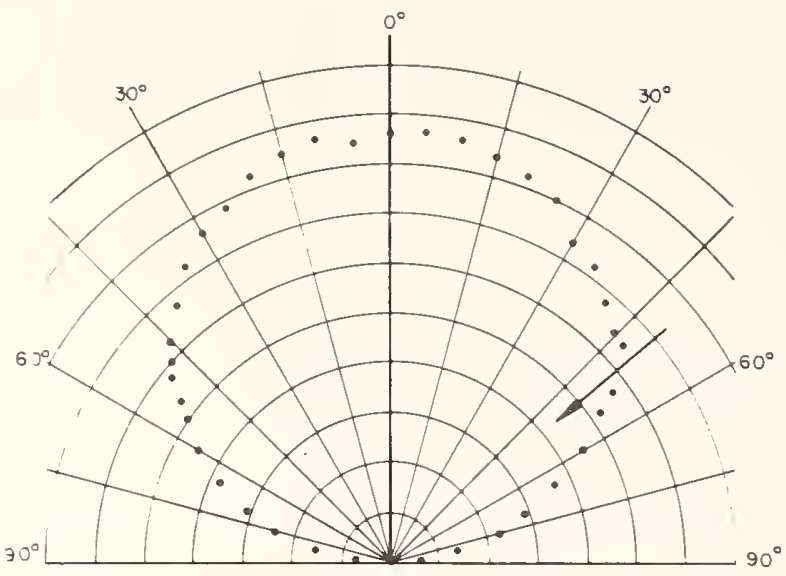

Figure A9. Directional Distribution of Reflected Flux for Sodium Chloride at $0.6328 \mu \mathrm{n}$ and $\theta=50^{\circ}$

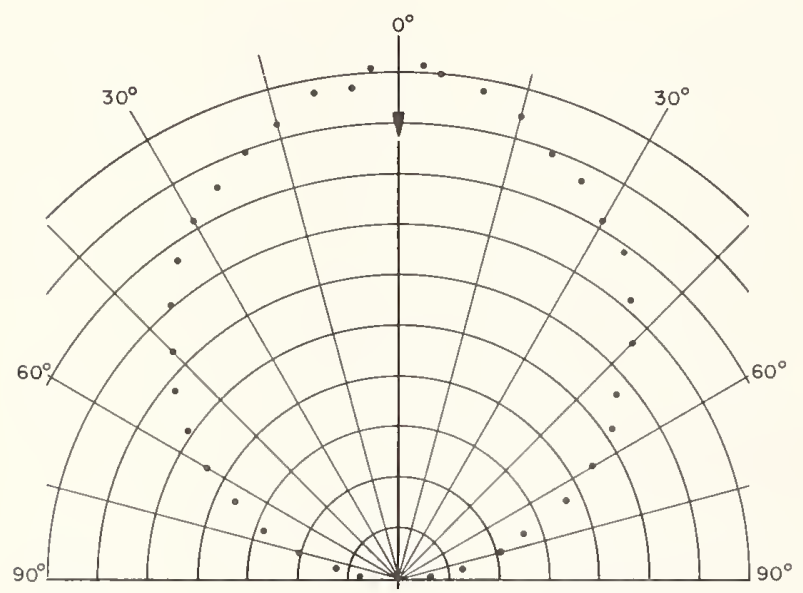

Figure A11. Directional Distribution of Reflected Flux for Sodium Chloride at $1.15 \mu \mathrm{m}$ and $\theta=0^{\circ}$

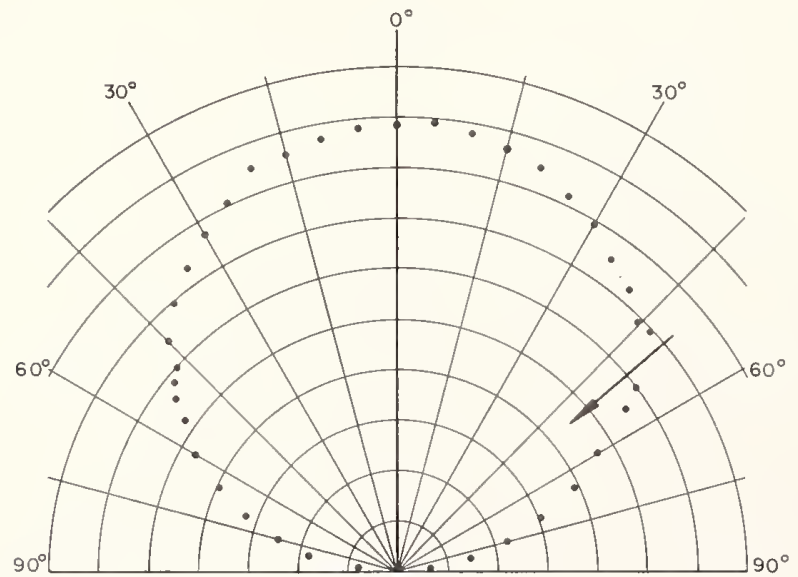

Figure A13. Directional Distribution of Reflected Flux for Sodium chloride at $1.15 \mu \mathrm{m}$ and $\theta=50^{\circ}$

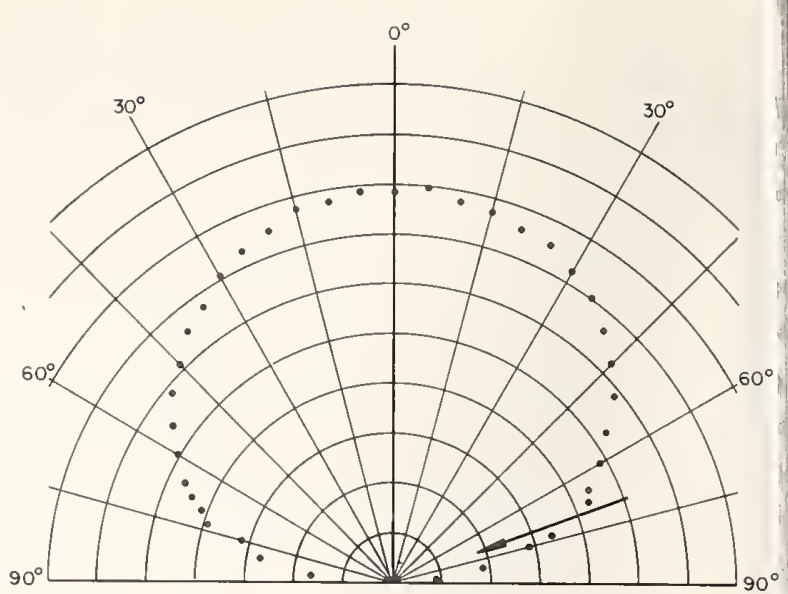

Figure A10. Directional Distribution of Reflected Flux for Sodium Chloride at $0.6328 \mu \mathrm{m}$ and $\theta=75^{\circ}$

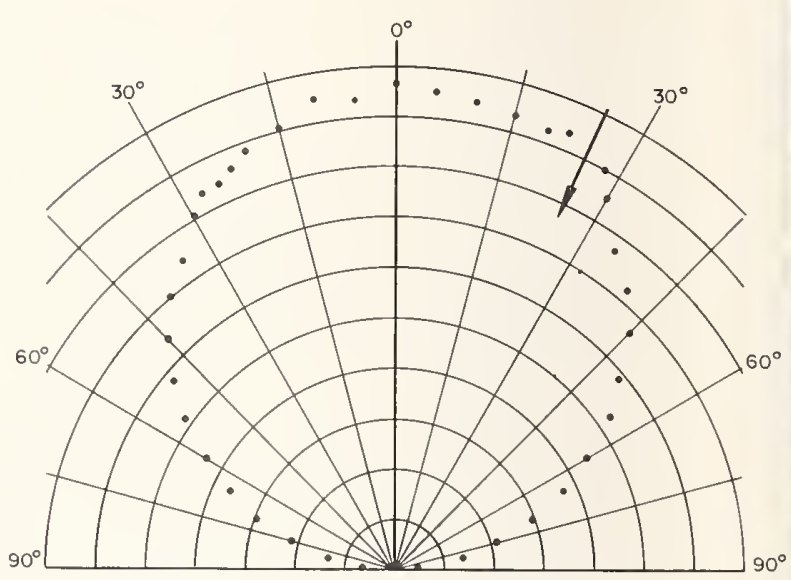

Figure A12. Directional Distribution of Reflected Flux for Sodium Chloride at $1.15 \mu \mathrm{m}$ and $\theta=25^{\circ}$

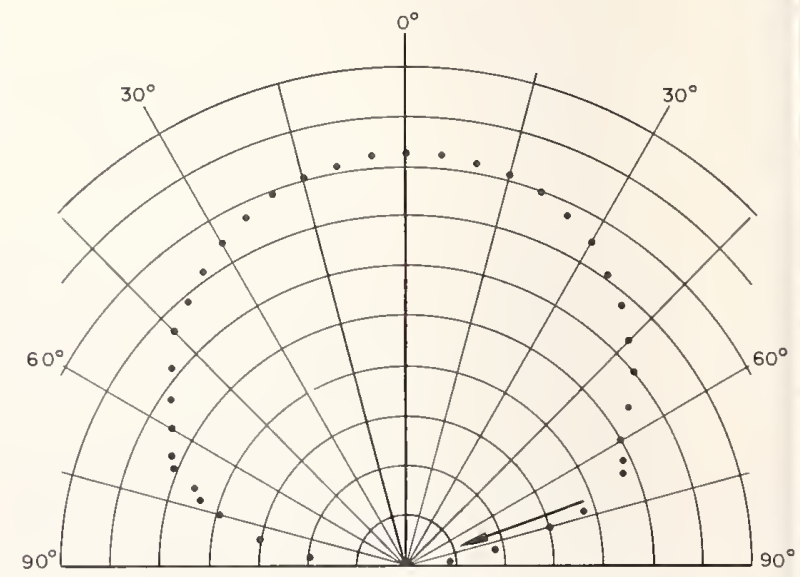

Figure A14. Direction 1 Distribution of Reflected Flux for Sodium chloride at $1.15 \mu \mathrm{m}$ and $\theta=75^{\circ}$ 
To obtain the directional distribution of flux at $3.39 \mathrm{n}$, the laser was first tried as the source and a thermopile as the detector. Since the results were not satisfactory due to the low signal-tonoise ratio, a slightly different technique was employed. As shown in Figure A15, a Globar source was mounted above and somewhat behind the sample. A spherical mirror imaged the source onto the sample with a magnification of $1: 1$. A diaphragm in front of the source restricted the haight of the image to approximately $1 / 2$ inch. To avoid errors due to flux reaching the detector by way of interreflections between different parts in the room and the sample, a chopper was mounted right in front of the spherical mirror. The signal from the thermopile was amplified in a Brower Model 129 synchronous amplifier and then displayed on an integrating digital voltmeter. To select the desired spectral band, a long wavelength pass filter was mounted between the light baffle and the detector. If it is assumed that the spectral distribution of the flux from a Globar can be approximated by that of a blackbody at $1400^{\circ} \mathrm{K}$, then the spectral distribution of flux reaching the detector can be found by multiplying the monochromatic flux at any wavelength, as given by Planck's equation, by the spectral, directionalhemispherical reflectance $7 /$ of the sample and the spectral transmittance of the filter at the same wavelength. This, of course, assumes also that the thermopile is a grey detector over the wavelength region considered. Figure Al6 shows the relative spectral composition of the flux as seen by the detector expressed as flux per unit wavelength interval, normalized to the maximum which occurs at $3.7 \mu m$. As seen from this figure, most of the flux is centered around $4 \mu \mathrm{m}$. There is no flux at wavelengths shorter than $3 \mu \mathrm{m}$, and flux at wavelengths longer than 5.9k is neglïgible. Figures A17, A18 and A19 show the directional distribution of flux of the spectral quality of Figure Al6 for angles of incidence of 0 , 30 and $60^{\circ}$. Due to the large angle subtended by the spherical mirror at the location of the sample, it was not possible to make measurements close to the direction of incidence. At large angles of incidence, the image of the source on the sample became quite large so that no measurements were made at angles of incidence greater than $60^{\circ}$. As can be seen from Figure A7 to A14, inclusive, and Figures A17, Al8 and A19, there is no narked difference in the directional distribution of flux at $0.6328 \mathrm{~m}$, $1.15 \mathrm{~m}$ and the wavelengths using the IWP filter. All in all, it can be said that the NaCl coating proved to be a very good diffuser even at high angles of incidence. In fact, comparison of data showed that the NaCl coating is at least as good a diffuser as magnesium carbonate or magnesium oxide, $i=$ not better.

\section{Iㅣ} Actually the bi-directional reflectance for the solid angles of the experiment and the particular pair of directions $\left(\theta ; \varphi ; \theta^{\prime}, \varphi^{\prime}\right)$ would have to be used. But since the interest was only in the relative spectral distribution and assuming proportionality between bi-directional and directional-hemispherical reflectance, the approach as used above is justified. 


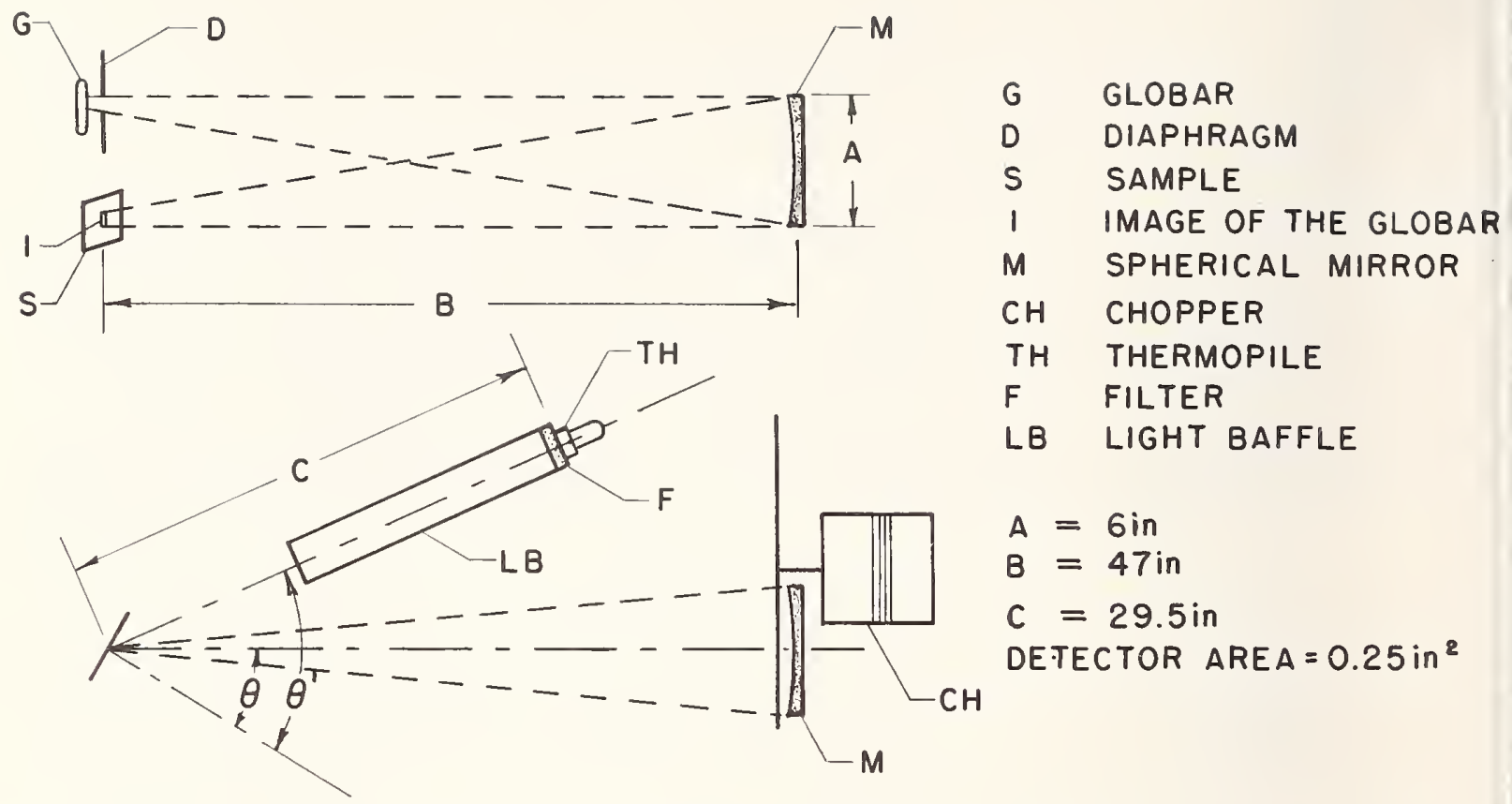

Figure A15. Globar-Source and Gonioreflectometer

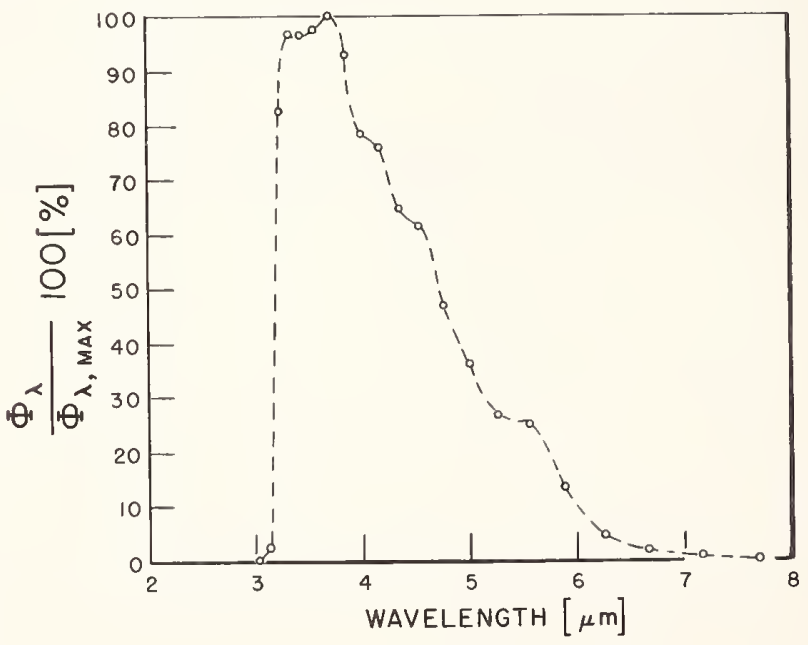

Figure A16. Spectral Distribution of Reflected Filtered Globar Flux 


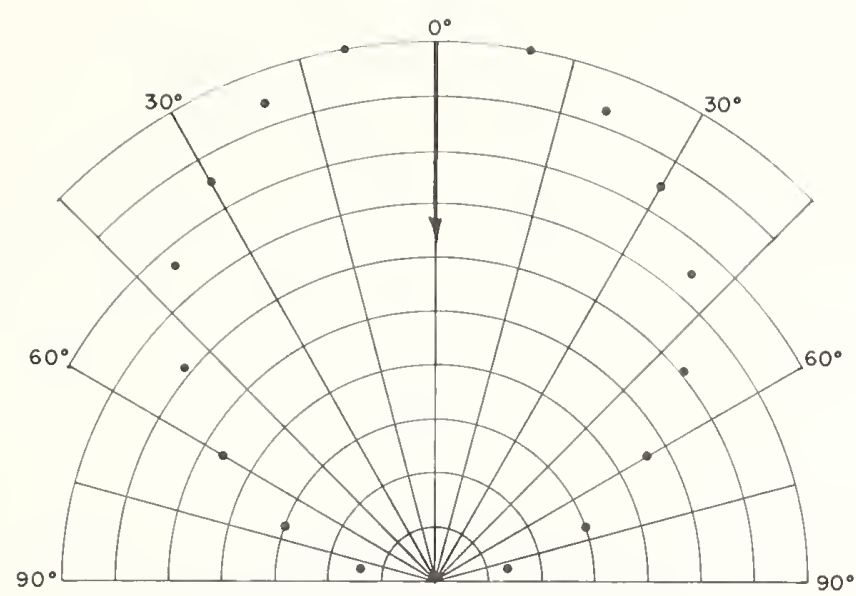

Figure A17. Directiona1 Distribution of Reflected Filtered Globar flux at $\theta=0^{\circ}$

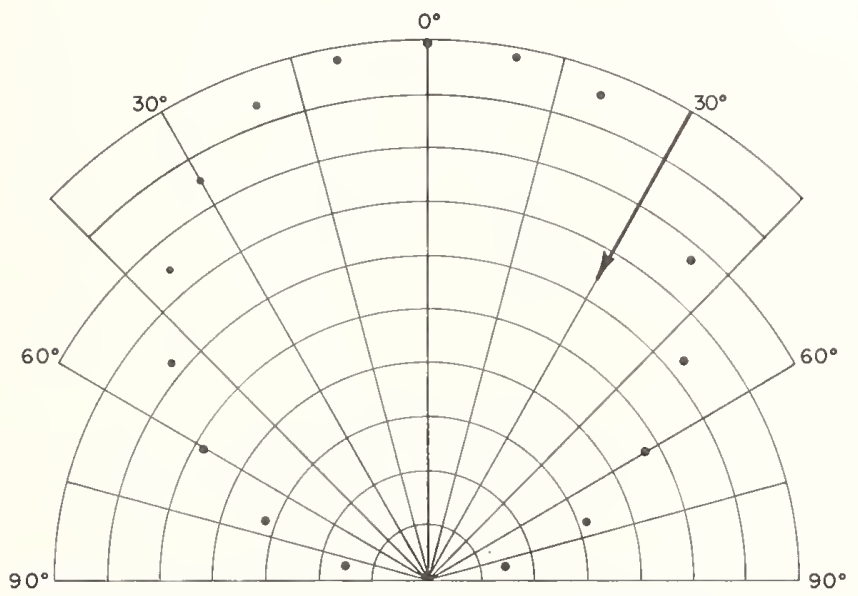

Figure A18. Directional Distribution of Reflected Filtered Globar flux at $\theta=30^{\circ}$

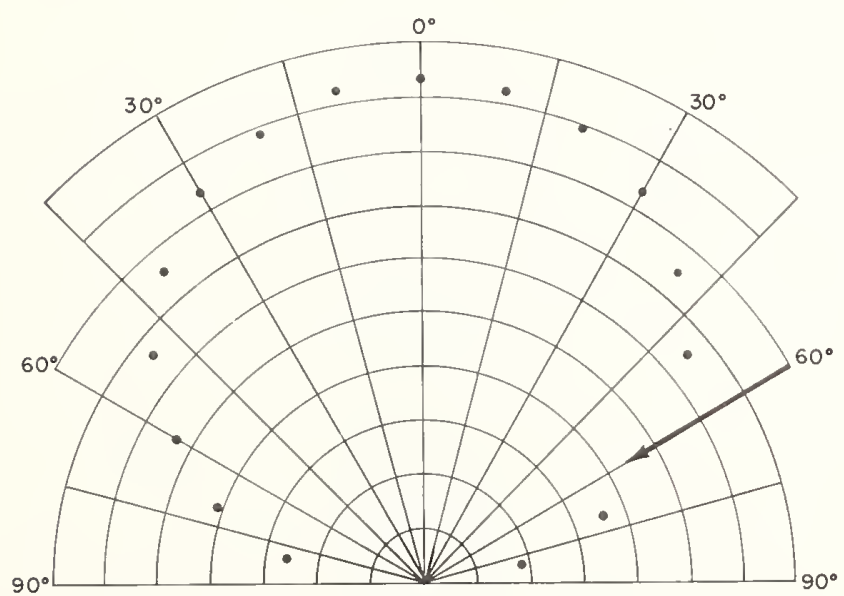

Figure A19. Directional Distribution of Reflected Filtered Globar flux at $\theta=60^{\circ}$ 


\section{Appendix B}

KIRCHHOFF'S LAW AND THE IAW OF RECIPROCITY

The integrating sphere analysed in detail in Section 3.3 measures the directional-hemispherical reflectance symbolized as $\rho(\theta, \varphi ; 2 \pi)$ or to be more specific $\rho\left(12^{\circ} ; 2 \pi\right)$. (It is assumed that the reflectance is independent of the azimuthal angle.) For many applications, for example in pyrometry, the desired quantity is the directional emittance rather than directional-hemispherical reflectance. The relationship used for or aque bodies to calculate emittance is

$$
1-p(\theta, \varphi ; 2 \pi)=\epsilon(\theta, \varphi) \text {. }
$$

In the following section, it will be shown that the use of Kirchhoff's law and the law of reciprocity are required to derive equation (B1).

Assume an isothermal enclosure. Then it can be proven [9] that the following statements are true:

a. The irradiance is independent of location and direction (isotropic radiation).

b. The monochromatic irradiance is equal to the monochromatic emissive power of a blackbody at the temperature of the enclosure.

Let the walls of the enclosure be formed by an opaque material which reflects in some diffuse manner. Using Judd's [20] definition of hemispherical-directional reflectance, $\rho(2 \pi ; \theta, \varphi)$, as ratio of flux reflected per unit area into a small solid angle d $\omega$ centered around a direction ( $\theta, \varphi$ ) to that incident over the whole hemisphere on the same area, we apply an energy balance to a small area dA of this enclosure. Since the radiation is isotropic and $\mathrm{dA}$ is in thermal equilibrium, the flux incident on $\mathrm{dA}$ within a small solid angle dw centered around a given direction $(\theta, \varphi)$ has to be equal to the flux leaving dA within the same solid angle dw. The flux leaving dA within dw consists of flux truly emitted and flux which has been reflected into $\mathrm{d} \omega$, thus

$$
\mathrm{LdA} \cos \theta \mathrm{d} \omega=\epsilon(\theta, \varphi) \mathrm{I}_{\mathrm{b}} \mathrm{dA} \cos \theta \mathrm{d} \omega+\rho(2 \pi ; \theta, \varphi) \quad \pi \mathrm{L} \mathrm{dA} .
$$

The two part notation $(2 \pi ; \theta, \varphi)$ means that the body is irradiated over the total hemisphere (uniform irradiance is always assumed) and the small solid angle of collection dw is centered around the direction $(\theta, \varphi)$.

From statement b, it follows that the monochromatic radiance inside the enclosure is equal to the monochromatic radiance of a blackbody at the same temperature. Therefore $L=E_{b}$ and equation (B2) becomes

or

$$
1=\epsilon(\theta, \varphi)+\pi \rho(2 \pi ; \theta, \varphi) / \cos \theta d \omega
$$

$$
\epsilon(\theta, \varphi)=1-\frac{\pi \rho(2 \pi ; \theta, \varphi)}{\cos \theta d \omega}
$$

where $\epsilon(\theta, \varphi)$ is the directional emittance and is defined as the ratio of the radiance of a body in the direction $(\theta, \varphi)$ due to emitted flux only, to the radiance of a blackbody. (For a blackbody, or perfect radiator as it is sometimes called, the radiance is independent of direction; and therefore, no direction needs to be specified.)

When we compare equation (B3) with equation (B1), we see that in order for equation (B1) to be true it has to be shown that:

$$
\rho(\theta, \varphi ; 2 \pi)=\frac{\pi \rho(2 \pi ; \theta, \varphi)}{\cos \theta d \omega}
$$


In words: if a surface area dA is irradiated within a solid angle dw around the direction ( $\theta, \varphi)$ with a radiance $L$, then the flux reflected into the entire hemisphere above dA has to be equal to the flux which is reflected into the same solid angle dw around the same direction $(\theta, \varphi)$ when dA is uniformly irradiated over the whole hemisphere with the same radiance $L$.

Before the validity of equation (B4) can be proven, it is necessary to go one step back and discuss the reciprocity for a more fundamental quantity. As shown in Figure B1, assume that a differential surface area dA is irradiated by a source with an area dA from a direction $\left(\theta_{1}, \varphi_{1}\right)$. The source area $\mathrm{dA}_{1}$ is perpendicular to the direction $\left(\theta_{1}, \varphi_{1}\right)$, is located at a distance $r_{1}$ from dA and is radiating with the radiance $\mathrm{L}$. The flux incident on $\mathrm{dA}$ is then

$$
I \frac{d A_{1}}{r_{1}{ }^{2}} d A \cos \theta_{1} \text {. }
$$

This flux is reflected by dA in some manner which results in a radiance $L^{\prime}$ in the direction ( $\theta_{2}, \varphi_{2}$ ). Let a detector with a receiving area $\mathrm{dA}_{2}$ be positioned in the direction $\left(\theta_{2}, \varphi_{2}\right)$ at a distance $r_{2}$ from $\mathrm{dA}$ and perpendicular to the direction $\left(\theta_{2}, \varphi_{2}\right)$. The flux received by the detector becomes

$$
L^{\prime} \frac{\mathrm{dA}_{2}}{\mathrm{r}_{2}{ }^{2}} \mathrm{dA} \cos \theta_{2} \text {. }
$$

Using Judd's definition of bi-directional reflectance $\rho\left(\theta_{1}, \ell_{1} ; \theta_{2}, \varphi_{2}\right)$ such that

$$
\begin{aligned}
& \rho\left(\theta_{1}, \varphi_{1} ; \theta_{2}, \varphi_{2}\right)=\frac{L^{\prime}\left(\theta_{2}, \varphi_{2}\right) \cos \theta_{2} d \omega_{2}}{L\left(\theta_{1}, \varphi_{1}\right) \cos \theta_{1} d_{1}}, \\
& d \omega_{1}=\frac{d A_{1}}{r_{1}^{2}} \text { and } d \omega_{2}=\frac{\mathrm{dA}_{2}}{r_{2}^{2}},
\end{aligned}
$$

where

the quantity of flux $\mathrm{d} \Phi$, received by the detector in this first case becomes

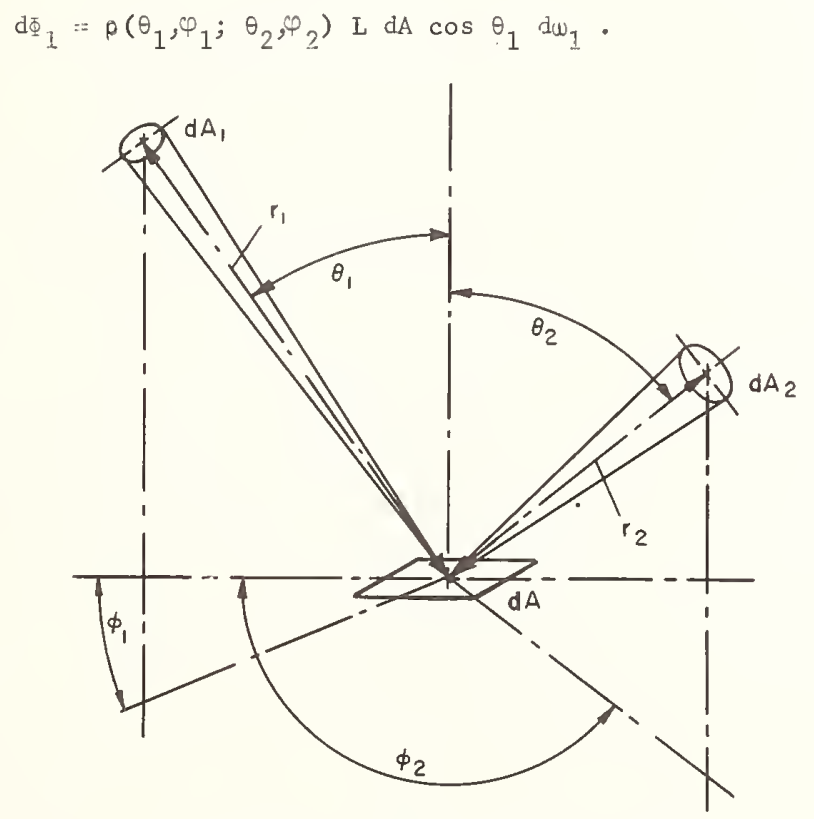

Figure B1. Bi-directional Geometry 
In the second case, the roles of $\mathrm{dA}_{1}$ and $\mathrm{dA}_{2}$ are interchanged. Now let $\mathrm{dA}_{2}$ be the source with a radiance $\mathrm{L}$ and let $\mathrm{dA}_{1}$ become the detector area. The flux received by the detector is then

$$
\mathrm{d} \Phi_{2}=\rho\left(\theta_{2}, \varphi_{2} ; \theta_{1}, \varphi_{1}\right) \text { LdA } \cos \theta_{2} \mathrm{~d} \omega_{2} .
$$

According to the reciprocity theorem by Sommerfeld-Pfrang [22], $\mathrm{d}_{1}{ }_{1}$, has to be equal to $\mathrm{d} \Phi_{2}$, and from equations (B6) and (B7) it then follows that

$$
\frac{\rho\left(\theta_{1}, \varphi_{1} ; \theta_{2}, \varphi_{2}\right)}{\cos \theta_{2} d \omega_{2}}=\frac{\rho\left(\theta_{2}, \varphi_{2} ; \theta_{1}, \varphi_{1}\right)}{\cos \theta_{1} d \omega_{1}}
$$

In order for reciprocity to hold, it was necessary to leave $\mathrm{dA}_{1}$ and $\mathrm{dA}_{2}$ in place when source and receiver were interchanged. In other words, in the first sase the source had an emitting area dA 1 and irradiated area $\mathrm{dA}$ from the direction $\left(\theta_{1}, \varphi_{1}\right)$ and the detector with a receiver area $\mathrm{dA}_{2}$ received $\mathrm{flux}$ reflected into the direction $\left(\theta_{2} \varphi_{2}\right)$, while in the second case the source with an emitting area dA 2 irradiated area dA from the direction $\left(\theta_{2}, \varphi_{2}\right)$ and the detector with a receiving area dA intercepted the flux reflected into the direction $\left(\theta_{1}, \varphi_{1}\right)$. Therefore if source and detector subtend different solid angles at $\mathrm{dA}$ and their respective positions are interchanged, then it is also necessary to interchange their solid angles. A more detailed discussion of this point is given by Fragstein [22].

In the first case when we integrate $\mathrm{d}_{1}$, the reflected flux, with respect to d $\omega_{2}$ over the hemispher we get:

$$
\Phi_{1}=\int_{2 \pi} \mathrm{d} \Phi_{1}=\int_{2 \pi} L^{\prime} \mathrm{dA} \cos \theta_{2} \mathrm{~d} \omega_{2}
$$

which by means of eq. (B5) becomes

$$
\bar{\Phi}_{1}=L d A \cos \theta_{1} d \omega_{1} \int_{2 \pi} \rho\left(\theta_{1}, \varphi_{1} ; \theta_{2}, \varphi_{2}\right)
$$

which represents all of the reflected flux.

The directional-hemispherical reflectance is by definition

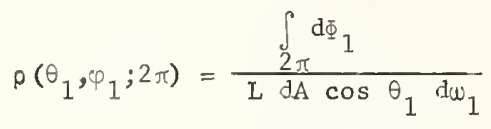

which by using equation (B9) becones

$$
\rho\left(\theta_{1}, \varphi_{1} ; 2 \pi\right)=\int_{2 \pi} \rho\left(\theta_{1}, \varphi_{1} ; \theta_{2}, \varphi_{2}\right) .
$$

In the second case $\mathrm{dA}_{2}$ is irradiating $\mathrm{dA}$ from the direction $\left(\theta_{2}, \varphi_{2}\right)$ and the flux received by $\mathrm{dA}_{1}$ was expressed in eq. (B7) as

$$
\mathrm{d}_{2}=\rho\left(\theta_{2} \rho \rho_{2} ; \theta_{1} \rho \rho_{1}\right) \mathrm{L} \mathrm{dA} \cos \theta_{2} \mathrm{~d} \omega_{2} \cdot
$$


To find the flux received by $\mathrm{dA}_{2}$ when $\mathrm{dA}$ is irradiated uniformly over the hemisphere with a radiance $\mathrm{L}$, we integrate (B7) with respect to $\mathrm{d}_{2}$ and get

$$
\Phi_{2}=\operatorname{LdA} \int_{2 \pi} \rho\left(\theta_{2}, \varphi_{2} ; \theta_{1}, \varphi_{1}\right) \cos \theta_{2} \mathrm{~d} \omega_{2}
$$

Using eq. (B8), eq. (B11) can be written as

$$
\Phi_{2}=\mathrm{LdA} \cos \theta_{1} \mathrm{~d} \omega_{1} \int_{2 \pi} \rho\left(\theta_{1}, \varphi_{1} ; \theta_{2}, \varphi_{2}\right) .
$$

The total flux incident on $\mathrm{dA}$ is $\pi \mathrm{LdA}$ and the hemispherical-directional reflectance can be written as

$$
\begin{aligned}
\rho\left(2 \pi ; \theta_{1}, \varphi_{1}\right) & =\frac{\Phi_{2}}{\pi \mathrm{Ld} A} \\
& =\frac{1}{\pi} \cos \theta_{1} \mathrm{~d}_{1} \int_{2 \pi} \rho\left(\theta_{1}, \varphi_{1} ; \theta_{2}, \varphi_{2}\right)
\end{aligned}
$$

Comparing eq. (B10) and (B13) we find that

$$
\frac{\pi \rho\left(2 \pi ; \theta_{1}, \rho_{1}\right)}{\cos \theta_{1} \mathrm{~d} \omega_{1}}=\rho\left(\theta_{1}, \varphi_{1} ; 2 \pi\right)
$$

which shows that eq. (B4) holds which in turn proves the validity of eq. (B1), namely

$$
\epsilon(\theta, \varphi)=1-\rho(\theta, \varphi ; 2 \pi) .
$$

The above equation was derived under the condition that dA is in thermodynamic equilibrium with its surroundings. But since the emittance and reflectance of a specimen of a given material depend only on its surface condition and temperature, the equation can also be applied to nonequilibrium situations.

In the light of the importance of bi-directional reflectance measurements to adequately describe the reflective properties of materials it seems useful to discuss in more detail its concept. Since we can only measure a finite quantity of flux, we never mean truly a single direction when we talk about directional irradiance or directional exitance. But this immediately raises the question of how small the solid angles have to be to adequately approximate a single direction. In the case of directional irradiance, the condition has been defined by McNicholas [1] as follows:

The solid angular extent $\mathrm{d}_{\boldsymbol{\prime}}{ }^{\prime}$ of the incident flux shall be so small that a further decrease will not alter measurably either the angular distribution of the reflected flux or its ratio to the incident flux.

In the case of directional exitance, an analogous condition would be:

The solid angular extent dw' of the measured reflected flux shall be so small that a further decrease will not alter measurably the ratio of the reflected flux (or its electrical analog) to the acceptance solid angle, $\mathrm{d} \omega^{\prime}$. The bi-directional reflectance is defined as

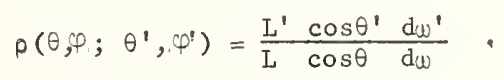


Assuming that the conditions defined above have been met, then we can see the following. If we change $L$, the radiance of the incident radiation, then $L^{\prime}$, the radiance of the exitent radiation will be altered proportionally. The same is true of the solid angle dw. For example if we cut the solid angle of the incilent radiation in half, then $L^{\prime}$ will drop to half of its original value. In other words $\rho\left(\theta, \varphi ; \theta^{\prime}, \varphi^{\prime}\right)$ is invariant with respect to the solid angle dw and the incilent radiance L. Or the other hand, we are free to choose any size of the solid angle dw' within the limit imposed by the condition of directional exitance. This means that we can obtain values ranging from 0 to a maximum value depending on the size of the acceptance solid angle of the instrument used. .

At first glance, it might seem that a more fundamental quantity would be the radiance ratio defined as

$$
\frac{L^{\prime}\left(\theta^{\prime}, \varphi^{\prime}\right)}{L(\theta, \varphi)} .
$$

But from the forgoing discussion of bi-directional reflectance, we see that while the radiance ratio is invariant with respect to $\mathrm{d} \omega^{\prime}$ and $\mathrm{L}$, the radiance of the reflected flux $\mathrm{L}^{\prime}$ is directly proportional to $\mathrm{d} w$, which again can be varied freely within the limits given by the condition for directional irradiance. Therefore, in addition to the radiance ratio, this time one needs to know the solid angle of the incident flux to reduce it to a useful quantity.

So we see that the bi-directional reflectance and the radiance ratio are not only functions of the material and its surface condition but depend also to a large extent on the instrument used for the measurements. This, for example, would make it impossible to compare directly bi-directional reflectance or radiance ratio data obtained by two different instruments using different solid angles.

In eq. (B8) it has been shown that reciprocity holds for the quantity

$$
\frac{\rho\left(\theta, \varphi ; \theta^{\prime}, \varphi^{\prime}\right)}{\cos \theta^{\prime} \mathrm{d} \omega^{\prime}}
$$

To understand the physical significance of this quantity we use eq. (B14) which defines bi-directional reflectance and obtain

$$
\frac{\theta\left(\theta, \varphi ; \theta^{\prime}, \varphi^{\prime}\right)}{\cos \theta^{\prime} d \omega^{\prime}}=\frac{L^{\prime}\left(\theta^{\prime}, \varphi^{\prime}\right)}{L(\theta, \varphi) \cos \theta d \omega} .
$$

Now we see that this quantity can be interpreted as the radiance in the direction ( $\theta$ !, $\varphi$ :) per unit irradiance from the direction $(\theta, \varphi)$.

From the discussion of bi-directional reflectance, we have seen that the radiance $L^{\prime}$ changes proportionally with any change of the solid angle dw as well as the incident radiance L. The dependence on the solid aingle d $\omega^{\prime}$ has been rencved. Therefore the quotient $L^{\prime}\left(\theta^{\prime}, \varphi^{\prime}\right) /(L \cos \theta$ d $\omega)$ which ve give tile symbol $\ell_{r}\left(\theta, 0 ; 9^{\prime}, \varphi^{\prime}\right)$ and call the relative radiance, does not depend on the instrument used and is only a function of the sample material and its surface condition.

The importance of the relative radiance has been widely recognized and the quantity has been used in the literature under the names of partial reflectance [23], reflection distribution function [18] and others. 
$\mathrm{L}(\theta, \varphi)$

$I^{\prime}{ }^{\prime}\left(\theta^{\prime}, \varphi^{\prime}\right)$

$\mathrm{L}_{\mathrm{b}}$

Surface Properties

$6(\theta, \varphi)$

$\rho(\theta, \varphi ; 2 \pi)$

$p\left(\theta, \varphi ; \theta^{\prime}, \varphi^{\prime}\right)$

$\frac{L^{\prime}\left(\theta^{\prime}, \varphi^{\prime}\right)}{L(\theta, \varphi)}$

$l_{r}\left(\theta, \varphi ; \theta^{\prime}, \varphi^{\prime}\right)$
Radiance in the direction $(\theta, \varphi)$.

Radiance due to flux reflected in the direction $\left(\theta^{\prime}, \varphi^{\prime}\right)$.

Radiance of a blackbody (perfect radiator).

Directional emittance for the direction $(\theta, \varphi)$. Ratio of the radiance of a body in the direction $(\theta, \varphi)$ due to emitted flux only, to the radiance of a blackbody at the same temperature.

Directional-hemispherical reflectance. Ratio of flux reflected in all directions to the flux incident within a small solid angle centered around the direction $(\theta, \varphi)$.

Bi-directional reflectance. Ratio of flux reflected into a small solid angle $d \omega^{\prime}$ centered around the direction $\left(\theta^{\prime}, \varphi^{\prime}\right)$ to the flux incident within a small solid angle $d \omega$ centered around the direction $(\theta, \varphi)$.

Radiance ratio. Ratio of radiance due to reflected flux in the direction $\left(\theta^{\prime}, \varphi^{\prime}\right)$ to the radiance of the incident flux in the direction $(\theta, \varphi)$. Relative radiance. Ratio of radiance due to reflected flux in the direction $\left(\theta^{*}, \varphi^{\prime}\right)$ to the flux incident per unit area within 2 small solid angle $d \omega$ crntered around the direction $(\theta, \varphi)$, or: radiance in the direction $\left(\theta^{\prime}, \varphi^{\prime}\right)$ per unit Irradiance from the direction $(\theta, \varphi)$. 

PERIODICALS

JOURNAL OF RESEARCH reports $\mathrm{Na}$ tional Bureau of Standards research and development in physics, mathematics, chemistry, and engineering. Comprehensive scientific papers give complete details of the work, including laboratory data, experimental procedures, and theoretical and mathematical analyses. Illustrated with photographs, drawings, and charts.

\section{Published in three sections, available separately:}

\section{- Physics and Chemistry}

Papers of interest primarily to scientists working in these fields. This section covers a broad range of physical and chemical research, with major emphasis on standards of physical measurement, fundamental constants, and properties of matter. Issued six times a year. Annual subscription: Domestic, \$5.00; foreign, $\$ 6.00^{*}$.

\section{- Mathematics and Mathematical Physics}

Studies and compilations designed mainly for the mathematician and theoretical physicist. Topics in mathematical statistics, theory of experiment design, numerical analysis, theoretical physics and chemistry, logical design and programming of computers and computer systems. Short numerical tables. Issued quarterly. Annual subscription: Domestic, $\$ 2.25$; foreign, $\$ 2.75 *$.

\section{- Engineering and Instrumentation}

Reporting results of interest chiefly to the engineer and the applied scientist. This section includes many of the new developments in instrumentation resulting from the Bureau's work in physical measurement, data processing, and development of test methods. It will also cover some of the work in acoustics, applied mechanics, building research, and cryogenic engineering. Issued quarterly. Annual subscription: Domestic, $\$ 2.75$; foreign, $\$ 3.50 *$.

\section{TECHNICAL NEWS BULLETIN}

The best single source of information concerning the Bureau's research, developmental, cooperative and publication activities, this monthly publication is designed for the industry-oriented individual whose daily work involves intimate contact with science and technology-for engineers, chemists, physicists, research managers, product-development managers, and company executives. Annual subscription: Domestic, \$1.50; foreign, $\$ 2.25^{*}$.

- Difference in price is due to extra cost of foreign mailing.
NONPERIODICALS

Applied Mathematics Series. tables, manuals, and studies.

Mathematical

Building Science Series. Research results, test methods, and performance criteria of building materials, components, systems, and structures.

Handbooks. Recommended codes of engineering and industrial practice (including safety codes) developed in cooperation with interested industries, professional organizations, and regulatory bodies.

Miscellaneous Publications. Charts, administrative pamphlets, Annual reports of the Bureau, conference reports, bibliographies, etc.

Monographs. Major contributions to the technical literature on various subjects related to the Bureau's scientific and technical activities.

National Standard Reference Data Series. NSRDS provides quantitative data on the physical and chemical properties of materials, compiled from the world's literature and critically evaluated.

Product Standards. Provide requirements for sizes, types, quality and methods for testing various industrial products. These standards are developed cooperatively with interested Government and industry groups and provide the basis for common understanding of product characteristics for both buyers and sellers. Their use is voluntary.

Technical Notes. This series consists of communications and reports (covering both other agency and NBS-sponsored work) of limited or transitory interest.

\section{CLEARINGHOUSE}

The Clearinghouse for Federal Scientific and Technical Information, operated by NBS, supplies unclassified information related to Governmentgenerated science and technology in defense, space, atomic energy, and other national programs. For further information on Clearinghouse services, write:

Clearinghouse

U.S. Department of Commerce Springfield, Virginia 22151

Order NBS publications from:

Superintendent of Documents

Government Printing Office

Washington, D.C. 20402 


\section{U.S. DEPARTMENT OF COMMERCE}

WASHINGTON, D.C. 20230

OFFICIAL BUSINESS 